\title{
直接三氟甲硫基化试剂及方法的研究进展
}

\author{
张盼盼吕龙沈其龙* \\ (有机氟化学重点实验室 中国科学院上海有机化学研究所 中国科学院大学 上海 200032)
}

\begin{abstract}
摘要 三氟甲硫基脂溶性高、吸电子能力强, 将其引入药物分子中可以改变分子药代动力学以及物理化学特性, 提高 代谢稳定性. 含三氟甲硫基的分子在医药、农药、材料以及含氟日用品等领域得到了广泛的应用. 因此, 如何高效地向 分子中引入三氟甲硫基团成为了近些年来有机氟化学领域的一个研究热点. 经过几年的研究, 新的三氟甲硫基化试剂 及方法得到了快速的发展, 为药物化学家提供了方便简洁地引入三氟甲硫基团的工具箱. 在本篇综述中, 将首先简要 介绍间接引入三氟甲硫基团的方法，随后将对直接引入三氟甲硫基团的方法，尤其是近些年来发展的过渡金属催化、 亲电三氟甲硫基化以及自由基类型的三氟甲硫基化方法做着重的介绍. 最后将对三氟甲硫基化领域存在的问题和难点 进行简要的展望.
\end{abstract}

关键词 三氟甲硫基化; 有机氟化学; 间接法; 直接法

\section{Recent Progress on Direct Trifluoromethylthiolating Reagents and Methods}

\author{
Zhang, Panpan Lu, Long Shen, Qilong* \\ (Key Laboratory of Organofluorine Chemistry, Shanghai Institute of Organic Chemistry, University of Chinese Academy of \\ Sciences, Chinese Academy of Sciences, Shanghai 200032)
}

\begin{abstract}
With a significantly high Hansch's hydrophobicity parameter $(\pi=1.44)$, electron-withdrawing trifluoromethylthio group $\left(\mathrm{CF}_{3} \mathrm{~S}-\right)$ has been considered as one of the most lipophilic substituents and privileged fragments that are able to improve drug molecules' pharmacokinetic and physicochemical properties such as lipophilicity and metabolic stability. It is well-known that incorporation of the trifluoromethylthio group into small molecules greatly enhances its ability to cross lipid membranes and in vivo absorpotion rate. In addition, the high electronegativity of the trifluoromethylthio group significantly improves the small molecule's stablity in acidic environments. Not surprisingly, the trifluoromethylthio group has been of special attention not only from the academia but also from pharmaceutical and agrochemical industry for their use in isostere-based drug design. Development of highly efficient methods for the introduction of the trifluoromethylthio group into small molecules, thereafter, has become a subject of recent focus in the field of organic chemistry. In the early 1960s, a few methods for the formation of trifluoromethylthioethers were reported, which typically involved halogen exchange of the trichloromethyl-substituted compounds and trifluoromethylation of thiolated substrates. However, the conditions of these methods were harsh and incompatible with many common functional groups. Since 2008, new reagents and methods that were able to efficiently incorporate the trifluoromethylthio group under mild conditions have emerged, that pave the way for the facile introduction of trifluoromethylthio group into site-specific positions of the target molecules. In this review, we will first briefly introduce the indirect strategies for trifluoromethylthiolation including halogen exchange and trifluoromethylation of thiolated substrates, and then focus on the direct trifluoromethylthiolation strategies including the transition metal-catalyzed trifluoromethylthiolation reactions, electrophilic trifluoromethylthiolation reactions with electrophilic trifluoromethylthiolating reagents and radical trifluoromethylthiolations. These methods represent the most straightforward and promising approaches for the incorporation of the trifluoromethylthio group into small molecules. At the end, we will discuss the remaining problems and challenges in this particular field.
\end{abstract}

Keywords fluorine chemistry; trifluoromethylthiolation; indirect strategy; direct method

\section{1 引言}

2016 年 10 月, 中国科学院科技战略咨询研究院、 中国科学院文献情报中心与 Clarivate Analytics 公司(原
汤森路透知识产权与科技事业部)联合发布了《2016 研 究前沿》报告 ${ }^{[1]}$. 从该报告的统计数据中我们发现, 在化 学与材料科学领域中, 有关三氟甲硫基化反应的研究位 列第二!中国科学家在该研究领域发表核心论文多达 47

\footnotetext{
* E-mail: shenql@mail.sioc.ac.cn

Received May 9, 2017; published June 20, 2017.
}

Project supported by the National Natural Science Foundation of China (Nos. 21625206, 21632009, 21372247, 21572258, 21572259, 21421002) and the Strategic Priority Research Program of the Chinese Academy of Sciences (No. XDB20000000).

项目受国家自然科学基金(Nos.21625206，21632009，21372247，21572258，21572259，21421002)和中国科学院战略性先导科技专项(B 类) (No. XDB20000000)资助. 
篇, 被引频次达到了 3168 次之多! 由此可见, 对于三氟 甲硫基化反应的研究是目前有机化学领域的一个热点.

表 12016 年化学与材料科学领域热点前沿

Table 1 Frontiers of chemistry and material science in 2016

\begin{tabular}{cccc}
\hline 排名 & 热点前沿 & 核心论文 & 被引频次 \\
\hline 1 & 基于非富勒烯受体的有机太阳能电池 & 41 & 2249 \\
2 & 三氟甲硫基化反应 & $\mathbf{4 7}$ & $\mathbf{3 1 6 8}$ \\
3 & 摩擦纳米发电机 & 43 & 2846 \\
4 & 非贵金属电解水纳米催化剂 & 26 & 2427 \\
5 & 金催化的有机合成 & 23 & 2062 \\
6 & 高效钻钠矿型太阳能电池 & 30 & 1647 \\
7 & 半导体/石墨烯纳米复合物光催化剂 & 21 & 3176 \\
8 & 白光 LED 用苂光粉 & 44 & 4690 \\
9 & 石墨烯过滤膜 & 22 & 3125 \\
10 & 锂离子电池 & 4 & 1998 \\
\hline
\end{tabular}

摘自《2016 研究前沿》.

该领域受到研究者们的广泛关注, 其原因在于三氟 甲硫基独特的性质以及其重大的应用前景. 三氟甲硫基 官能团有着较强的吸电子能力, 可以有效地降低底物的 电子云密度, 从而提高分子的代谢稳定性; 硫原子有着 多种氧化态, 可以进一步氧化成亚砜以及砜基官能团, 从而可以更加灵活地通过电性来对底物的活性进行调 控; 脂溶性对生物体对药物分子的吸收、分布以及对药 物分子到达靶点的能力有着决定性的影响. 而三氟甲硫 基官能团在常见的含氟取代基中拥有最高的 $\pi$ 系数 $(1.44)^{[2]}$, 将其引入分子中可以极大程度地提高分子的 脂溶性，增加细胞膜的穿透性.

如何向分子中引入三氟甲硫基官能团在近些年来 得到了长足和快速的发展. 关于三氟甲硫基官能团的引 入, 相关的总结也有不少 ${ }^{[3]}$, 但考虑到该领域快速的发 展, 我们认为仍然需要对该领域进行一个归纳.

\section{2 三氟甲硫基的引入}

根据底物和三氟甲硫基来源的不同，我们可以将引 入三氟甲硫基的方法分为直接法和间接法. 在该领域早 期发展的方法大部分都属于间接法, 包括含卤前体的氟 卤交换法和含硫底物的亲电、亲核以及自由基类型的三 氟甲基化方法(Scheme 1).

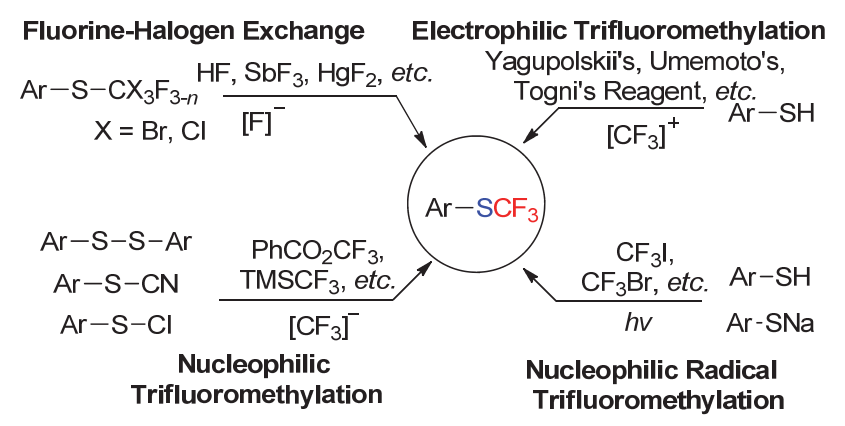

图式 1 间接引入三氟甲硫基的方法

Scheme 1 Indirect strategies for trifluoromethylthiolation
对于氟卤交换法 ${ }^{[4]}$ 而言，首先需要预先制备含卤底 物. 其次需要使用高活性的亲核性氟源如 $\mathrm{HF} 、 \mathrm{SbF}_{3}$ 等 进行氟卤交换反应. 该方法在实验室操作较为繁琐, 但 在工业生产中应用较广。

对于含硫底物的三氟甲基化方法而言, 首先也需要 预先制备含硫底物, 如二硫醚、硫氧基底物、硫氯底物 或者硫醇、硫酚类底物等. 其次采用亲核性 ${ }^{[5]}$ 、亲电性 三氟甲基源 ${ }^{[6]}$ 或者在紫外光照的条件下 ${ }^{[7]}$ 对含硫底物进 行三氟甲基化. 该方法对于简单底物比较适用，但对于 一些复杂的底物分子而言, 制备含硫底物的难度是比较 大的，也就限制了该方法在分子合成设计后期的应用.

而直接向分子中引入三氟甲硫基有着简单、高效的 优势，更有利于在分子合成设计的后期来对底物进行三 氟甲硫基化修饰. 根据三氟甲硫基的来源，我们可以将 直接三氟甲硫基化法分为亲核型、亲电型以及自由基型 三氟甲硫基化法(Scheme 2).

\section{Nucleophilic Trifluoromethylthiolation

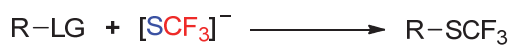

\section{Electrophilic Trifluoromethylthiolation}

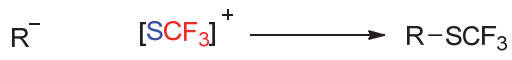

Radical Trifluoromethylthiolation

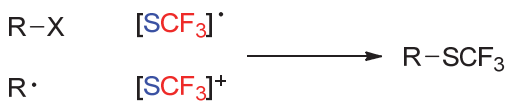

图式 2 直接引入三氟甲硫基的方法

Scheme 2 Direct strategies for trifluoromethylthiolation

\section{1 亲核三氟甲硫基化法}

\section{1 .1 亲核三氟甲硫基源}

亲核三氟甲硫基化法是采用亲核性三氟甲硫基源 与亲电底物反应来实现的. 而常用的亲核三氟甲硫基源 包括三氟甲硫基金属或非金属试剂.

Muetterties 等 ${ }^{[8]}$ 最早通过 $\mathrm{HgF}_{2}$ 和 $\mathrm{CS}_{2}$ 在 $250{ }^{\circ} \mathrm{C}$ 下制 备了首个亲核三氟甲硫基源 $\mathrm{Hg}\left(\mathrm{SCF}_{3}\right)_{2}$. 而制备的 $\mathrm{Hg}\left(\mathrm{SCF}_{3}\right)_{2}$ 可以与 $\mathrm{Cu}$ 粉或者 $\mathrm{AgNO}_{3}$ 反应来进一步制备 $\mathrm{CuSCF}_{3}$ 与 $\mathrm{AgSCF}_{3}$, 但在当时其制备的纯度不高. 随后 MacDuffie 等 ${ }^{[9]}$ 采用与 Muetterties 类似的方法, 通过 $\mathrm{AgF}$ 和 $\mathrm{CS}_{2}$ 在 $140{ }^{\circ} \mathrm{C}$ 下反应，以 $70 \% \sim 80 \%$ 的收率制备 $\mathrm{AgSCF}_{3}$. Yagupolskii 小组 ${ }^{[10]}$ 从 $\mathrm{AgSCF}_{3}$ 出发, 与 $\mathrm{CuBr}$ 发生一步复分解反应, 可以以当量的收率获得 $\mathrm{CuSCF}_{3}$ (Scheme 3).

$$
\begin{gathered}
3 \mathrm{HgF}_{2}+2 \mathrm{CS}_{2} \underset{250{ }^{\circ} \mathrm{C}}{\longrightarrow} 2 \mathrm{HgS}+\mathrm{Hg}\left(\mathrm{SCF}_{3}\right)_{2} 75 \% \\
3 \mathrm{AgF}+\mathrm{CS}_{2} \underset{140{ }^{\circ} \mathrm{C}}{\longrightarrow} \mathrm{Ag}_{2} \mathrm{~S}+\mathrm{AgSCF}_{3} 70 \% \sim 80 \% \\
\mathrm{AgSCF}_{3}+\mathrm{CuBr} \longrightarrow \mathrm{AgBr}+\mathrm{CuSCF}_{3}>99 \%
\end{gathered}
$$

图式 3 三氟甲硫录、三氟甲硫银和三氟甲硫铜的制备 Scheme 3 Preparation of $\mathrm{Hg}\left(\mathrm{SCF}_{3}\right)_{2}, \mathrm{AgSCF}_{3}$ and $\mathrm{CuSCF}_{3}$ 
随后，其他一系列的三氟甲硫基物种 $\left(\mathrm{K}, \mathrm{Cs}, \mathrm{NMe}_{4}\right.$, $\left.\mathrm{S}\left(\mathrm{NMe}_{2}\right)_{3}, \mathrm{TDAE}\right)$ 陆续被报道, Yagupolskii 小组 ${ }^{[11]}$ 采用 一锅法, 从硫粉和 Ruppert-Prakash 试剂出发, 可以简单 便捷地合成相应的 $\mathrm{Me}_{4} \mathrm{NSCF}_{3} 、 \mathrm{CsSCF}_{3}$ 等亲核性三氟甲 硫基源(Scheme 4).

$$
\mathrm{MF}+\mathrm{Me}_{3} \mathrm{SiCF}_{3}+1 / 8 \mathrm{~S}_{8} \frac{\text { Diglyme or } \mathrm{THF}}{-60^{\circ} \mathrm{C} \text { to r.t., } 16 \mathrm{~h} \quad \mathrm{MSCF}_{3}+\mathrm{MeSiF}} \mathrm{M}=\mathrm{K}, \mathrm{Cs}, \mathrm{NMe}_{4} \text {, etc. }
$$

图式 4 一锅法制备 $\mathrm{MSCF}_{3}$

Scheme 4 One-pot synthesis of $\mathrm{MSCF}_{3}$

\subsection{2 早期直接亲核三氟甲硫基化反应}

早期的三氟甲硫基化法主要采用上述各种亲核性 的三氟甲硫基源，与烷基、芳基卤化物等亲电底物在高 温条件下实现三氟甲硫基的引入. 而为了解决亲核三氟 甲硫基源稳定性差的问题, 研究者们也发展了现场生成 亲核三氟甲硫基源的方法. 如 Remy 小组 ${ }^{[12]}$ 利用

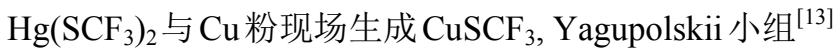
使用 $\mathrm{CF}_{3} \mathrm{SSCF}_{3}$ 与 $\mathrm{Cu}$ 粉现场产生 $\mathrm{CuSCF}_{3}$, 上海有机所 的陈庆云先生 ${ }^{[14]}$ 利用 $\mathrm{CuI}, \mathrm{Chen}$ 试剂与硫粉现场生成 $\mathrm{CuSCF}_{3}, \mathrm{Clark}$ 小组 ${ }^{[15]}$ 利用 $\mathrm{CSCl}_{2}$ 与 $\mathrm{KF} 、 \mathrm{NMe}_{4} \mathrm{~F}$ 现场生 成 $\mathrm{KSCF}_{3} 、 \mathrm{NMe}_{4} \mathrm{SCF}_{3}$, 来实现芳基碘化物和溴化物以 及活泼氯化物的三氟甲硫基化反应(Scheme 5).

$$
\begin{aligned}
& \mathrm{R}-\mathrm{I}(\mathrm{Br}, \mathrm{Cl})+\mathrm{MSCF}_{3} \stackrel{\text { Conditions }}{\longrightarrow} \mathrm{R}-\mathrm{SCF}_{3} \\
& \text { or in situ generated }
\end{aligned}
$$

图式 5 早期发展的亲核三氟甲硫基化反应

Scheme 5 Early development of nucleophilic trifluoromethylation

\subsection{3 过渡金属催化三氟甲硫基化反应}

从 20 世纪开始，三氟甲硫基化的研究走向了 “低 谷”, 在将近十年期间中, 关于三氟甲硫基化反应的报 道与突破相当之少. 而由于氟原子和三氟甲基在药物中 的应用, 在 2010 年前后, 三氟甲硫基化反应的研究又重 新受到了研究者们的热切关注, 过渡金属催化的三氟甲 硫基化反应得到了快速的发展.

2011 年, MIT 的 Buchwald 小组 ${ }^{[16]}$ 首次实现了过渡 金属 $\mathrm{Pd}$ 催化下芳基溴化物与 $\mathrm{AgSCF}_{3}$ 的交叉偶联反应, 重新将三氟甲硫基化反应带回了人们的视线. 他们小组 采用大位阻的 BrettPhos 作为配体, 成功实现了第一例 金属催化的交叉偶联反应(Scheme 6).

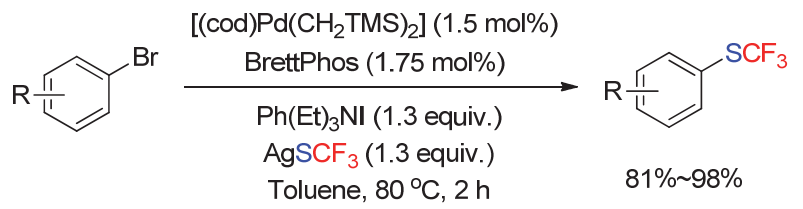

图式 $6 \mathrm{Pd}$ 催化芳基溴化物的三氟甲硫基化反应

Scheme 6 Palladium-Catalyzed coupling of aryl bromides with $\mathrm{AgSCF}_{3}$

2012 年 Lehigh University 的 Vicic 小组 ${ }^{[17]}$ 报道了镍 催化下芳基碘化物、溴化物与 $\mathrm{NMe}_{4} \mathrm{SCF}_{3}$ 的交叉偶联反 应. 该反应条件温和, 在室温下就可以以中等到优秀的
收率得到相应的三氟甲硫基化产物. 当底物中含有吸电 子取代基时，反应的产率会有所降低，对于活性更低的 芳基氯化物，反应则不能发生(Scheme 7).

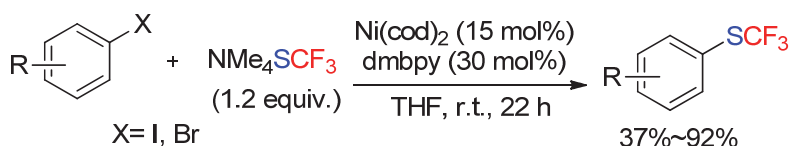

图式 $7 \mathrm{Ni}$ 催化芳基碘化物、溴化物三氟甲硫基化反应

Scheme 7 Nickel-Catalyzed coupling of ary iodides and bromides with $\mathrm{NMe}_{4} \mathrm{SCF}_{3}$

2015 年 RWTH Aachen University 的 Schoenebeck 小 组 ${ }^{[18]}$ 使用 $\mathrm{dppf}$ 作为配体, 实现了首例镍催化芳基氯化 物与 $\mathrm{NMe}_{4} \mathrm{SCF}_{3}$ 的交叉偶联反应 $($ Scheme 8).<smiles>CCOC(=O)Cc1c(C)n(C(=O)c2ccc(Cl)cc2)c2ccc(OC)cc12</smiles>
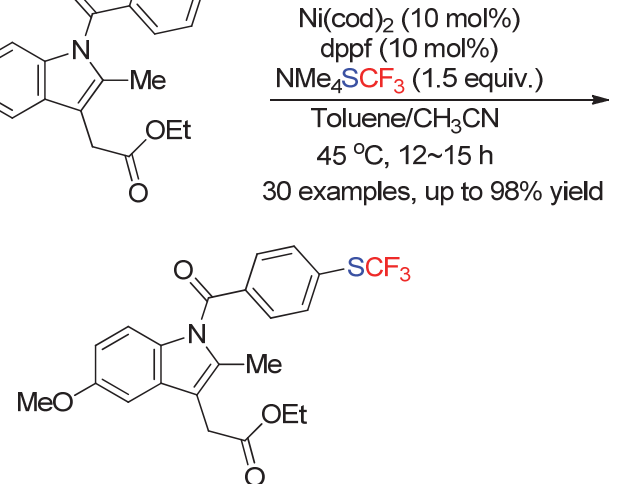

图式 $8 \mathrm{Ni}$ 催化芳基氯化物三氟甲硫基化反应

Scheme 8 Nickel-Catalyzed coupling of ary chlorides with $\mathrm{NMe}_{4} \mathrm{SCF}_{3}$

随后，该小组合成了双核的一价钯催化剂，并将其 应用到芳基碘化物、溴化物的三氟甲硫基化反应中，可 以获得优秀的反应收率 ${ }^{[19]}$ (Scheme 9).

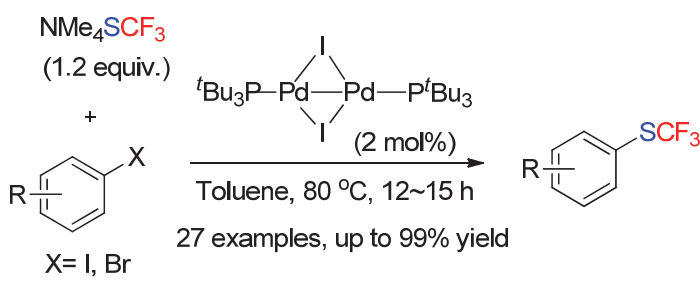

图式 9 双核一价 Pd 催化芳基碘化物、溴化物三氟甲硫基化反应 Scheme 9 Dinuclear palladium catalyzed coupling of ary iodides and bromides with $\mathrm{NMe}_{4} \mathrm{SCF}_{3}$

采用芳基氯化物相似的体系，她们小组使用芳基磺 酸酯、烯基磺酸酯等底物也成功实现了 $\mathrm{Ni}$ 催化下的交 叉偶联反应 ${ }^{[20]}$ (Scheme 10).

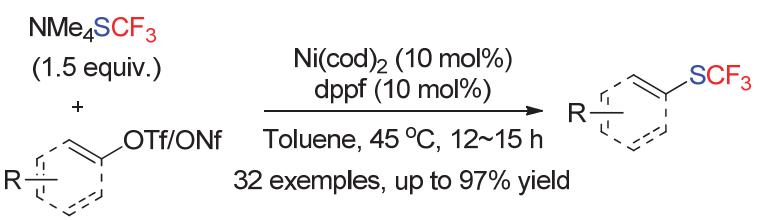

图式 $10 \mathrm{Ni}$ 催化芳基、烯基类卤化物的三氟甲硫基化反应 Scheme 10 Nickel-Catalyzed coupling of ary, vinyl triflates or nonaflates with $\mathrm{NMe}_{4} \mathrm{SCF}_{3}$ 
此外, 通过官能团化导向的策略, 可以更好地对底 物中的 $\mathrm{C}-\mathrm{Br}$ 键以及 $\mathrm{C}-\mathrm{Cl}$ 键进行活化, 从而可以更加 高效地实现相应的三氟甲硫基化转化. 不列颠哥伦比亚 大学的 Love 小组 ${ }^{[21]}$ 发现, 使用不同的含氮导向基, 来 使金属镍催化剂形成五元环金属中间体, 无需配体和添 加剂的加入, 便可以在温和的条件下实现 $\mathrm{C}-\mathrm{Br}$ 键、 $\mathrm{C}-\mathrm{Cl}$ 键到 $\mathrm{C}-\mathrm{SCF}_{3}$ 键的转化 (Scheme 11).

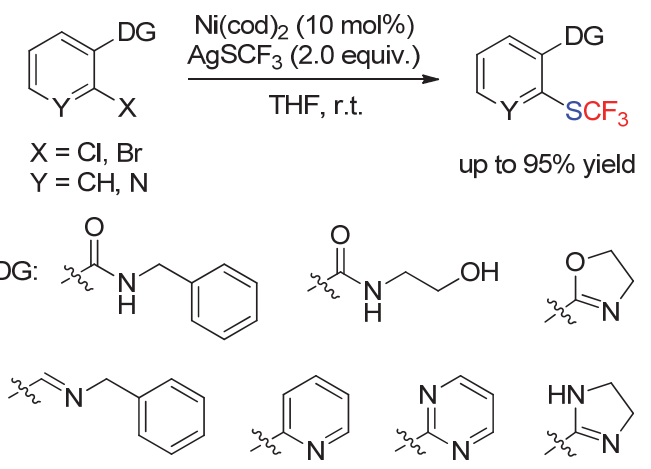

图式 11 官能团导向芳基 $\mathrm{C}-\mathrm{Br}$ 键、 $\mathrm{C}-\mathrm{Cl}$ 键的三氟甲硫基化反应 Scheme 11 Ligandless nickel-catalyzed ortho-selective directed trifluoromethylthiolation of aryl chlorides and bromides using $\mathrm{AgSCF}_{3}$

\subsection{4 亲核底物的氧化三氟甲硫基化反应}

亲核试剂与另一分子的亲核试剂在氧化性条件下 的偶联反应被称为氧化偶联反应. 而在三氟甲硫基领 域, 上海有机所的卿凤翎小组 ${ }^{[22]}$ 首先报道了该类转化. 他们使用芳基硼酸作为亲核试剂, 在硫粉和 Ruppert-Prakash 试剂为三氟甲硫基源, 碳酸银作为氧化 剂, 硫氰化亚铜作为催化剂的条件实现了该步转化 (Scheme 12).

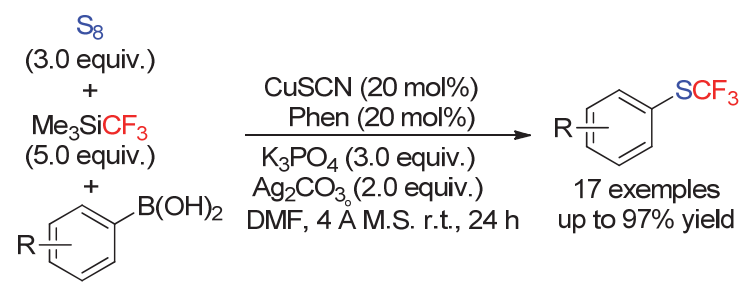

图式 12 铜催化嗍酸的氧化三氟甲硫基化反应

Scheme 12 Copper-Catalyzed oxidative coupling of aryl boronic acid with $\mathrm{TMSCF}_{3}$ and elemental sulfur

采用同样的策略, 该小组使用炔烃作为亲核试剂也 实现了无金属参与下的氧化偶联反应. 该反应无须惰性 气体保护，便可以在室温条件下顺利实现转化 ${ }^{[23]}$ (Scheme 13).

$$
\mathrm{R}=\underset{(6 \text { equiv.) }}{+\mathrm{S}_{8}+\mathrm{Me}_{3} \mathrm{SiCF}_{3} \text { equiv.) }} \frac{\mathrm{KF}(2.0 \text { equiv. })}{\mathrm{DMF} \text {, air, r.t., } 6 \mathrm{~h}} \mathrm{R}=\mathrm{SCF}_{3}
$$$$
22 \text { exemples, up to } 91 \% \text { yield }
$$

图式 13 炔烃的氧化三氟甲硫基化反应

Scheme 13 Metal-free oxidative coupling of terminal alkynes with $\mathrm{TMSCF}_{3}$ and elemental sulfur
2012 年, Vicic 小组 ${ }^{[24]}$ 使用 $\mathrm{NMe}_{4} \mathrm{SCF}_{3}$, 在 1 equiv. 的一价铜存在下，也实现了与芳基、烯基硼酸底物的氧 化三氟甲硫基化反应. 该反应无论对于吸电子或者给电 子取代基都可以取得良好到优秀的产率. 而对于反式的 烯基硼酸底物，可以得到构型保持的三氟甲硫基化产物 (Scheme 14).

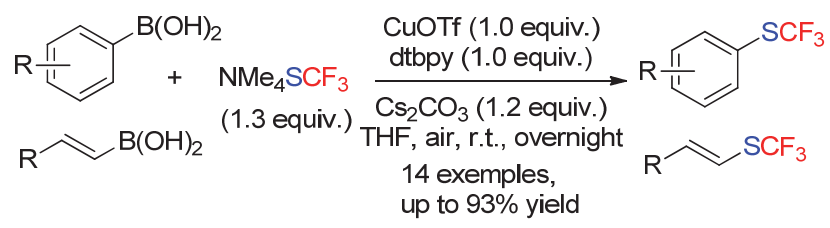

图式 14 铜介导的嗍酸氧化三氟甲硫基化反应

Scheme 14 Copper-mediated trifluoromethylthiolation of aryl boronic acids with $\mathrm{NMe}_{4} \mathrm{SCF}_{3}$

2013 年, 大连理工大学的段春迎小组 ${ }^{[25]}$ 改用硫粉 和 $\mathrm{CF}_{3} \mathrm{COONa}$ 的组合, 采用当量的铜盐和配体，也可以 实现嗍酸的氧化偶联反应，但由于 $\mathrm{CF}_{3} \mathrm{COONa}$ 脱羧需 要较高的温度, 因此该反应的温度较高. 2017 年，同济 大学赵晓明小组 ${ }^{[26]}$ 使用三氟甲硫基铜络合物, 在氧气 条件下实现了嗍酸底物的氧化偶联反应. 随后, 华东理 工大学的曹松小组 ${ }^{[27]}$ 使用 $\mathrm{AgSCF}_{3}$ 作为三氟甲硫基源并 且同时作为氧化剂, 实现铜催化下芳基硼酸的偶联反应 (Scheme 15).
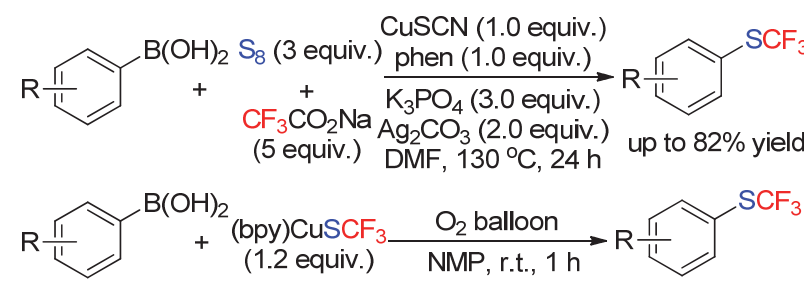
up to $92 \%$ yield

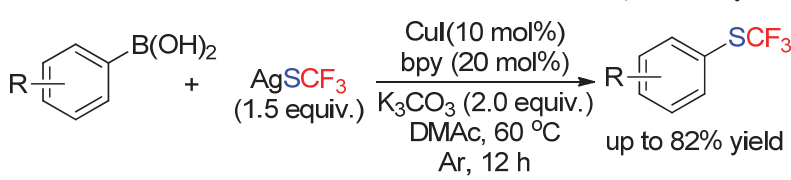

图式 15 嗍酸氧化三氟甲硫基化反应

Scheme 15 Oxidative trifluoromethylthiolation of boronic acids

使用亲核性的三氟甲硫基源，也可以在氧化条件下 实现 $\mathrm{C}-\mathrm{H}$ 键的三氟甲硫基反应(Scheme 16). 卿凤领小 组 ${ }^{[28]}$ 通过对氧化剂的考察, 发现在一价铜盐的催化下, 使用过氧化物作为氧化剂, 可以实现茮位 $\mathrm{C}-\mathrm{H}$ 键无官 能团导向的三氟甲硫基化反应. 北京大学的黄湧小 组 ${ }^{[29]}$ 报道了官能团导向芳基 $\mathrm{C}-\mathrm{H}$ 键的氧化三氟甲硫基 化反应. 该过程中, 氧化剂的作用可能是将转金属后的 $\mathrm{Pd}(\mathrm{II})-\mathrm{SCF}_{3}$ 中间体氧化成 $\mathrm{Pd}(\mathrm{IV})$ 中间体, 从而促进还原 消除过程. 中山大学的王洪根小组 ${ }^{[30]}$ 使用吡啶作为导 向基，在空气作为氧化剂的条件下实现了三价钴催化的 芳基 $\mathrm{C}-\mathrm{H}$ 键氧化三氟甲硫基化反应. 


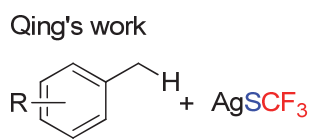

(6 equiv.)
CuTc (0.4 equiv.)

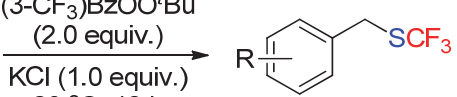

up to $92 \%$ yield
$\left(3-\mathrm{CF}_{3}\right) \mathrm{BzOO}^{t} \mathrm{Bu}$

Huang's work

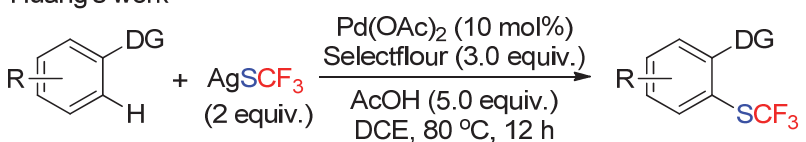
up to $91 \%$ yield

Wang's work

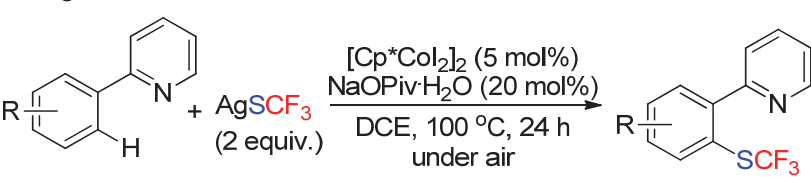

up to $65 \%$ yield

图式 16 氧化条件下 $\mathrm{C}-\mathrm{H}$ 三氟甲硫基化反应

Scheme 16 Oxidative trifluoromethylthiolation of $\mathrm{C}-\mathrm{H}$ bond

\subsection{5 稳定、易表征的三氟甲硫基金属络合物}

合成制备出稳定且容易表征的三氟甲硫基金属络 合物一方面可以解决该类三氟甲硫基源稳定性差且难 以表征的问题，另一方面，制备的络合物会更加有利于 研究该类反应的反应机理.

1992 年, Munavalli 小组 ${ }^{[31]}$ 使用 $\mathrm{CF}_{3} \mathrm{SSCF}_{3}$ 与铜粉在 乙腈中反应, 得到了一个晶体化合物, 当时他们认为该 结构是 $1: 1$ 的 $\mathrm{CuSCF}_{3}$ 和乙腈的配合物. 随后, 他们成 功对该络合物的单晶进行培养, 发现该单晶的结构是 $\left[\mathrm{CF}_{3} \mathrm{SCu}\right]_{10} \bullet 8 \mathrm{CH}_{3} \mathrm{CN}$, 这是首例可分离得到的三氟甲硫 基络合物 ${ }^{[32]}$.

2012 年, 福州大学的翁志强小组 ${ }^{[33]}$ 分离得到了双 齿氮配体络合的联吡啶单核铜络合物与菲罗琳双核铜 络合物. 该类络合物在空气和溶液中可以较为稳定的存 在. 随后他们也通过在体系中加入 $\mathrm{PPh}_{3}$, 合成了相应的 膦配体络合物 ${ }^{[34]}$. 2015 年, Vicic 小组 ${ }^{[35]}$ 使用 $\mathrm{PMe}_{3}$ 与 $\mathrm{CF}_{3} \mathrm{SO}_{2} \mathrm{Na}$ 反应, 首先观测到了 $\mathrm{CF}_{3} \mathrm{SSCF}_{3}$ 的生成. 他们 随后在反应体系中加入 $\mathrm{CuCl}$, 成功以 $80 \%$ 的氟谱收率 观测到了 $\mathrm{CuSCF}_{3}$ 的生成. 通过向反应体系中外加配体, 他们可以成功分离得到与翁志强小组相同的络合物. 同 样, 通过加入 $\mathrm{Ph}_{3} \mathrm{P}$ 和 $\mathrm{dppf}$, 他们也分离得到了单齿膦配 体和双齿膦配体络合的 $\mathrm{CuSCF}_{3}$ 的络合物 (Scheme 17).

采用该类可分离得到的络合物, 可以实现与各种芳

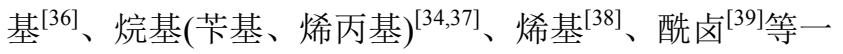
系列底物的亲核三氟甲硫基化反应.

\section{1 .6 其他一些类型的亲核三氟甲硫基化反应}

以上亲核反应中, 反应底物大多局限于卤化物. 而 在近年来, 其他亲电底物的三氟甲硫基化反应也得到了 发展.

芳基重氮盐 Sandmeyer 类型的三氟甲硫基化反应可 以直接通过 $\mathrm{AgSCF}_{3}$ 或 $\mathrm{CuSCF}_{3}$ 的反应来实现. 1975 年 Kondratenko 小组 ${ }^{[40]}$ 使用 $\mathrm{AgSCF}_{3}$ 与芳基重氮盐反应，可

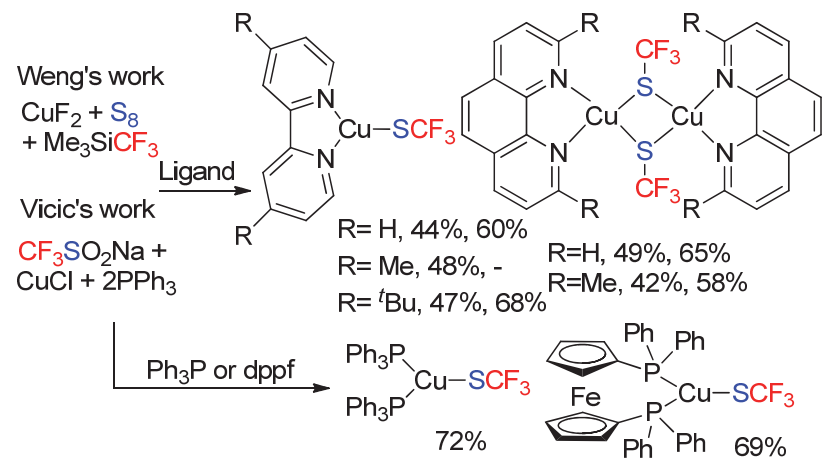

图式 $17 N, P$ 络合的 $\mathrm{CuSCF}_{3}$ 络合物

Scheme $17 N, P$-ligated $\mathrm{CuSCF}_{3}$ complex

以以中等的收率得到相应的三氟甲硫基化反应的产物, 这是 Sandmeyer 类型的三氟甲硫基化反应的首次报道. 在 2000 年, Clark 小组 ${ }^{[41]}$ 使用芳基重氮盐与 $\mathrm{CuSCF}_{3}$ 反 应, 实现了相应地转化, 且产率较 $\mathrm{AgSCF}_{3}$ 有所提高. 该 方法也可以从苯胺底物出发, 通过一锅法实现氨基到三 氟甲硫基的转化.

2014 年 Goossen 小组 ${ }^{[42]}$ 首先通过 Sandmeyer 类型的 硫氰化反应构建 $\mathrm{C}-\mathrm{S}$ 键，再通过 Ruppert 试剂形成的三 氟甲基负离子进攻氭基，实现了三氟甲硫基化的转化. 该方法避免了预先制备相对不稳定的 $\mathrm{CuSCF}_{3}$, 在产率 和底物兼容性上都有了很大的提升(Scheme 18).

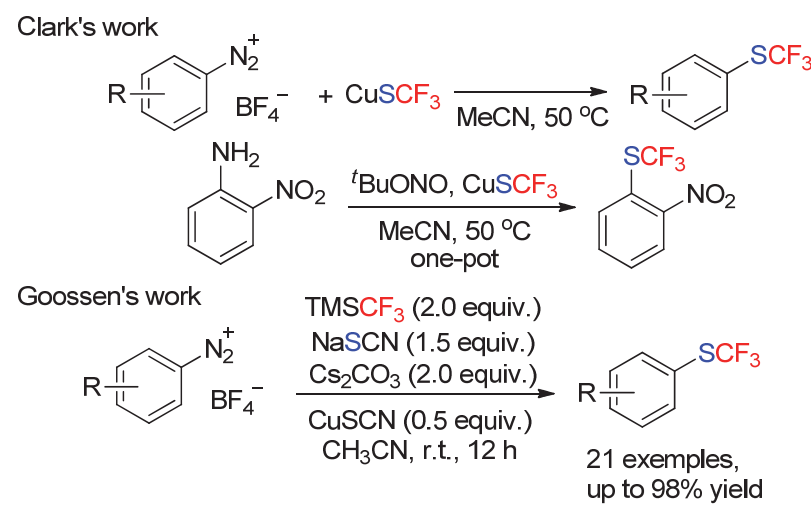

图式 18 Sandmeyer 类型的三氟甲硫基化反应 Scheme 18 Sandmeyer-type trifluoromethylthiolation

重氮甲酸酯可以在铜、钯等金属盐的存在下形成金 属卡宾, 该类卡宾有着很好的亲电性, 可以与各类亲核 试剂发生迁移插入反应.

在 2014 年，上海有机所的胡金波小组 ${ }^{[43 \mathrm{a}]}$ 和北京大 学的王剑波小组 ${ }^{[43 b]}$ 几乎同时报道了该类型的反应. 他 们采用了相同的策略, 首先以 $\mathrm{AgSCF}_{3}$ 和一价铜盐现场 生成 $\mathrm{CuSCF}_{3}$, 随后与 $\alpha$-重氮甲酸酯反应形成相应的铜 卡宾, 经迁移插入和质子淬灭后形成最终产物. 随后, Rueping 小组 ${ }^{[44]}$ 发现, 使用预先制备的 $\mathrm{CuSCF}_{3}$ 通过加 入水淬灭或者加入另一分子的亲电三氟甲硫基化试剂, 可以实现单/双三氟甲硫基化反应. Goossen 小组 ${ }^{[45]}$ 发现, 
无需预先制备 $\mathrm{CuSCF}_{3}$, 在 $\mathrm{CuSCN}$ 的催化下，使用 $\mathrm{Me}_{4} \mathrm{NSCF}_{3}$ 为三氟甲硫基源, 也可以实现相应的转化, 并且使用 $\mathrm{Me}_{4} \mathrm{NSeCF}_{3}$ 时，也可以实现三氟甲硒基化反 应(Scheme 19).

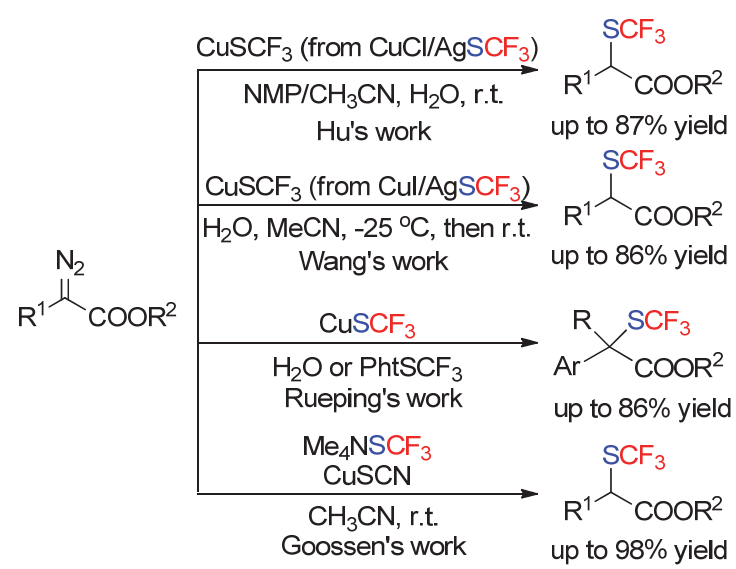

图式 19 重氮酸酯的三氟甲硫基化反应

Scheme 19 Trifluoromethylthiolation of diazo compounds through copper carbine migratory insertion

醇底物的亲核三氟甲硫基化反应由 Rueping 小组和 卿凤悀小组分别于 2014 年和 2015 年报道(Scheme 20). Rueping 小组 ${ }^{[46]}$ 使用 Lewis 活化着基的策略, 实现了活 化羟基底物如苠醇、烯丙醇的脱氧三氟甲硫基化反应. 卿风领小组 ${ }^{[47]}$ 发现, 醇羟基可以进攻硫代氟光气而转 变为更易离去的硫代氟甲酰氧基团, 使用过量的 $\mathrm{AgSCF}_{3}$ 可以进攻该基团, 从而实现未活化一级、二级醇 底物的三氟甲硫基化反应. 相比该两个小组的工作, 卿 凤翎小组的工作在底物范围上更广, 并且避免了 Lewis 酸的加入. 但是, 由于需要将羟基转变为离去基团, 首 先需要消耗等当量的硫代氟光气, 因而该反应一般需要 3 个当量以上的 $\mathrm{AgSCF}_{3}$ 以及大大过量的四丁基碘化铵.

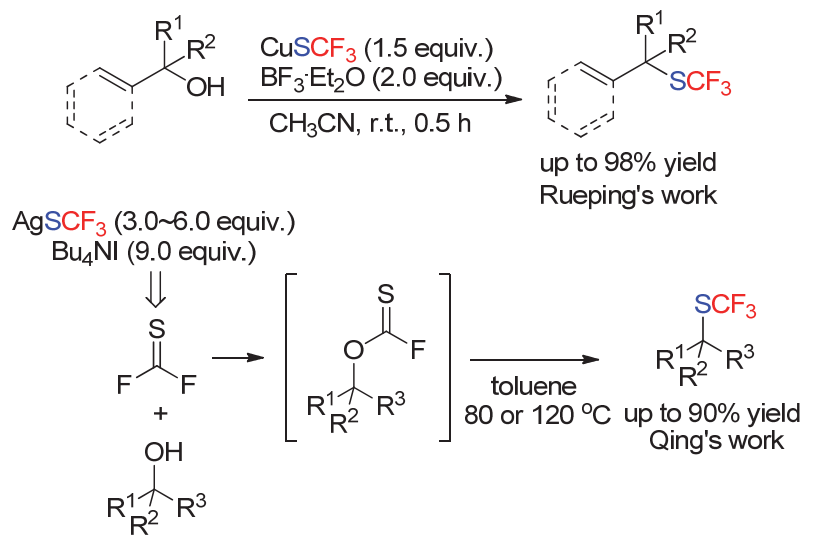

图式 20 醇底物的亲核三氟甲硫基化反应

Scheme 20 Dehydrotrifluoromethylthiolation of alcohols

兰州大学的梁永民小组 ${ }^{[8]}$ 采用炔丙醇底物, 使用 $\mathrm{BF}_{3} \cdot \mathrm{Et}_{2} \mathrm{O}$ 为 Lewis Acid, 实现了串联关环反应 (Scheme 21). 作者认为该反应的机理可能是底物在 Lewis Acid 的活化下首先离去羟基生成联烯三氟甲硫基结构. 随
后，在银盐的活化下，底物中的亲核试剂进攻双键，最 终水解生成最终产物.

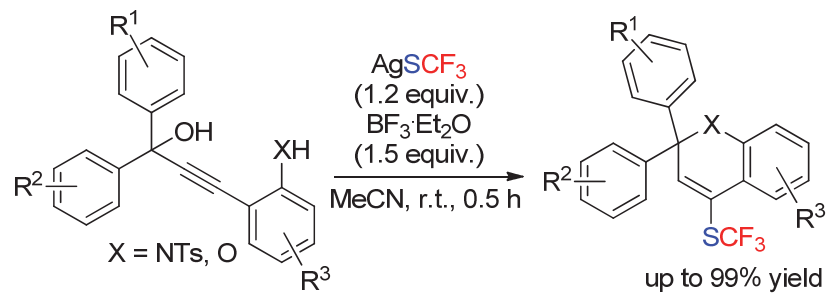

图式 21 炔丙醇底物的串联环化三氟甲硫基化反应

Scheme $21 \mathrm{AgSCF}_{3} / \mathrm{BF}_{3} \cdot \mathrm{Et}_{2} \mathrm{O}$ Mediated trifluoromethylthiolation cascade cyclization of propynols

将羟基转变为更容易离去的离去基团，也可以使用 亲核三氟甲硫基源来实现相应的三氟甲硫基转化. 如上 海有机所的游书力小组 ${ }^{[49]}$ 将羟基转变为碳酸酯, 可以 在钬催化下实现端位选择性的三氟甲硫基化反应.在该 反应中, 产生的支链产物会进一步转化为热力学稳定的 线性产物(Scheme 22).

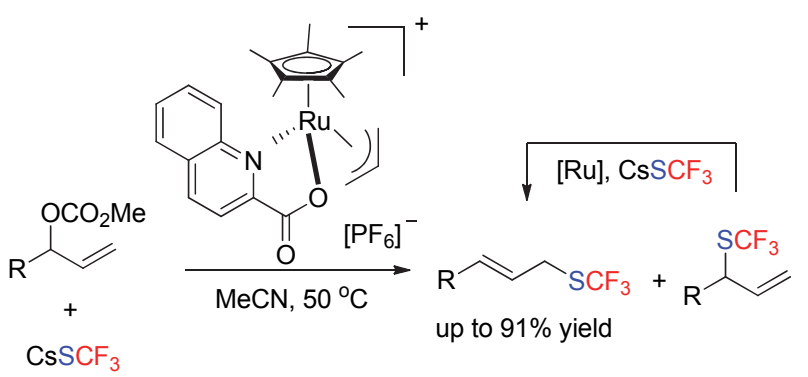

图式 22 钓催化区域端位选择性的烯丙基三氟甲硫基化反应

Scheme 22 Ruthenium-catalyzed regioselective allylic trifluoromethylthiolation reaction

马军安小组和 Cahard 小组 ${ }^{[00]}$ 合作, 实现了含三氟 甲硫基取代的手性胺以及手性酯类化合物的合成. 他们 将手性的氨基醇衍生为更容易离去的磺酸酯类化合物, 采用 $\mathrm{Me}_{4} \mathrm{NSCF}_{3}$ 为亲核三氟甲硫基源，通过一锅法的开 环、水解反应, 实现了该步转化, 合成了一类含三氟甲 硫基取代的结构新颖的化合物(Scheme 23).

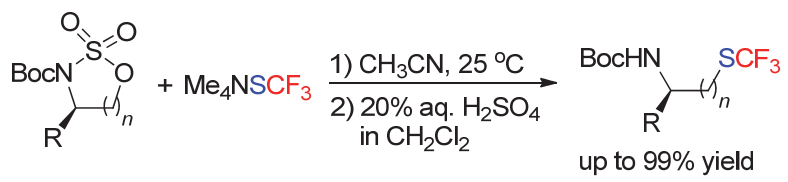

图式 23 环状磺酸酯类化合物的三氟甲硫基化反应

Scheme 23 Trifluoromethylthiolation of cyclic sulfamidates

二芳基高价碘盐也是一类高活性的亲电试剂，其相 关的亲核三氟甲硫基化反应也有相关报道(Scheme 24). 2016 年, Rueping 小组 ${ }^{[51]}$ 使用 $\mathrm{CuSCF}_{3}$, 通过与芳基均三 甲基苯基高价碘盐实现了区域选择性的三氟甲硫基化 反应. 张成潘小组 ${ }^{[52]}$ 使用炔基苯基高价碘盐为亲电试 剂, $\mathrm{Me}_{4} \mathrm{NSCF}_{3}$ 为亲核试剂, 实现了炔烃端选择性的三 氟甲硫基化反应。此外，作者采用相应的 $\mathrm{Me}_{4} \mathrm{NSeCF}_{3}$, 
也可以实现三氟甲硒基化反应.

$$
\begin{gathered}
\mathrm{CuSCF}_{3} \\
\begin{array}{c}
(1.5 \text { equiv. }) \\
70^{\circ} \mathrm{C}, 1.5 \mathrm{~h}
\end{array}
\end{gathered}
$$

图式 24 高价碘盐的三氟甲硫基化反应

Scheme 24 Trifluoromethylthiolation of Unsymmetrical $\lambda^{3}$-iodane derivatives

除去 $\mathrm{AgSCF}_{3}$ 中三氟甲硫基的亲核性外，银也具有 一定的 Lewis 酸性, 而利用该两点, 研究者们也使用 $\mathrm{AgSCF}_{3}$ 来捕捉反应体系中生成的活性中间体. 2013 年 Lee 小组 ${ }^{[53]}$ 从 $\mathrm{AgSCF}_{3}$ 出发, 一方面利用 $\mathrm{Ag}$ 离子的 Lewis 酸性来活化三炔底物来形成苯炔中间体, 另一方 面利用 $\mathrm{SCF}_{3}$ 阴离子的亲核性来进攻苯炔中间体, 从而 实现串联成环反应. 最近作者发现, 当炔丙位有氢原子 存在时，该反应会优先发生 Alder-ene 反应从而形成烯联烯中间体，随后受 $\mathrm{AgSCF}_{3}$ 进攻，并在三氟乙醇存在 下通过后续的迁移重排反应来实现串联环化反应 ${ }^{[54]}$ (Scheme 25).

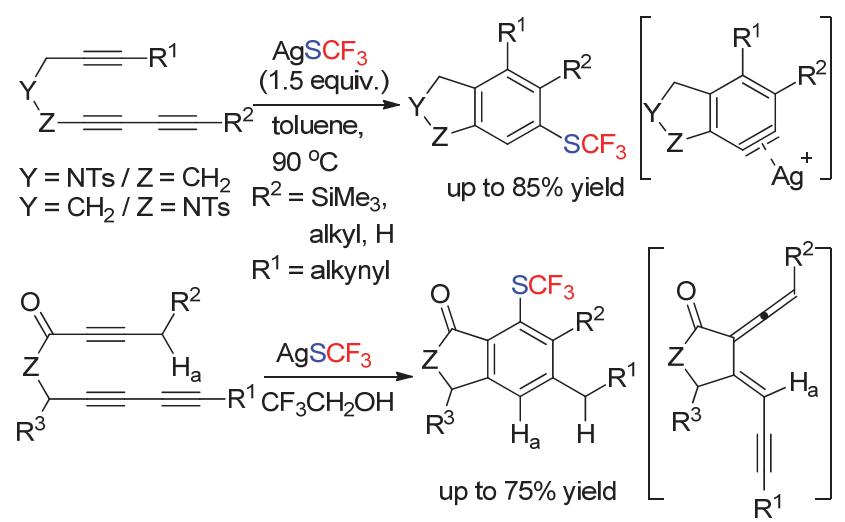

图式 $25 \mathrm{AgSCF}_{3}$ 与苯炔、烯炔中间体的反应

Scheme 25 Trifluoromethylthiolation of aryne and allene-enyne intermediate

2014 年, 复旦大学的吴劼小组 ${ }^{[5]}$ 从肜底物出发, 使 用 $\mathrm{AgOTf}$ 为催化剂, 对甲氧基磺酰氯作为活化剂, 首先 形成氧化异喹啉中间体, 随后被 $\mathrm{AgSCF}_{3}$ 捕捉, 在碱促 进下形成三氟甲硫基化异喹啉产物. 2016 年, 上海有机 所的胡金波小组 ${ }^{[56]}$ 使用 $\mathrm{AgSCF}_{3}$ 为亲核试剂, 炔基碘作 为亲电试剂, 实现了苯炔前体的碘化-三氟甲硫基化反 应(Scheme 26).

另外，在 2013 年 Zard 小组 ${ }^{[57]}$ 设计合成了一类可以 现场生成三氟甲硫基阴离子的试剂(Scheme 27). 该试 剂可以用于高效地制备三氟甲硫基取代的芦竹碱和 $\alpha$ 三氟甲硫基取代的酮类化合物.

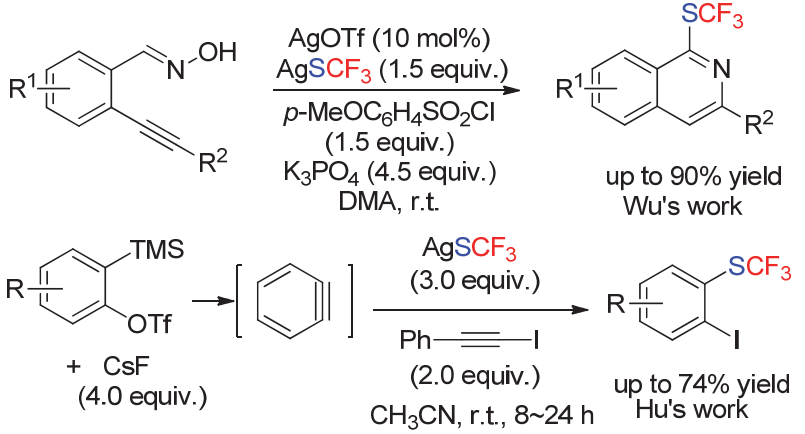

图式 $26 \mathrm{AgSCF}_{3}$ 与氧化异喹啉、苯炔中间体的反应

Scheme 26 Trifluoromethylthiolation of $\mathrm{N}$-oxide and aryne intermediate

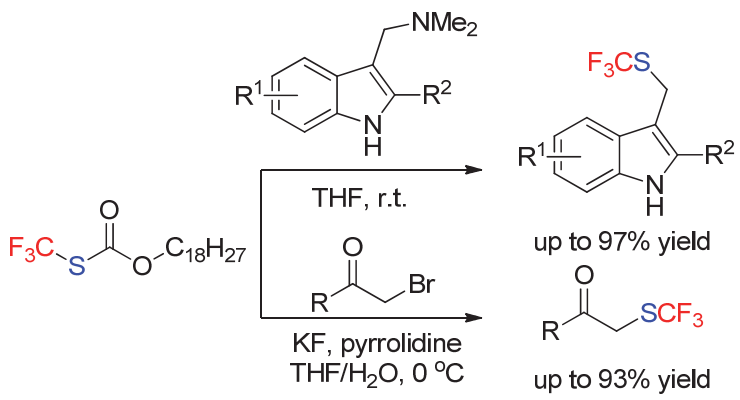

图式 27 现场生成三氟甲硫基阴离子的反应

Scheme $27 \quad$ In-situ generation and capture of trifluoromethanethiol

随后，上海有机所施敏课题组 ${ }^{[58]}$ 采用该试剂，在不 同溶剂的条件下实现了 MBH 酯底物区域选择性的三氟 甲硫基化反应. 随后，作者同样采用 $\mathrm{MBH}$ 酯底物，实现 了分子内的成环反应, 并且通过手性三级胺的催化, 实 现了不对称二氟甲硫基片段的引入 ${ }^{[59]}$ (Scheme 28). 该

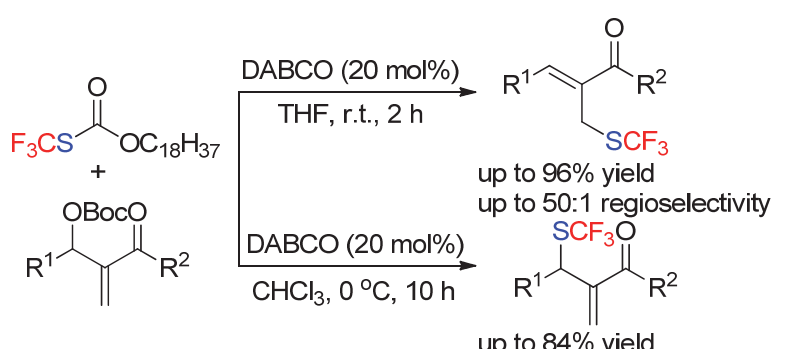

up to $>99: 1$ regioselectivity

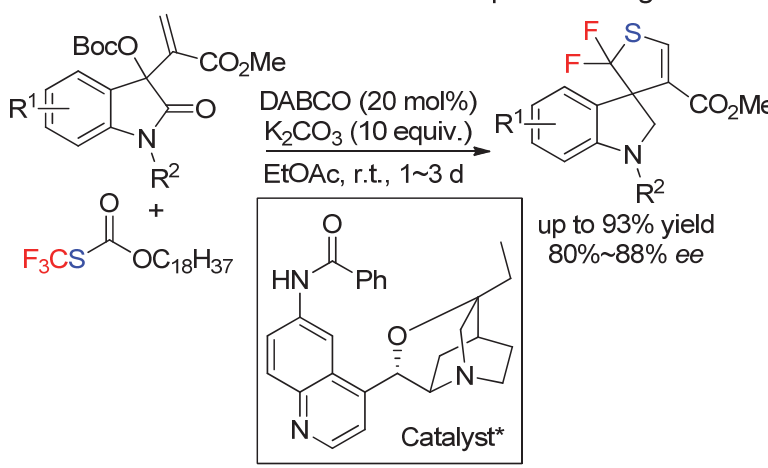

图式 $28 \mathrm{MBH}$ 酯的亲核三氟甲硫基化反应

Scheme 28 Nucleophilic trifluoromethylthiolation of Morita-BaylisHillman carbonates 
产物的机理可能是由 $\mathrm{MBH}$ 反应的中间体在碱性条件下 拔氢，与三氟甲硫基阴离子现场产生的氟光气反应生成 的中间体通过后续的反应形成的.

\section{2 亲电三氟甲硫基化反应}

相比较于亲核三氟甲硫基化反应，亲电三氟甲硫基 化反应由于早期试剂的贵乏, 其相应的研究报道较少. 在 20 世纪 60 年代开始, 关于 $\mathrm{CF}_{3} \mathrm{SCl}$ 和 $\mathrm{CF}_{3} \mathrm{SSCF}_{3}$ 的亲 电三氟甲硫基化反应开始被报道.

$\mathrm{CF}_{3} \mathrm{SCl}$ 有着很高的亲电三氟甲硫基化活性, 其反 应报道较多, 可以实现一系列富电子底物如富电子芳 烃、杂芳烃、活泼亚甲基底物、有机金属试剂、烯醇硅 醚、烯胺等亲电三氟甲硫基化反应 ${ }^{[60]}$ (Scheme 29). 但在 上述反应中, 对于底物类型的研究只有有限的个别例 子, 并没有进行系统的研究. 其次, 由于 $\mathrm{CF}_{3} \mathrm{SCl}$ 高的反 应活性, 反应的选择性通常较差, 且需要在低温下进行 反应操作. 更重要的是, $\mathrm{CF}_{3} \mathrm{SCl}$ 有着很高的毒性, 因而 限制了其广泛使用.

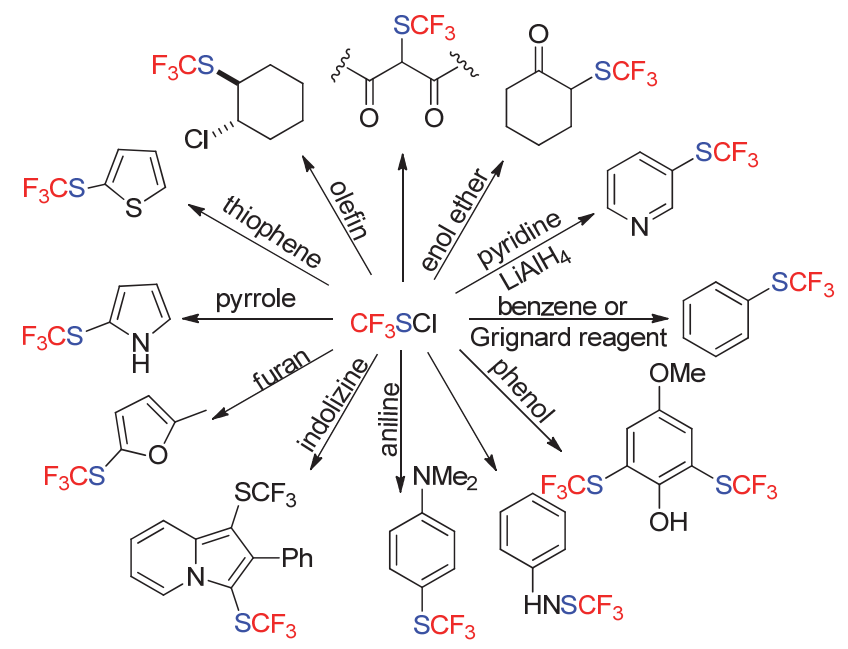

图式 $29 \mathrm{CF}_{3} \mathrm{SCl}$ 的亲电三氟甲硫基化反应

Scheme 29 Electrophilic trifluoromethylthiolation using $\mathrm{CF}_{3} \mathrm{SCl}$

与 $\mathrm{CF}_{3} \mathrm{SCl}$ 相比, $\mathrm{CF}_{3} \mathrm{SSCF}_{3}$ 的活性较低, 其相关反 应报道较少，它能与活泼亚甲基类底物、芳基钠等实现 相应的三氟甲硫基化反应 ${ }^{[61]}$. 在 2014 年, Daugulis 小 组 ${ }^{[62]}$ 利用 8-氨基喹啉作为导向基, 在醋酸铜的催化下实 现了首例 $\mathrm{C}-\mathrm{H}$ 活化三氟甲硫基化反应(Scheme 30).

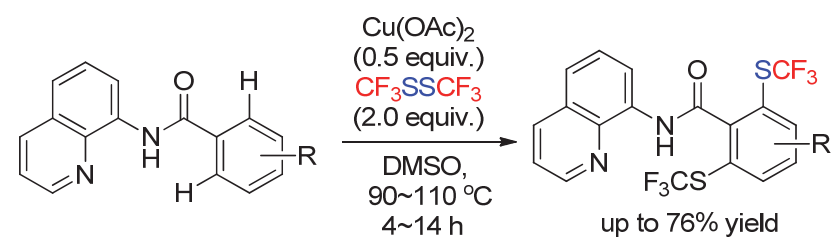

图式 30 8-氨基喹啉导向 $\mathrm{C}-\mathrm{H}$ 三氟甲硫基化反应

Scheme 30 Auxiliary-assisted trifluoromethylthiolation using $\mathrm{CF}_{3} \mathrm{SSCF}_{3}$

虽然该两种亲电三氟甲硫基化试剂都有着出色的 反应性能, 但是由于其都是高毒性气体 ${ }^{[63]}$, 给操作带来
了诸多不便，也因而限制了其应用与发展. 因此，发展 新型、高活性且便于操作的亲电三氟甲硫基化试剂成为 了该领域迫切需要解决的问题.

从 2008 年开始, 一系列便于操作、稳定的亲电三氟 甲硫基化试剂及方法逐渐被开发并且得到了应用，并对 于各类底物的三氟甲硫基化反应都进行了较为系统的 研究. 此外, 也有着基于手性骨架的亲电三氟甲硫基试 剂的相关报道，为不对称引入三氟甲硫基团提供了新的 策略. Scheme 31 列举了文献中已有的亲电三氟甲硫基 化试剂及方法，下面将按照不同的试剂逐一进行介绍.
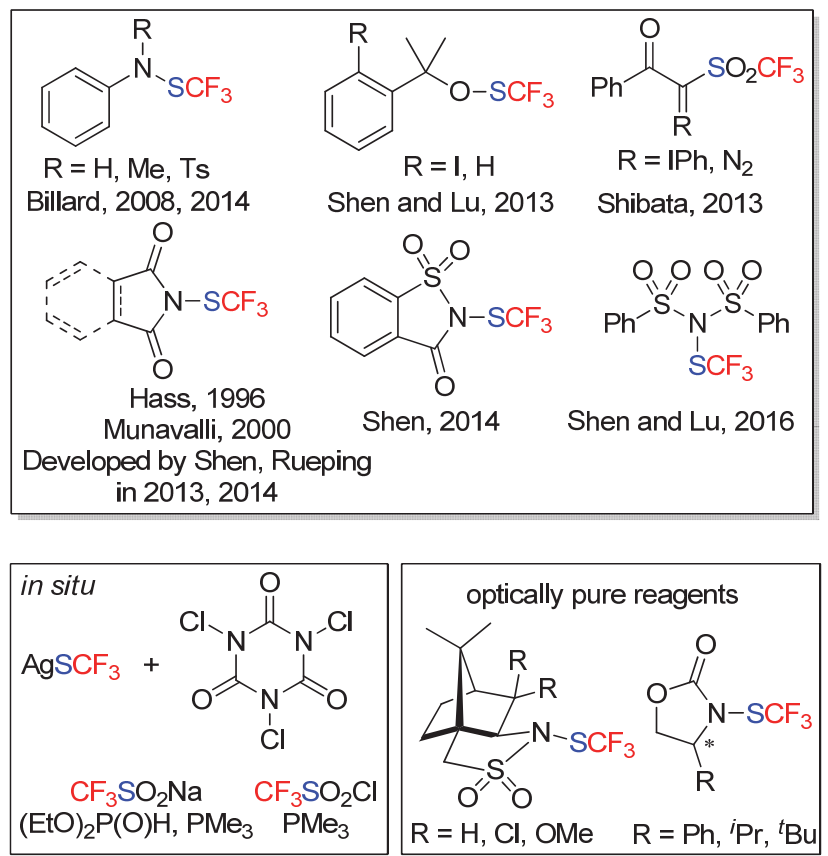

图式 31 亲电三氟甲硫基试剂种类

Scheme 31 Different types of electrophilic trifluoromethylthiolation reagents

\subsubsection{Billard 试剂}

在 2008 年, 法国 Billard 和 Langlois 等 $^{[64]}$ 利用二乙 胺基三氟化硫(DAST)、Ruppert 试剂( $\left.\mathrm{TMSCF}_{3}\right)$ 制备了含 三氟甲硫基的苯胺类衍生物. 该衍生物进一步与碘甲烷 发生 $\mathrm{S}_{\mathrm{N}} 2$ 反应，可进一步制备含有甲基取代的 Billard 试 剂。该小组通过后续的研究发现, 该类试剂可以在 Lewis Acid 或者 Brønsted Acid 的活化下实现烯烃 ${ }^{[65]}$ 、炔

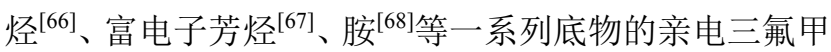
硫基化反应(Scheme 32).

同时，他们也发现，在合成该苯胺骨架的试剂过程 中, 当底物的胺含有吸电子取代基时会优先生成一个亚 胺中间体，该中间体可以在三氟乙酸的水解下，进一步 生成活性更高的第二代 Billard 试剂 ${ }^{[64]}$. 在 2014 年, 他 们对该类试剂的反应活性进行了报道, 发现该类试剂相 比第一代苯胺结构的试剂有着更为出色的反应活性, 除 去上述报道的反应外还可以实现活泼 $\mathrm{C}-\mathrm{H}$ 键底物 ${ }^{[69]}$ 、 杂环底物 $\mathrm{C}-\mathrm{H}$ 键底物 ${ }^{[70]}$ 、硼酸底物 ${ }^{[71]}$ 、杂原子亲核试 


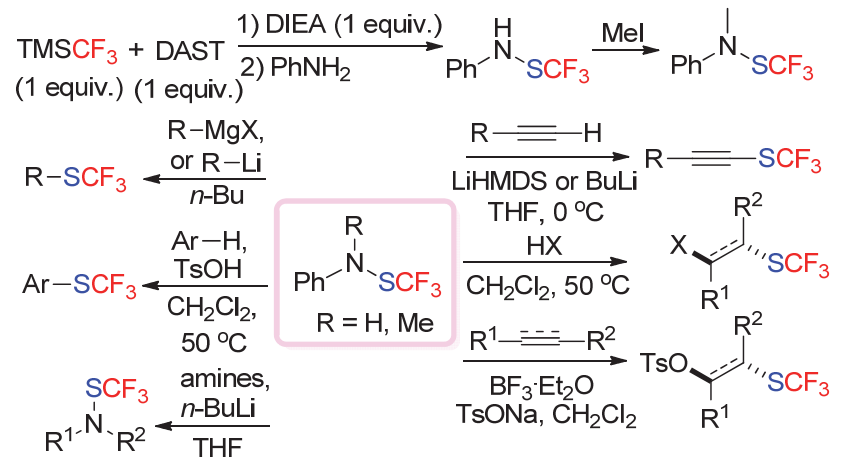

图式 32 第一代 Billard 试剂的制备及反应

Scheme 32 Preparation and reactions of first generation of Billard reagent

剂 $^{[72]}(\mathrm{S}, \mathrm{O})$ 、活泼卤化物(类卤化物)极性反转的三氟甲硫 基化反应 ${ }^{[73]}$ 、炔烃的单、双三氟甲硫基化反应 ${ }^{[74]}$ 以及富 电子芳烃、杂芳烃底物的 $\mathrm{C}-\mathrm{H}$ 键三氟甲硫基化反应 ${ }^{[75]}$ (Scheme 33).

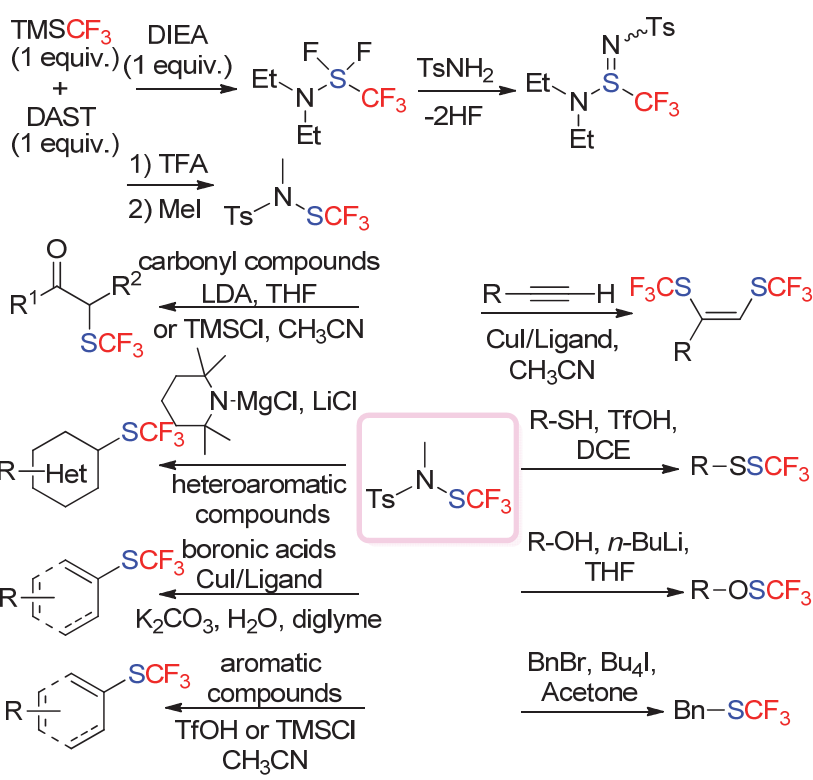

图式 33 第二代 Billard 试剂的制备及反应

Scheme 33 Preparation and reactions of Second generation of Billard reagent

最近 Billard 小组 ${ }^{[76]}$ 也发现, 采用第二代活性更高 的试剂, 可以在超强酸的作用下对该试剂进行进一步的 活化, 从而可以在低温下对一系列芳胺底物实现芳环上 的三氟甲硫基化反应(Scheme 34). 作者也通过核磁手 段, 观察到了活性中间体的产生. 作者认为, 采用该方 法, 可以在合成设计的后期方便地在芳环上引入三氟甲 硫基官能团.

在 Billard 小组工作的基础上，其他课题组采用不同 的酸活化剂，实现了不同类型的亲电三氟甲硫基化反 应. 2013 年上海有机所的卿凤翎小组 ${ }^{[77]}$ 使用色胺底物, 分别在 Lewis Acid 和 Brønsted Acid 活化的条件下实现

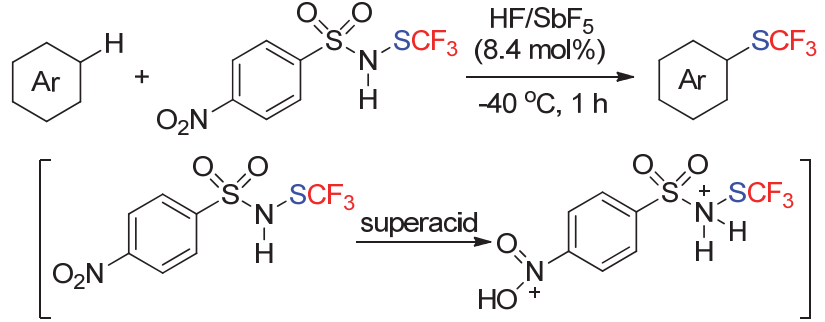

图式 34 超强酸催化下芳胺底物的三氟甲硫基化反应

Scheme 34 Superacid-catalyzed trifluoromethylthiolation of aromatic amines

了吲哚 2 位三氟甲硫基化以及串联关环三氟甲硫基化反 应. 随后，他们采用乙酰氯作为 Lewis Acid, 实现了烯 丙基硅试剂的三氟甲硫基化反应 ${ }^{[78]}$. 而同样采用酰氯 活化的策略，上海有机所的刘国生小组 ${ }^{[78 b]}$ 实现了钯催 化条件下吡啶导向的 $\mathrm{C}-\mathrm{H}$ 键三氟甲硫基化反应. 华东 理工大学曹松小组 ${ }^{[78 c]}$ 采用乙酰氯活化的策略, 发现对 于酮类化合物，可以很好地实现羰基 $\alpha$ 位的三氟甲硫基 化反应(Scheme 35).

Qing's work<smiles>[R]OC(=O)NCCc1c[nH]c2ccccc12</smiles>
Liu's work

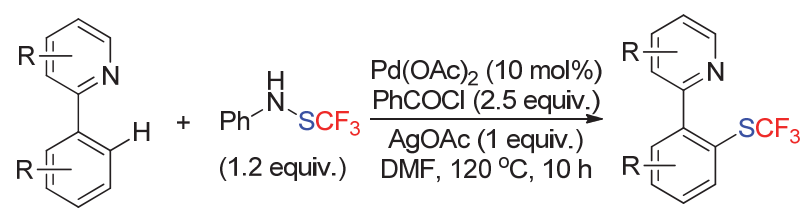

Cao's work up to $91 \%$ yield

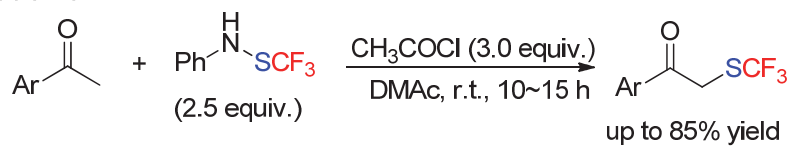

图式 35 酸和酰氯活化下的三氟甲硫基化反应

Scheme 35 Trifluoromethylthiolation under the activation of acid or acyl chloride using the first generation of Billard reagent

复旦大学的吴劼小组发现 $\mathrm{BiCl}_{3}$ 可以作为 Lewis Acid 来高效地对该试剂进行活化, 从而实现一系列串 联关环反应，成功构建了苯并 $[1,2]$ 噻嗪 1,1-二氧化

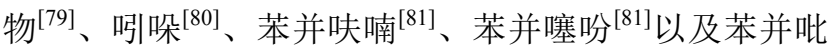
喃类衍生物 ${ }^{[82]}$. 此外, 炔基硅底物 ${ }^{[83]}$ 也可以在该条件 下，实现相应的三氟甲硫基化转化(Scheme 36). 


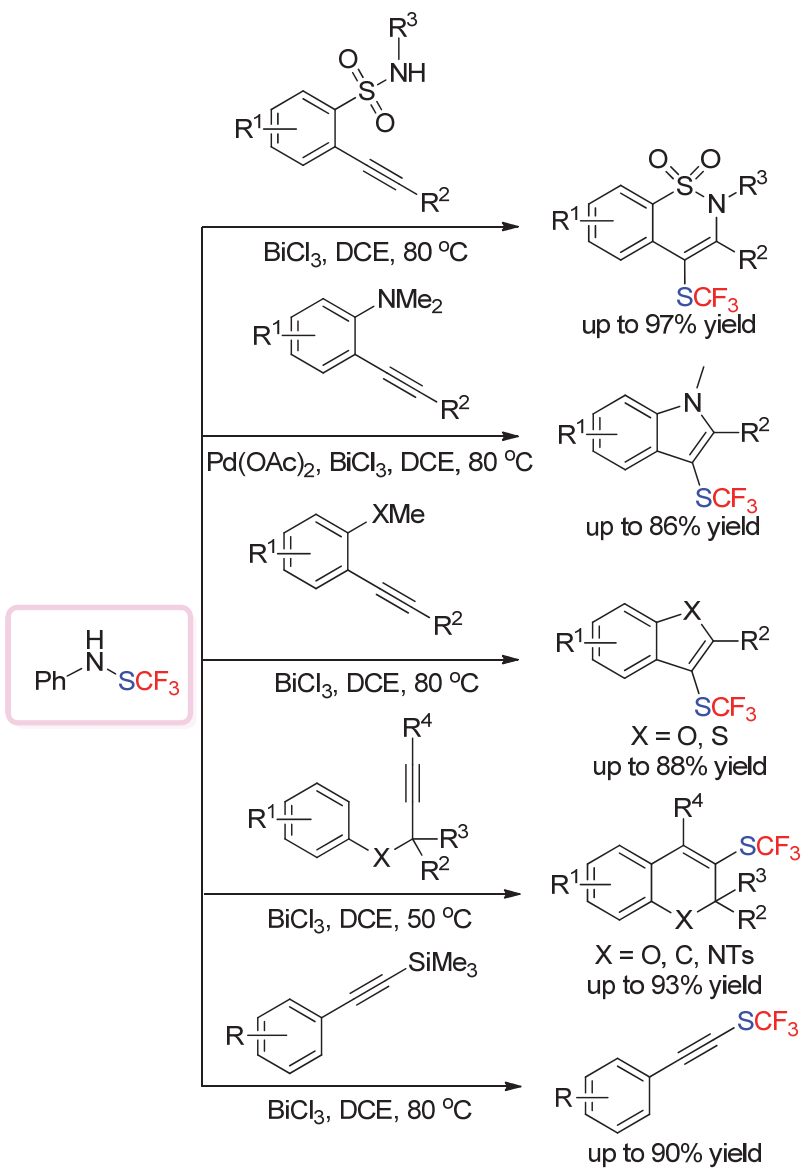

图式 36 吴款小组的工作

Scheme 36 Wu's Work using the first generation of Billard reagent

嘉兴学院的邱观音生小组与上海海洋大学的盛洁 小组 ${ }^{\left[{ }^{[4]}\right.}$ 合作, 从磺酰氯出发, 现场生成亚磺酸钠盐后, 使用对甲苯磺酸为酸添加剂对试剂进行活化, 可以实现 砜基三氟甲硫基产物的合成(Scheme 37).

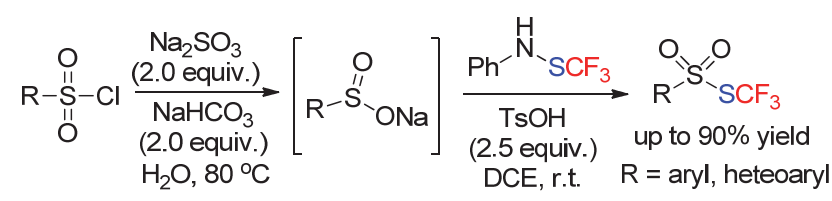

图式 37 磺酰氯的一锅法三氟甲硫基化转化

Scheme 37 One-pot reaction of sulfonyl chloride and Billard reagent

\subsubsection{Lu and Shen 试剂}

在 2013 年, 上海有机所的吕龙和沈其龙小组 ${ }^{\left[{ }^{[5]}\right.}$ 在 试图发展基于 Togni 试剂类型的亲电三氟甲硫基试剂过 程中, 合成了一类新颖结构的亲电三氟甲硫基化试剂. 2014 年, MIT 的 Buchwald 小组 ${ }^{[86]}$ 通过对该类底物的衍 生化, 合成了固体状态的衍生物, 发现在该衍生物的结 构中, 三氟甲硫基是与氧原子相连. 随后他们通过 MOF 技术, 将该试剂吸附并通过 $\mathrm{X}$ 单晶射线衍射, 确认该结 构并不是类似于 Togni 试剂而是三氟甲基次磺酸酯的结 构(Scheme 38).

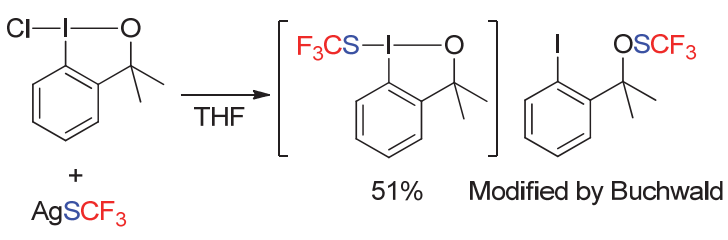

图式 $38 \mathrm{Lu}$ and Shen 试剂的合成及结构修正

Scheme 38 Preparation and modify of Lu and Shen reagent

采用该试剂, 吕龙和沈其龙小组 ${ }^{[85]}$ 首先实现了 $\beta$-酮 酸酯、芳基硼酸、炔烃以及羰基 $\alpha$ 位 $\mathrm{C}-\mathrm{H}$ 键的三氟甲 硫基化反应(Scheme 39). 在芳基硼酸的反应基础上, 通 过提高反应温度，他们可以实现烷基硼酸的三氟甲硫基 化反应 ${ }^{[87]}$. 随后, 他们也发现, 该试剂可以在 Brønsted Acid 的活化下实现吲哚底物的三氟甲硫基化反应 ${ }^{[88]}$. 此外, 他们小组对该类试剂的结构活性相关做了比较, 发现该试剂中的碘原子在大多数反应情况下对反应并 没有产生明显的差别. 采用不带碘的试剂, 他们在醋酸 作为反应溶剂的条件下, 实现苯基亚磺酸钠底物的三氟 甲硫基化反应 ${ }^{[89]}$. 此外, 在 $\beta$-酮酸酯的反应基础上, 作 者在奎宁作为碱或奎宁定作为相转移催化剂的条件下 实现了 $\beta$-酮酸酯、吲哚酮底物的不对称三氟甲硫基化反 应 $^{[90]}$ (Scheme 40).

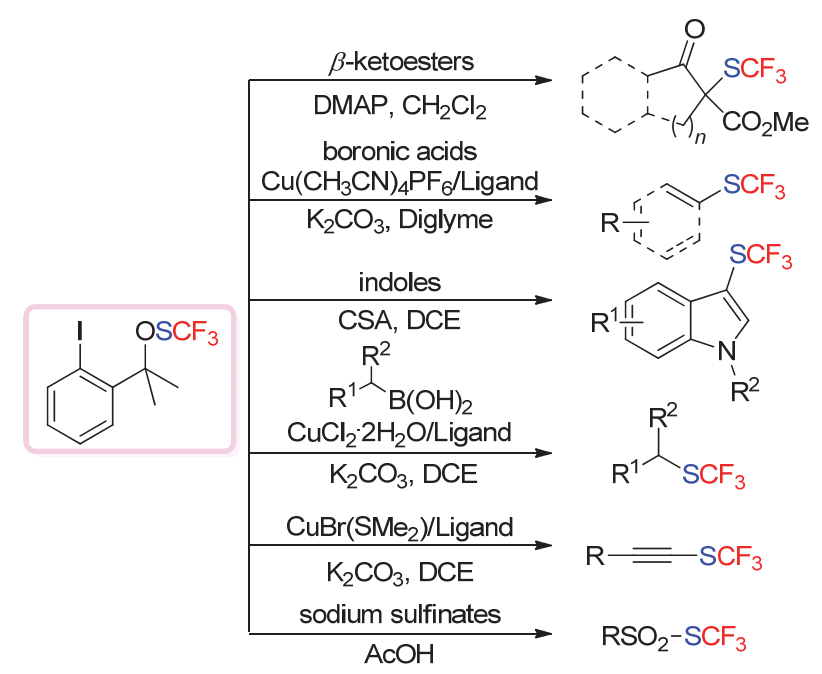

图式 $39 \mathrm{Lu}$ and Shen 试剂的反应

Scheme 39 Reactions of $\mathrm{Lu}$ and Shen reagent

2014 年, Gade 小组 ${ }^{[1]}$ 采用该试剂使用 Boxmi 作为 配体, 在铜催化下实现了 $\beta$-酩酸酯的反应(Scheme 41). 该反应体系无论对于 5 元环还是 6 元环都可以适用, 且 不需要大位阻的金刚烷基取代基.

浙江师范大学朱向明小组 ${ }^{[92]}$ 在硫糖苷的活化中, 使用该亲电三氟甲硫基化试剂活化糖苷键，实现了醇的 糖苷化反应(Scheme 42). 在该反应中, 作者认为, 该试 剂在三氟磺酸三甲基硅酯作为 Lewis Acid 的活化下, 接 受硫原子进攻，可以形成更易离去的硫鎓盐中间体，最 后实现糖苷化反应. 


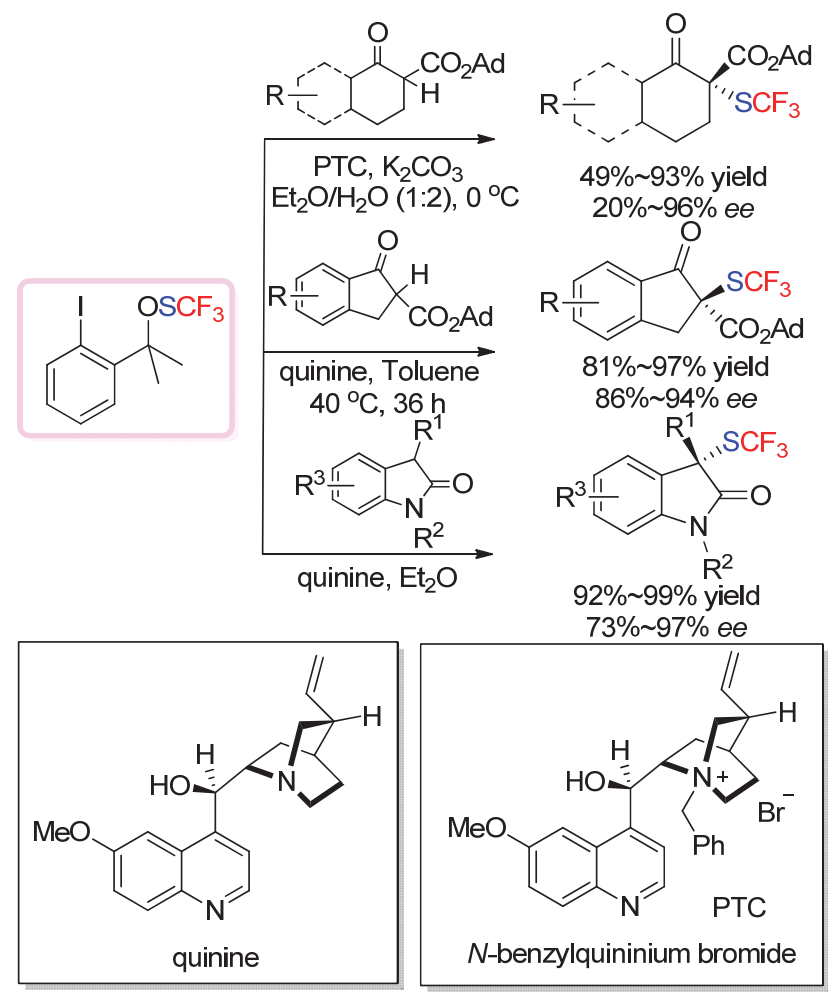

图式 40 不对称三氟甲硫基化反应

Scheme 40 Enantioselective electrophilic trifluoromethylthiolation using Lu and Shen reagent

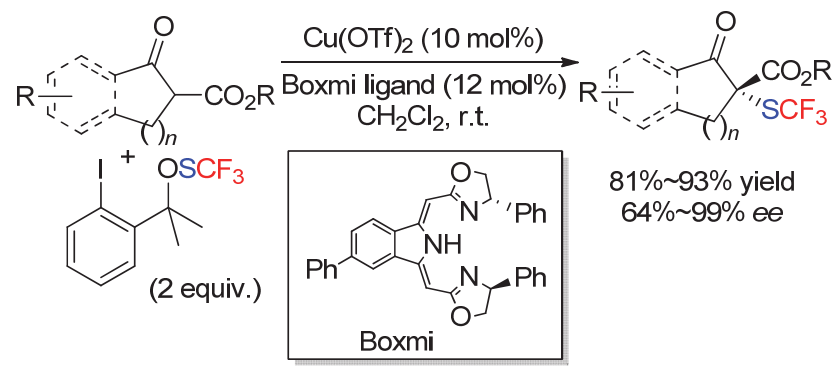

图式 $41 \mathrm{Cu}(\mathrm{OTf})_{2} /$ boxmi 体系下不对称三氟甲硫基化反应

Scheme 41 Enantioselective electrophilic trifluoromethylthiolation using $\mathrm{Lu}$ and Shen reagent and copper-boxmi complexes

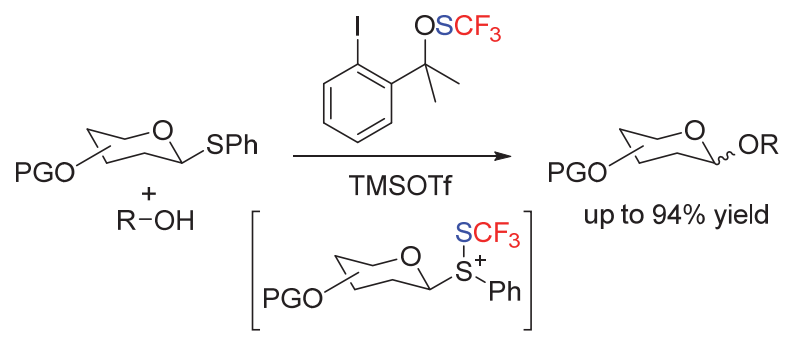

图式 42 糖苗键的活化

Scheme 42 Thioperoxide-mediated activation of thioglycoside donors

普渡大学的戴明骥小组 ${ }^{\left[{ }^{[3]}\right.}$ 采用环丙醇底物, 在铜 催化条件下通过开环三氟甲硫基化反应，构建了 $\beta$ 位三
氟甲硫基取代的羰基化合物(Scheme 43). 作者认为该 反应的机理可能是生成的重排后的烷基铜物种与亲电 试剂发生偶联进而生成最终产物.

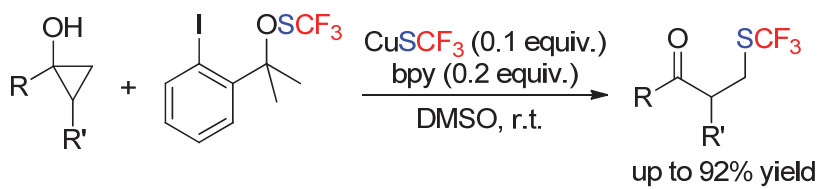

图式 43 铜催化环丙醇亲电开环三氟甲硫基化反应

Scheme 43 Copper-catalyzed electrophilic ring-opening cross-coupling of cyclopropanols

通过吕龙和沈其龙小组的研究还发现，该试剂除去 实现亲电三氟甲硫基化反应以外，还可以作为体系中自 由基的淬灭剂，实现自由基类型的三氟甲硫基化反应， 我们将在自由基反应中进行介绍.

\subsubsection{Shibata 试剂}

2013 年, 日本的 Shibata 小组 ${ }^{[94]}$ 发展了一种基于高 价碘叶立德类型的亲电三氟甲硫基化试剂. 该试剂的合 成较为廉价, 从三氟亚磺酸钠出发与溴代苯乙酮反应, 随后在等当量的醋酸碘苯的存在下，可以以当量的收率 获得该试剂(Scheme 44).

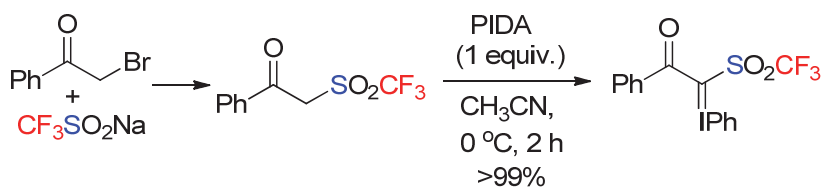

图式 44 Shibata 试剂的制备

Scheme 44 Preparation of Shibata reagent

采用该试剂, 他们小组首先实现了 $\beta$-酮酸酯、吲哚 衍生物、烯胺类底物在铜催化下的反应 ${ }^{[94]}$. 随后, 他们 利用该试剂，也实现了铜催化下硼酸 ${ }^{[95]}$ 、芳胺 ${ }^{[96]}$ 、吡 咯 ${ }^{[97]}$ 、烯丙基硅 ${ }^{[98]}$ 、烯醇硅梄 ${ }^{[98]}$ 的亲电三氟甲硫基化反 应. 对于烯丙醇的底物, 他们也同样采用该试剂, 可以 实现重排反应 ${ }^{[95]}$ (Scheme 45).

该试剂参与的反应一般都需要铜盐的催化才能发 生，其反应机理可能是该卡宾前体在铜盐作用下首先形 成卡宾中间体，随后发生重排来生成活性中间体，最后 与亲核底物发生三氟甲硫基化反应(Scheme 46).

在 2016 年, 他们课题组采用相似的策略合成了偶 氮类型的试剂，该试剂除去实现以上大部分反应外，还 可以实现碘苯的三氟甲硫基化反应，但是效率偏低 ${ }^{[99]}$ (Scheme 47).

\subsection{4 $\mathrm{N}$-三氟甲硫基二酰亚胺试剂}

该类结构的酰胺试剂目前有两种, 包括 $N$-三氟甲 硫基丁二酰亚胺以及 $N$-三氟甲硫基邻苯二甲酰亚胺. 该两种试剂的首次合成分别由 Haas 小组 ${ }^{[100]}$ 和 Munavalli 小组 ${ }^{[101]}$ 于 1996 年和 2000 年报道(Scheme 48). 


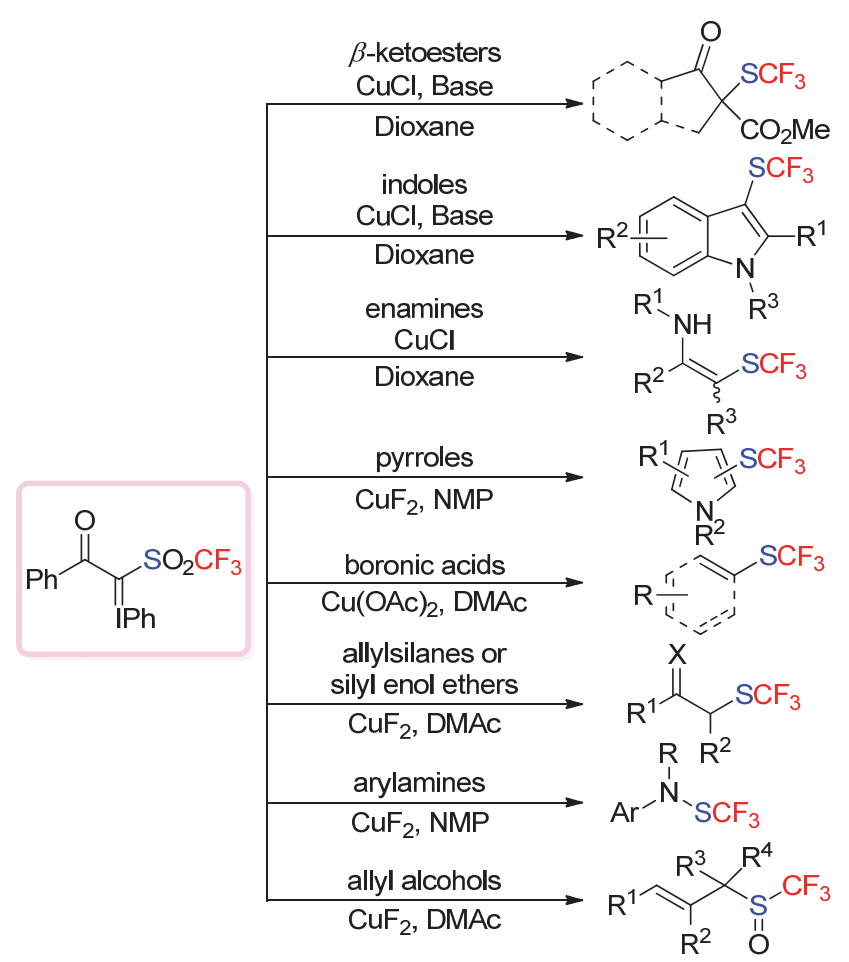

图式 45 Shibata 试剂的反应

Scheme 45 Reactions of Shibata reagent

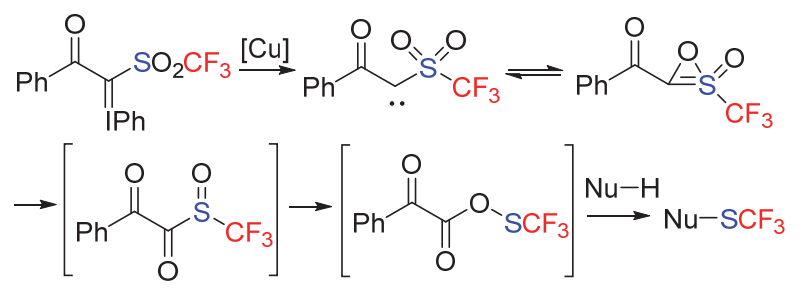

图式 46 Shibata 试剂的反应机理

Scheme 46 Proposed mechanism for $\mathrm{Cu}(\mathrm{I})$-catalyzed trifluoromethylthiolation with Shibata reagent

$$
\text { (2.5 equiv.) }
$$

图式 47 偶氮类型的 Shibata 试剂

Scheme 47 Diazo-type of Shibata reagent

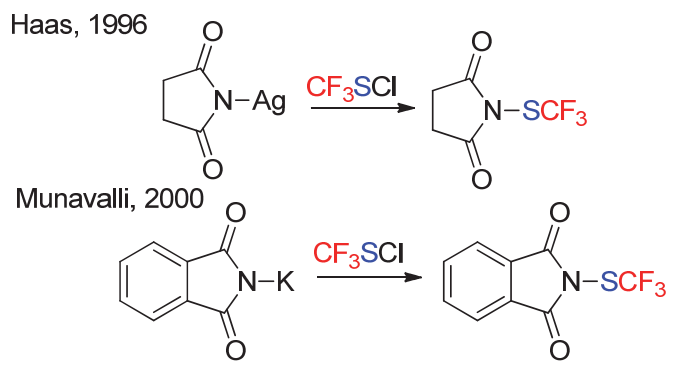

图式 48 二酰亚胺 $N-\mathrm{SCF}_{3}$ 试剂的早期制备

Scheme 48 Early preparation of $\mathrm{N}-\mathrm{SCF}_{3}$ reagent
Munavalli 小组 ${ }^{[101]}$ 首先使用 $N$-三氟甲硫基邻苯二甲 酰亚胺尝试了烯胺底物的亲电反应，发现可以以 $88 \%$ 的 收率获得相应羰基 $\alpha$ 位三氟甲硫基化反应的产物, 初步 验证了该化合物作为一种亲电三氟甲硫基化试剂的可 能性(Scheme 49). 但当他们采用该试剂尝试其他类型 亲核试剂的反应时，却没有获得成功.

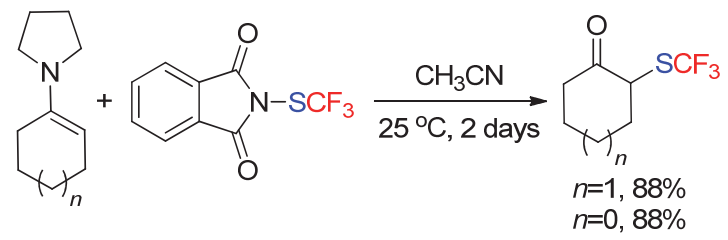

图式 49 Munavalli 小组的初步尝试

Scheme 49 Preliminary trifluoromethylthiolation attempts by Munavalli group

该类试剂在往后的十多年内，似乎已被研究人员所 “遗忘”。直到 2013 年德国的 Rueping 小组 ${ }^{[102]}$ 和上海有 机所的沈其龙小组 ${ }^{[103]}$ 几乎同时报道了该试剂的合成以 及反应, 让该试剂重新回到人们的视线. 他们在合成中 均避开了高毒性的 $\mathrm{CF}_{3} \mathrm{SCl}$, 分别采用 $\mathrm{CuSCF}_{3}$ 和 $\mathrm{AgSCF}_{3}$ 与 $N$-卤化合物发生复分解反应, 来高效、安全 地制备该试剂(Scheme 50).

\section{Rueping's Method}

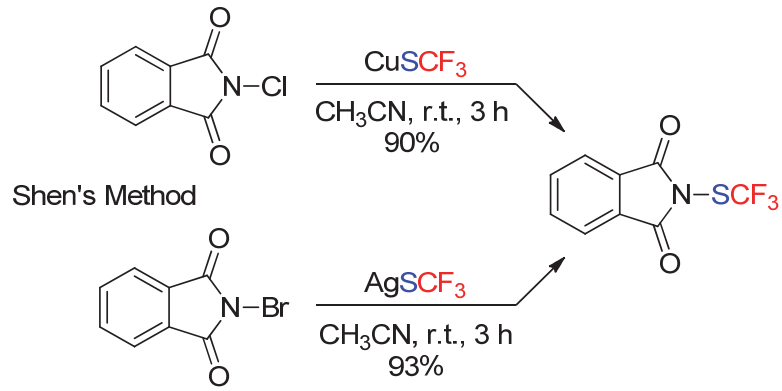

图式 $50 \mathrm{~N}$-三氟甲硫基邻苯二甲酰亚胺试剂合成方法的改良 Scheme 50 Modified preparation of $N$-(trifluoromethylthio)phthalimide by Rueping and shen group

2013 年, Rueping 小组 ${ }^{[104]}$ 使用该试剂, 首先报道了 在奎宁定作为有机碱条件下 $\beta$-酮酸酯的不对称三氟甲 硫基化反应. 该反应无论对于苯并五元、六元或者是非 苯并的 $\beta$-酮酸酯类底物都可以获得优秀的产率和对映 选择性，并且底物中的酯基取代基对对映选择性的影响 不大. 随后，他们又在金鸡纳碱(DHQD) ${ }_{2} \mathrm{Pyr}$ 作为有机 碱的条件下实现了 2-吲哚酮底物的不对称三氟甲硫基 化反应 ${ }^{[105]}$ (Scheme 51). 该体系与沈其龙小组的反应体 系形成了互补，当底物 3 位上的取代基是芳基时可以获 得优秀的产率和对映选择性.

2014 年, Rueping 小组 ${ }^{[102]}$ 和沈其龙小组 ${ }^{[103]}$ 几乎同 时报道了嗍酸与该试剂在铜催化下的偶联反应, 反应条 件非常相似, 有着优秀的底物兼容性(Scheme 52). 采用 


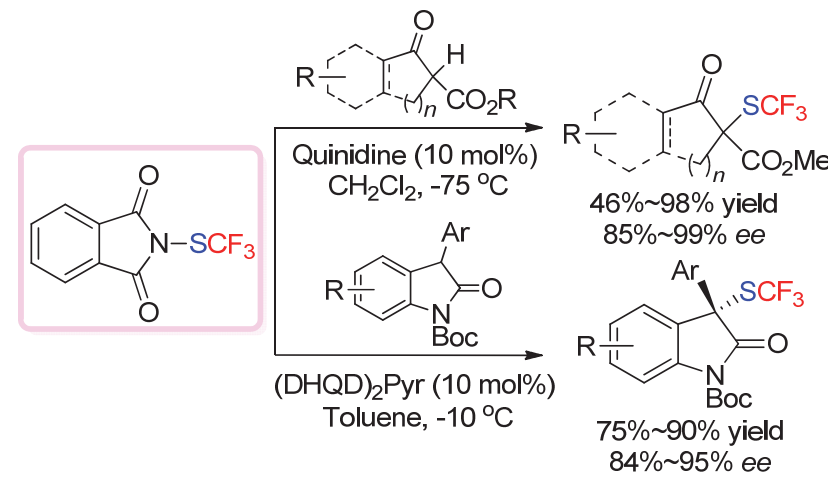

图式 $51 \mathrm{~N}$-三氟甲硫基邻苯二甲酰亚胺试剂参与的不对称反应

Scheme 51 Asymmetric trifluoromethylthiolation using $N$-(trifluoromethylthio)phthalimide

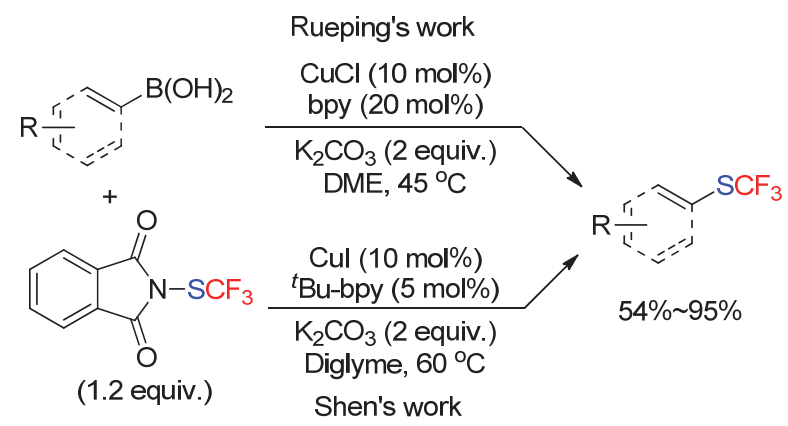

图式 $52 \mathrm{~N}$-三氟甲硫基邻苯二甲酰亚胺试剂与嗍酸的偶联反应

Scheme 52 Cross-coupling reaction between boronic acids and $\mathrm{N}$-(trifluoromethylthio)phthalimide

该试剂, Rueping 小组 ${ }^{[02]}$ 也实现了铜催化下炔烃的偶联 反应. 另外, 通过他们后续的研究发现, 采用该试剂, 可以在使用三氟甲苯作为反应溶剂的条件下，实现硫 醇、硫酚以及胺类底物的三氟甲硫基化反应 ${ }^{[106]}$ (Scheme $53)$.

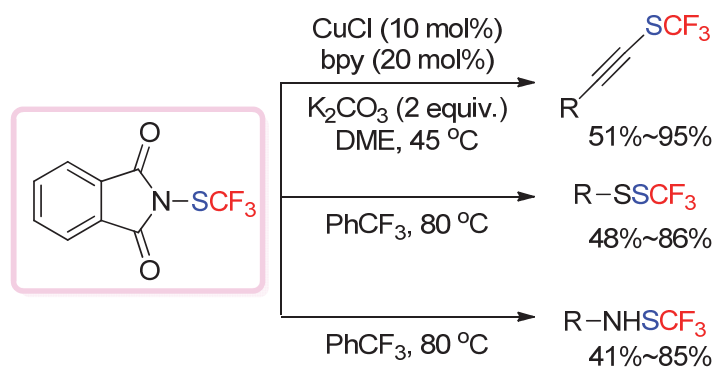

图式 53 Rueping 小组其它的工作

Scheme 53 Other works by Rueping group

2015 年，南方科技大学的汪珺小组 ${ }^{[107]}$ 采用该试剂 实现了活化烯烃底物的双官能团化反应. 同时他们发 现, 采用硝基取代的试剂, 可以进一步提高试剂的反应 活性, 从而可以进一步提高产率(Scheme 54).

Glorius 小组 ${ }^{[108]}$ 采用该试剂也做出了漂亮的工作. 2015 年, 他们采用 $\mathrm{NaCl}$ 为添加剂, 实现了吡咯、吲哚、 氮杂吲哚等底物的三氟甲硫基化反应(Scheme 55). 他 们认为, 在该反应体系中, 加入的 $\mathrm{NaCl}$ 可能与试剂反
应现场生成了高活性的 $\mathrm{CF}_{3} \mathrm{SCl}$ ，但是他们并未检测到 该中间体的存在，因此 $\mathrm{NaCl}$ 所起的作用还有待考证. 此外，他们采用该试剂在光催化条件下也实现了一系列 自由基类型的三氟甲硫基化反应，我们将在后续进行介 绍.<smiles>[R][R]([Y])=CC(=O)N1C(=O)c2ccc([X])cc2C1=O</smiles>

图式 54 碱促进下不饱和羰基化合物的胺化三氟甲硫基化反应 Scheme 54 DABCO-promoted aminotrifluoromethylthiolation of $\alpha, \beta$-unsaturated carbonyl compounds

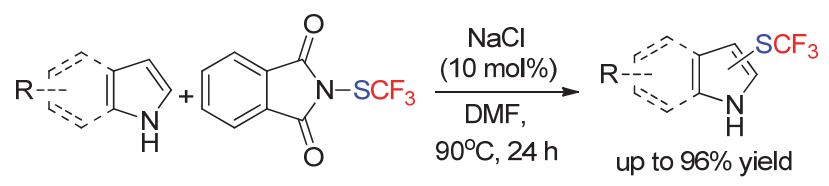

图式 $55 \mathrm{NaCl}$ 催化下含氮杂环的三氟甲硫基化反应

Scheme $55 \mathrm{NaCl}$-catalyzed trifluoromethylthiolation of $\mathrm{N}$-heteroarenes

$N$-三氟甲硫基丁二酰亚胺试剂随后也由沈其龙小 组 ${ }^{[109]}$ 采用相似的策略合成. 他们采用该试剂, 首次实 现了吡啶导向的芳烃 $\mathrm{C}-\mathrm{H}$ 键三氟甲硫基化反应 (Scheme 56).

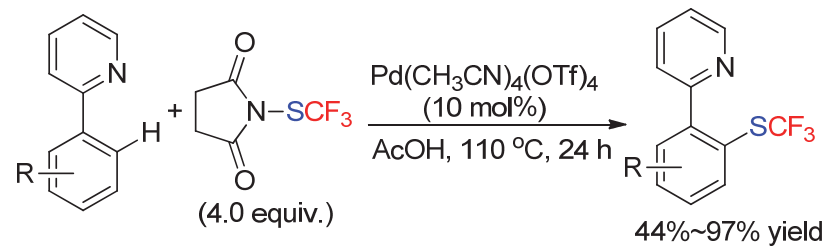

图式 $56 \mathrm{Pd}$ 催化芳基 $\mathrm{C}-\mathrm{H}$ 三氟甲硫基化反应

Scheme 56 Palladium-catalyzed trifluoromethylthiolation of Aryl C-H bonds

采用该丁二酰亚胺试剂, 北京理工大学的杜大明小 组 ${ }^{[110]}$ 在双方酰化氨基酸配体的催化下，实现了一锅法 的不对称三氟甲硫基化和 Michael/aldol 串联反应，以优 秀的对映选择性和良好的非对映选择性得到了串联环 化的产物(Scheme 57).

\subsubsection{Shen 试剂}

在 2014 年, 上海有机所沈其龙小组 ${ }^{[11]}$ 在发展 $N$-三 氟甲硫基邻苯二甲酰亚胺试剂的基础上，进一步报道合 成了基于糖精骨架的亲电三氟甲硫基化试剂(Scheme 58)。该试剂的合成同样极为方便，且有着更为出色的 反应性能. 通过他们小组的研究发现，采用该试剂可以 在温和条件下实现醇、胺、硫醇、硫酚、富电子芳烃、 羰基 $\alpha$ 位 $\mathrm{C}-\mathrm{H}$ 键、酮酸酯、炔烃等一系列底物的三氟 甲硫基化反应. 而当采用 $\mathrm{TMSCl}$ 作为 Lewis Acid 时, 他 们也报道了首例分子内羧酸、酰胺与双键的双官能团化 三氟甲硫基化反应 ${ }^{[112]}$ (Scheme 59). 

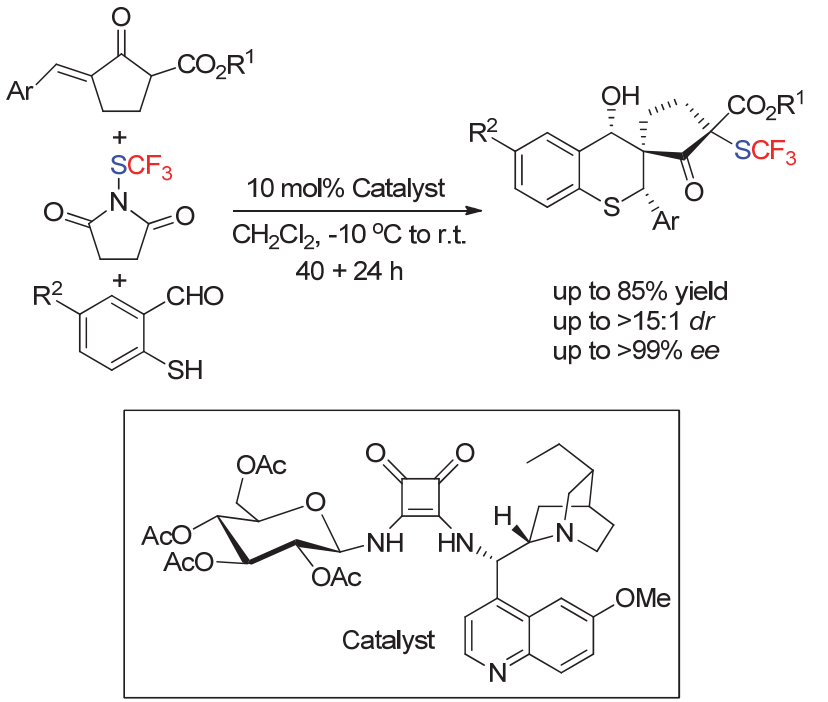

图式 57 双方酰化氨基酸配体催化下不对称三氟甲硫基化反应 Scheme 57 Enantioselective squaramide-catalyzed trifluoromethylthiolation-sulfur-Michael/adol cascade reaction

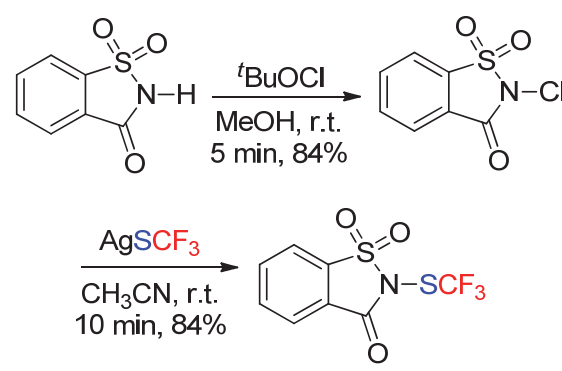

图式 58 Shen 试剂的合成

Scheme 58 Preparation of $N$-trifluoromethylthiosaccharin

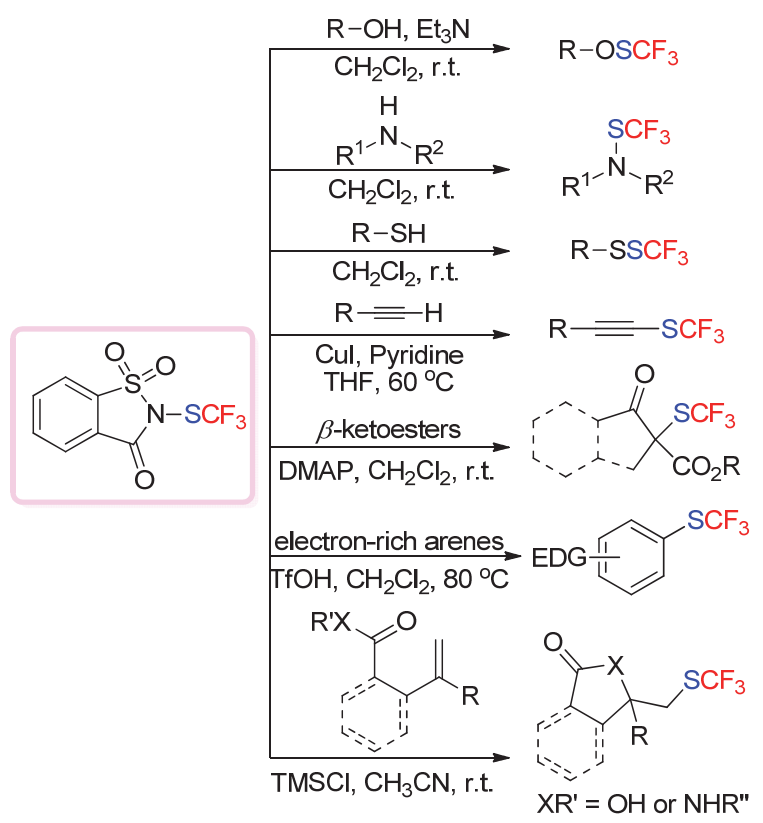

图式 59 Shen 试剂的反应

Scheme 59 Reactions of $N$-trifluoromethylthiosaccharin
除此之外，其他小组采用糖精试剂，也实现了其他 转化. 如 2015 年大连化物所的李兴伟小组 ${ }^{[13]}$ 采用 $\mathrm{FeCl}_{3}$ 或 $\mathrm{AuCl}_{3}$, 实现了富电子芳烃底物的三氟甲硫基化反应. 另外，他们采用官能团导向的策略实现了 Rh(III)催化吲 哚 2 位 $\mathrm{C}-\mathrm{H}$ 键的三氟甲硫基化反应 ${ }^{[114]}$ (Scheme 60).<smiles>O=C1c2ccccc2S(=O)(=O)N1S(=O)(=O)N1C(=O)c2ccccc2S1(=O)=O</smiles>

图式 60 李兴伟小组的工作

Scheme 60 Works by Li group

Shibata 小组与 Cahard 小组 ${ }^{[15]}$ 合作, 采用该试剂与 烯丙醇底物反应, 发现可以经过重排反应生成烯丙基三 氟亚砜的产物(Scheme 61). 在该反应中, 二级烯丙醇可 以获得热力学稳定的反式选择性的产物. 而对于一级、 三级烯丙醇类底物, 作者并没有进行相关地考察.

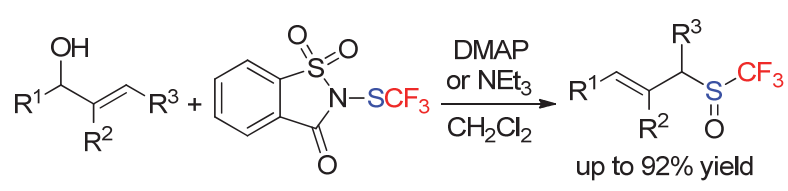

图式 61 烯丙醇与 Shen 试剂在碱促进下的重排反应

Scheme 61 Reactions between allylic alcohols and Shen reagent to form trifluoromethyl sulfoxides via [2,3]-Sigmatropic rearrangement

中山大学的赵晓丹小组 ${ }^{[116]}$ 使用二芳基硒作为催化 剂，在三氟磺酸作为添加剂的条件下，实现了苯乙烯以 及烯烃衍生物的双官能团化三氟甲硫基化反应. 他们认 为, 二芳基硒与糖精类型的试剂在三氟甲磺酸的存在下 可以生成活性的 $\left[\mathrm{Se}^{-} \mathrm{SCF}_{3}\right]$ 中间体，接受烯烃的进攻后 形成的阳离子可进一步被体系中的亲核试剂捕获，从而 生成双官能团化产物。随后, 他们采用相似的策略, 发 现对于炔烃底物，同样可以实现相应的双官能团化反 应，并且有着优秀的顺反选择性 ${ }^{[117]}$. 他们也对该体系 下的手性硫醚、硒醚催化剂进行了考察. 而通过一系列 的篮选发现，狮胺醇衍生的手性硫醚催化剂，可以很好 地实现烯烃内酯化三氟甲硫基化反应. 当采用糖精亲电 三氟甲硫基化试剂在此条件下反应时，可以获得 $92 \%$ 的 收率和 $83 \%$ 的对映选择性 ${ }^{[18]}$ (Scheme 62).

北京大学叶新山小组 ${ }^{[19]}$ 发现, 使用烯糖与糖精试 剂在 TMSCl 作为 Lewis Acid 的条件下反应, 会生成氯 化三氟甲硫基化的双官能团化产物，该产物在 DBU 作 为碱的条件下，可以顺利地发生消除反应，从而实现双 


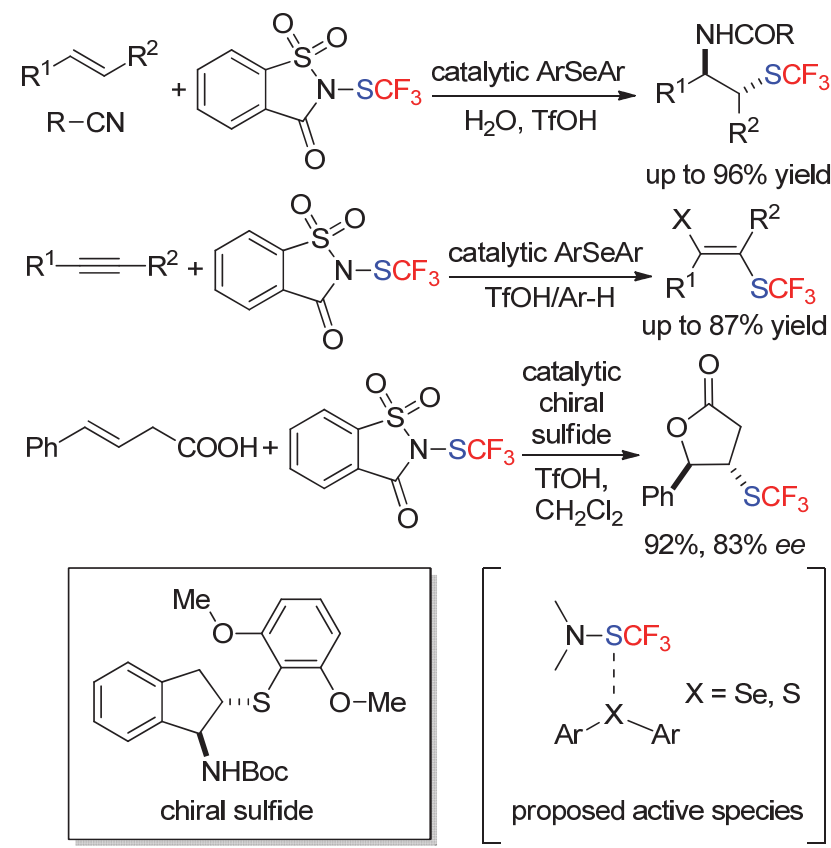

图式 62 赵晓丹小组的工作

Scheme 62 Zhao's works

键 $\mathrm{C}-\mathrm{H}$ 上的三氟甲硫基化反应(Scheme 63). 作者考察 多种烯糖类底物, 发现都可以以优秀的收率得到相应的 产物.

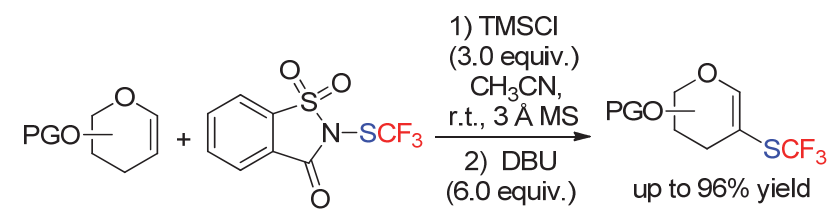

图式 63 烯糖 $\mathrm{C}-\mathrm{H}$ 键三氟甲硫基化反应

Scheme 63 2-Trifluoromethylthiolation of glycals

湖北科技学院的吴鸣虎和孙绍发小组 ${ }^{[120]}$ 利用烯胺 中间体的亲核性, 通过对二级胺的篮选, 发现吡咯烷可 以有效地催化醛底物和糖精试剂的反应. 作者通过硼氢 化钠现场对羰基进行还原, 实现了一级醇 $\alpha$ 位三氟甲硫 基取代产物的构建(Scheme 64).

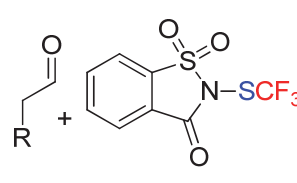

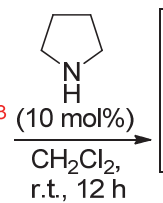<smiles>[R]C=CN1CCCC1</smiles>

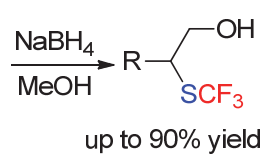

图式 64 醛 $\alpha$ 位三氟甲硫基化反应

Scheme $64 \alpha$-Trifluoromethylthiolation of aldehydes

Glorius 小组 ${ }^{[121]}$ 采用之前提到过的 $\mathrm{NaCl}$ 活化的策 略, 采用糖精试剂, 实现了呋喃 2 位 $\mathrm{C}-\mathrm{H}$ 键的三氟甲 硫基化反应(Scheme 65). 作者认为该反应可能也是生 成了高活性的 $\mathrm{CF}_{3} \mathrm{SCl}$ 中间体. 但是该反应采用 $\mathrm{N}$-三氟 甲硫基邻苯二甲酰亚胺却不能反应，因此反应体系的真 正活性中间体是否是 $\mathrm{CF}_{3} \mathrm{SCl}$ 还只是个推测.

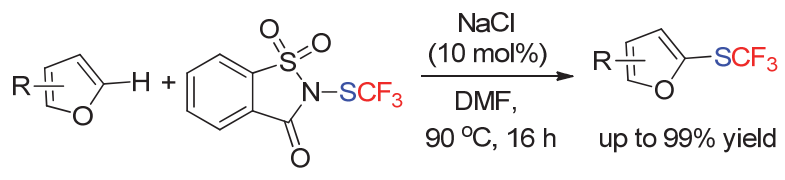

图式 65 呋喃衍生物的三氟甲硫基化反应

Scheme 65 NaCl-Catalyzed trifluoromethylthiolation of furans

山东大学徐政虎小组 ${ }^{[122]}$ 首先通过三组分的 Click 反应构建了 5 -锡基三氮唑化合物，随后在 $\mathrm{AlCl}_{3}$ 作为 Lewis Acid 的条件下与糖精试剂反应，两步构建了 5-三 氟甲硫基取代的三氮唑化合物(Scheme 66).

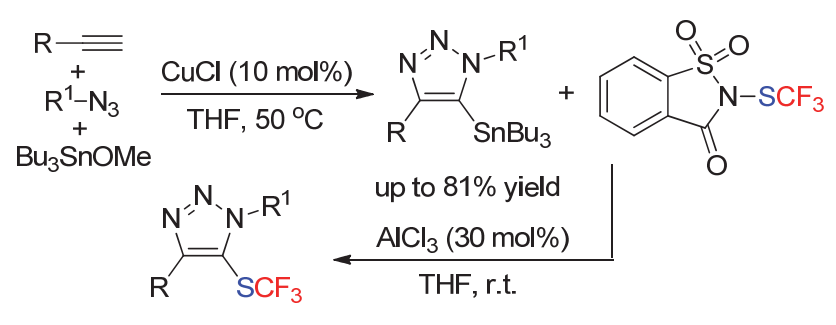

up to $84 \%$ yield

图式 66 5-锡基三氮唑的三氟甲硫基化反应

Scheme 66 5-Stannyl triazole towards trifluoromethylthiotriazoles

\subsection{6 $\mathrm{N}$-三氟甲硫基双苯磺酰亚胺试剂}

2016 年上海有机所的吕龙和沈其龙小组 ${ }^{[123]}$ 进一步 报道了基于双苯磺酰亚胺类型的新型亲电三氟甲硫基 试剂(Scheme 67). 他们对该试剂的反应活性进行了细 致地研究，发现并支持了该试剂高的亲电三氟甲硫基活 性.

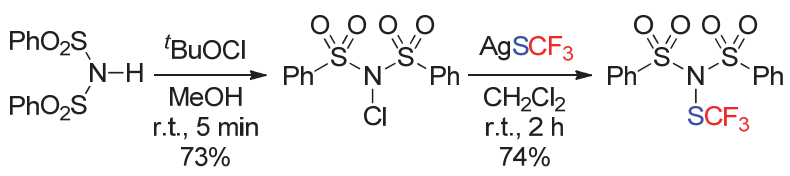

图式 $67 \mathrm{~N}$-三氟甲硫基双苯磺酰亚胺亲电三氟甲硫基试剂的制备 Scheme 67 Preparation of $N$-trifluoromethylthio-dibenzenesulfonimide

他们与南开大学薛晓松小组合作, 对该试剂的亲电 三氟甲硫基化活性进行了理论计算 ${ }^{[123,124]}$ (Scheme 68). 计算结果以 $\mathrm{Tt}^{+} \mathrm{DA}$, 即给出三氟甲硫基阳离子的能力给 出. 结果表明，该试剂在目前具有最高的亲电三氟甲硫 基化活性，其活性甚至高过了 $\mathrm{CF}_{3} \mathrm{SCl}$. 基于该理论计算 的结果，他们对该试剂的反应活性进行了较为系统的考 察.

采用该试剂, 他们首先实现了无添加剂存在条件下 富电子芳烃、杂芳烃底物的三氟甲硫基化反应. 随后, 他们使用 2-荎酚衍生物发现了去芳构化反应，并在三氟 磺酸钪和双恶唑啉配体的催化下, 实现了首例去芳构化 三氟甲硫基化反应(Scheme 69).

此外，采用该试剂，作者发现，同样无需添加剂的 活化，便可以实现苯乙烯衍生物 $\mathrm{C}-\mathrm{H}$ 键反式选择性的 


\begin{tabular}{lllll} 
Reagents & $\begin{array}{c}\mathrm{Tt}+\mathrm{DA} \text { in } \mathrm{CH}_{2} \mathrm{Cl}_{2} \\
\left(\mathrm{kcall}-\mathrm{mol}^{-1}\right)\end{array}$ & Reagents & $\begin{array}{c}\mathrm{Tt}^{+} \mathrm{DA} \text { in } \mathrm{CH}_{2} \mathrm{Cl}_{2} \\
\left(\mathrm{kcal} \cdot \mathrm{mol}^{-1}\right)\end{array}$ \\
\hline
\end{tabular}

图式 68 亲电三氟甲硫基试剂理论计算结果

Scheme $68 \mathrm{Tt}^{+} \mathrm{DA}$ of electrophilic trifluoromethylthiolating reagents

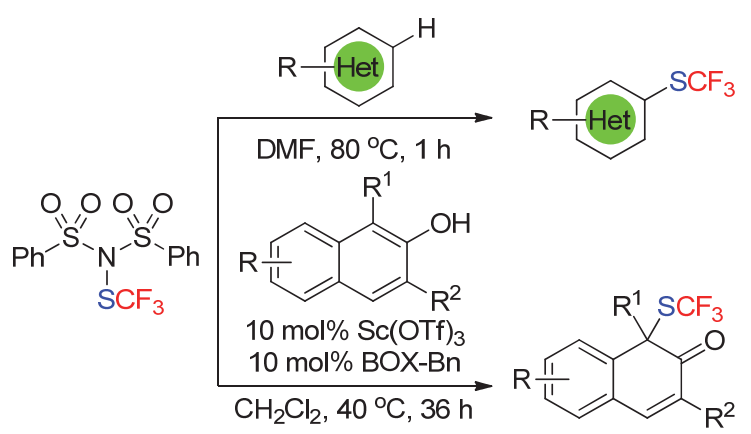

图式 $69 \mathrm{~N}$-三氟甲硫基双苯磺酰亚胺的反应

Scheme 69 Reactions between heteroarenes and naphthol derivatives with $N$-trifluoromethylthio-dibenzenesulfonimide

三氟甲硫基化反应. 作者对该反应的机理进行了研究, 发现该过程经历了碳正离子过程. 苯乙烯衍生物在常温 下会和该试剂反应从而生成碳正离子中间体或者 DMF 进攻碳正离子中间体所形成的亚胺离子中间体. 中间体 在加热条件下发生会发生消除反应，从而生成烯烃产物 (Scheme 70).

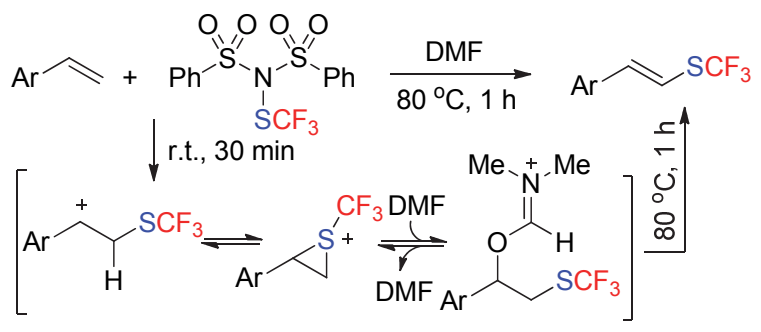

图式 70 苯乙烯衍生物 $\mathrm{C}-\mathrm{H}$ 键三氟甲硫基化反应及机理

Scheme 70 Reaction and mechanism for direct trifluoromethylthiolation of styrene derivatives

随后，作者在该机理研究的基础上，实现了一系列 双官能团化反应(Scheme 71). 作者通过改变反应的溶 剂, 实现了烯烃衍生物的甲酰氧化、乙酰氧化、羊弪化以 及胺化三氟甲硫基化反应.

他们同时也尝试了一系列碳亲核试剂以及杂原子 亲核试剂的反应, 发现都可以以优秀的收率得到相应的 产物(Scheme 72).

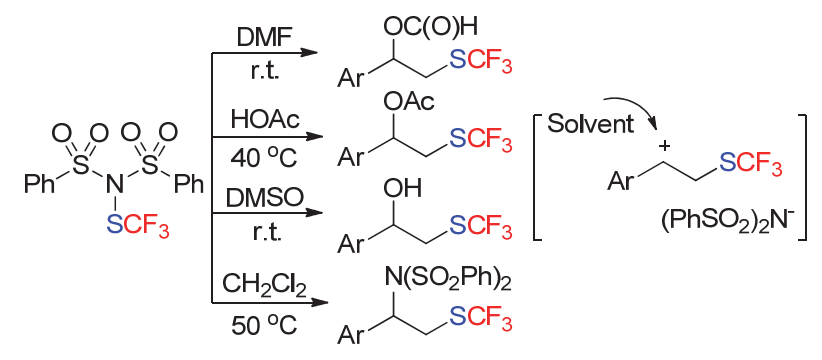

图式 71 烯烃衍生物的双官能团化三氟甲硫基化反应 Scheme 71 Difunctionalized trifluoromethylthiolation

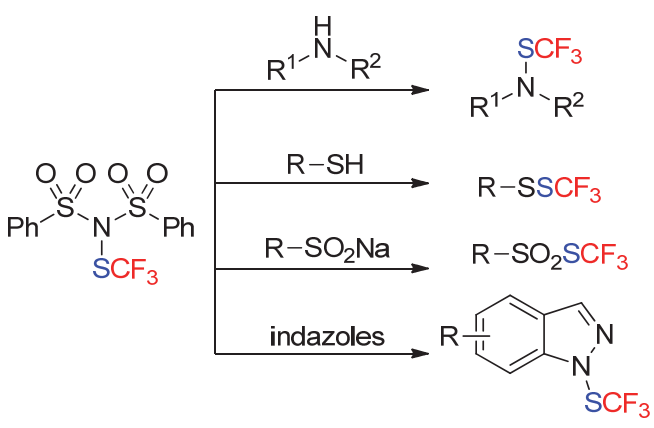

图式 72 杂原子亲核试剂的三氟甲硫基化反应

Scheme 72 Trifluoromethylthiolation of heteroatom-nucleophiles

前面我们提到中山大学的赵晓丹小组 ${ }^{[18]}$ 实现了分 子内的内酯化反应(Scheme 73). 他们在该工作中也对 该双苯磺酰亚胺试剂进行了合成. 相比较于糖精结构的 试剂, 该试剂可以获得更优秀的对映选择性. 但是在他 们的工作中，对于该试剂的分离纯化问题没有得到很好 地解决.

$$
\overbrace{\mathrm{COOH}+\mathrm{Ph}^{-}}^{\text {up to } 93 \% \text { yield }}
$$

图式 73 手性硫醚催化下的不对称内酯化三氟甲硫基化反应

Scheme 73 Enantioselective trifluoromethylthiolating lactonization catalyzed by an indane-based chiral sulfide

\subsection{7 手性亲电三氟甲硫基试剂}

在 2017 年年初, 上海有机所的沈其龙小组 ${ }^{[125]}$ 报道 了第一例手性骨架的亲电三氟甲硫基试剂. 他们从樟脑 磺酰胺出发, 与次氯酸叔丁酯生成 $N-\mathrm{Cl}$ 前体后与 $\mathrm{AgSCF}_{3}$ 发生复分解反应, 可以以优秀的收率得到相应 的手性亲电三氟甲硫基试剂(Scheme 74). 采用相同的 方法，他们也合成了基于恶唑烷酩骨架的手性亲电三氟 甲硫基试剂.

采用该类手性试剂，作者发现，使用二甲氧基取代 的手性磺酰胺试剂, 可以很好地实现 $\beta$-酮酸酯底物、2吲哚酮以及苯并呋喃酮底物的不对称三氟甲硫基化反 应，实现试剂手性向底物手性的传递 ${ }^{[125]}$. 并且对于该 类反应, 只需要加入催化量的碱, 便可以顺利地实现转 
化(Scheme 75).

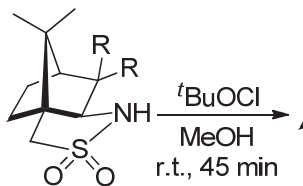

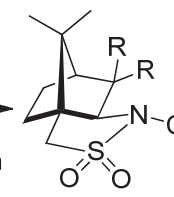
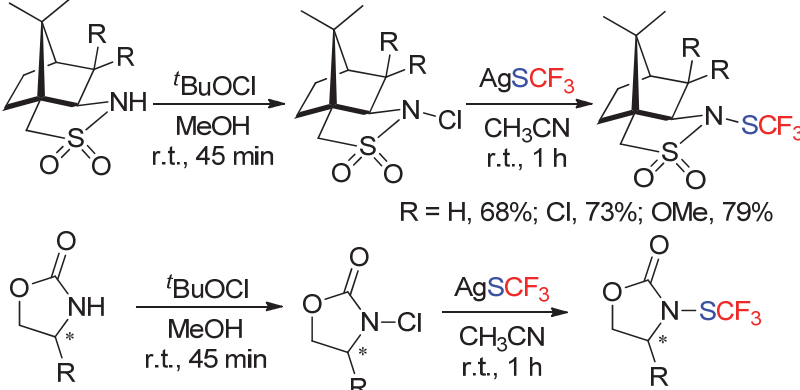

$\underbrace{O}_{V^{N}}$

$\mathrm{R}=\mathrm{H}, 68 \% ; \mathrm{Cl}, 73 \% ; \mathrm{OMe}, 79 \%$

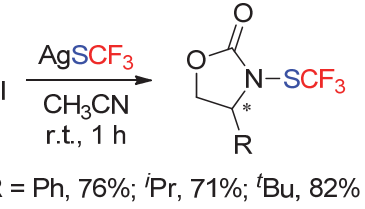

图式 74 手性亲电三氟甲硫基试剂的合成

Scheme 74 Preparation of optically pure trifluoromethylthiolation reagents

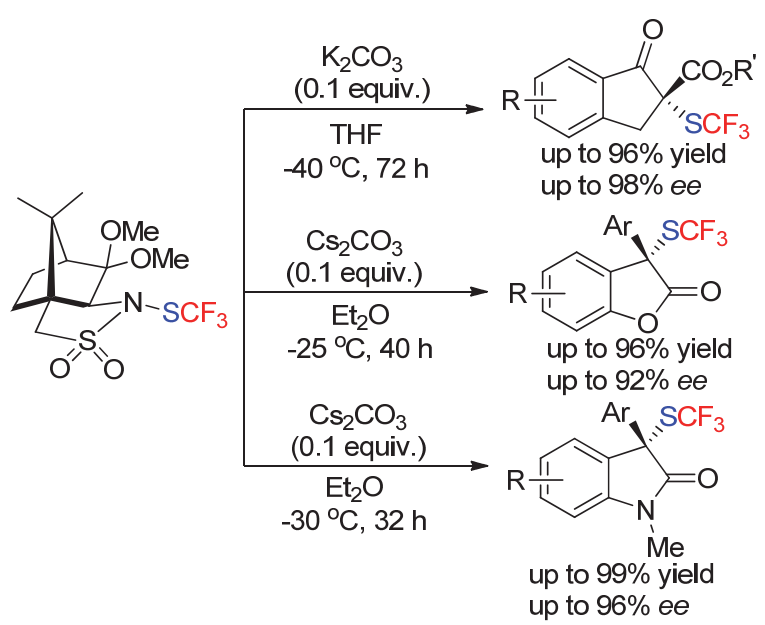

图式 $75 \beta$-酮酸酯、苯并呋喃酮及氧化吲哚底物的不对称三氟甲硫基

化反应

Scheme 75 Trifluoromethylthiolation of $\beta$-ketoesters, oxindoles and benzofuranones

\subsection{8 现场生成亲电三氟甲硫基试剂}

利用稳定且易于制备的亲电三氟甲硫基试剂，可以 高效地在分子中引入三氟甲硫基团. 而通过现场生成亲 电试剂的策略，则无需对亲电试剂进行预先制备和分 离, 进一步简化了反应操作.

2014 年, 南方科技大学的谭斌和刘心元小组 ${ }^{[126]}$ 通 过考察一系列金鸡钠碱催化剂, 实现了 3-芳基-2-吲哚 酮底物的不对称三氟甲硫基化(Scheme 76). 他们通过 $\mathrm{AgSCF}_{3}$ 和 $\mathrm{TCCA}$ 现场生成亲电三氟甲硫基物种的策略 实现了该步转化. 而在他们初步的机理研究中, 发现 $\mathrm{CF}_{3} \mathrm{SSCF}_{3}$ 是该反应的活性物种之一, 此外, 可能存在的 其他亲电三氟甲硫基物种尚未被确定.

上海药物所的杨春皓小组 ${ }^{[27]}$ 采用同样的策略, 在 温和的条件下对不同取代基取代的色酮进行了合成 (Scheme 77). 该反应的机理可能是该推拉电子的底物 首先发生分子内的迈克尔加成反应, 随后生成的中间体 与现场产生的亲电三氟甲硫基物种反应，最后脱除二甲
基胺生成最终产物.

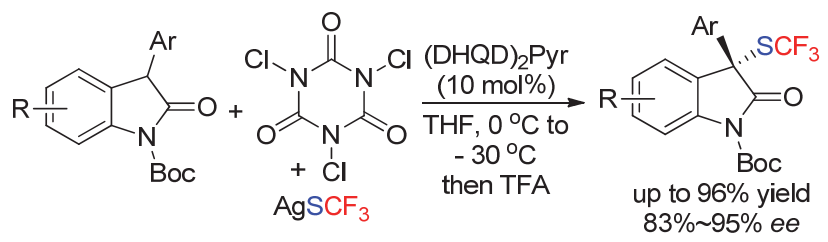

图式 $76 \mathrm{AgSCF}_{3} / \mathrm{TCCA}$ 现场生成亲电三氟甲硫基试剂实现氧化吲哚 的不对称三氟甲硫基化反应

Scheme 76 In situ generation of electrophilic trifluoromethylthio reagents for enantioselective trifluoromethylthiolation of oxindoles

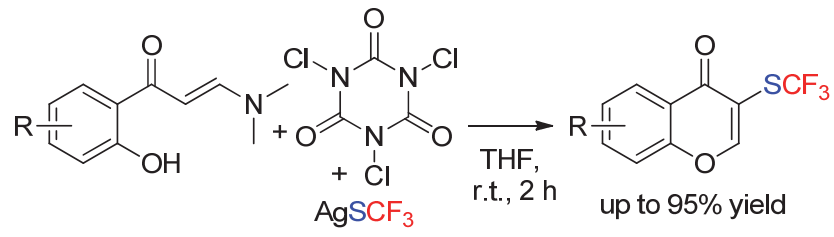

图式 $77 \mathrm{AgSCF}_{3} / \mathrm{TCCA}$ 现场生成亲电三氟甲硫基试剂实现色酮的合 成

Scheme 77 In situ generation of electrophilic trifluoromethylthio reagents for the synthesis of 3-((trifluoromethyl)thio)-4H-chromen-4-on

卿风领挧组 ${ }^{[128]}$ 从未端炔烃出发, 使用 $\mathrm{AgSCF}_{3}$ 和 NCS，在磷酸钾作碱的条件下，实现了末端炔烃的三氟 甲硫基化反应(Scheme 78). 有意思的是，对于该反应， 先预先合成亲电三氟甲硫基化试剂，反应是不能发生 的. 因此, 反应体系中存在的银盐可能对该反应起到了 关键性的作用!

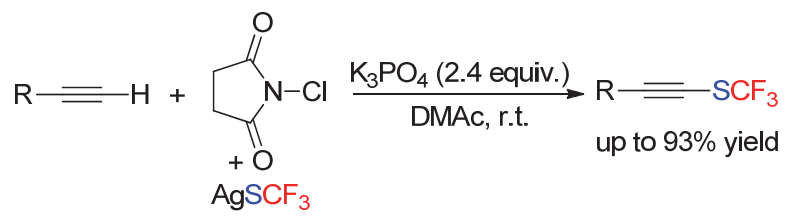

图式 $78 \mathrm{AgSCF}_{3} / \mathrm{NCS}$ 下实现末端炔烃的三氟甲硫基化反应 Scheme 78 Trifluoromethylthiolation of terminal alkynes using $\mathrm{AgSCF}_{3}$ and NCS

而在之前我们所提到的亲电三氟甲硫基化试剂的 制备大多都是采用亲核性的三氟甲硫基源来完成的. 2015 年，南京理工大学的易文斌与 UMass Boston 的张 玮课题组 ${ }^{[129]}$ 合作, 使用廉价的三氟亚磺酸钠 $\left(\mathrm{CF}_{3} \mathrm{SO}_{2} \mathrm{Na}\right.$, Langlois reagent, 常被用来作为三氟甲基自 由基试剂)作为三氟甲硫基源, 在亚磷酸酯作为还原剂, DMSO 与 $\mathrm{CuCl}$ 作为氧化剂和促进剂的条件下, 实现了 吲哚、吡咯以及富电子烯胺类底物的直接三氟甲硫基化 反应(Scheme 79). 作者对该反应的机理进行了初步的 考察，通过氟谱跟踪和质谱检测，作者认为，在该反应 中, 首先是三氟亚磺酸钠被亚磷酸酯还原生成活性的 $\mathrm{CF}_{3} \mathrm{~S}(=\mathrm{O}) \mathrm{H}$, 随后发生重排生成 $\mathrm{CF}_{3} \mathrm{~S}-\mathrm{OH}$. 该物种可以 直接作为亲电试剂与富电子底物发生反应或者是在 $\mathrm{DMSO}$ 与 $\mathrm{CuCl}$ 的存在下生成 $\mathrm{CF}_{3} \mathrm{SSCF}_{3}$, 从而进一步与 底物发生反应. 


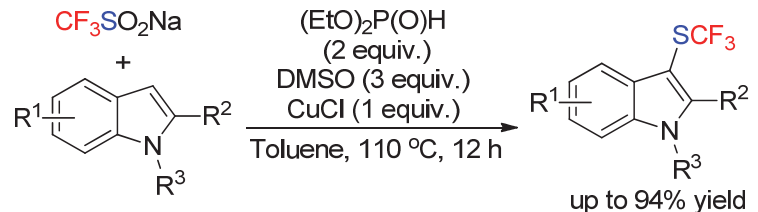

Proposed mechanism

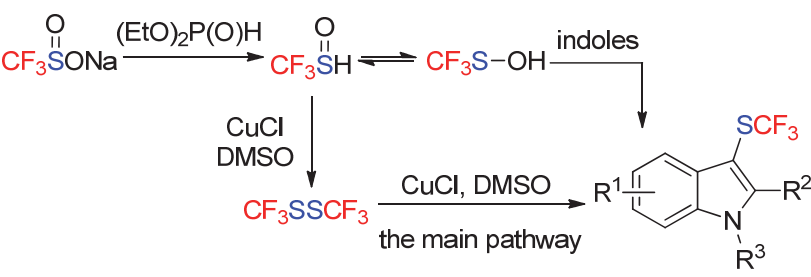

图式 $79 \mathrm{CF}_{3} \mathrm{SO}_{2} \mathrm{Na}$ 作为三氟甲硫基源实现吲哚的三氟甲硫基化反应 Scheme 79 Direct trifluoromethylthiolation of indoles with $\mathrm{CF}_{3} \mathrm{SO}_{2} \mathrm{Na}$

此前我们也提到, Vicic 小组 ${ }^{[35]}$ 使用 $\mathrm{PPh}_{3}$ 与 $\mathrm{CF}_{3} \mathrm{SO}_{2} \mathrm{Na}$ 反应, 同样观测到了 $\mathrm{CF}_{3} \mathrm{SSCF}_{3}$ 的生成. 随后, 他们在反应体系中加入 $\mathrm{CuCl}$, 可以以 $80 \%$ 的氟谱收率, 观测到 $\mathrm{CuSCF}_{3}$ 的生成.

在 2016 年, Shibata 小组与 Cahard 小组 ${ }^{[130]}$ 合作, 使 用 $\mathrm{CF}_{3} \mathrm{SO}_{2} \mathrm{Cl}$ 作为三氟甲硫基源, 在 $\mathrm{PMe}_{3}$ 作为还原剂的 条件下实现了吲哚、吡咯、烯胺等富电子底物的亲电三 氟甲硫基化反应. 他们对反应机理进行了初步的考察, 发现体系中的 $\mathrm{CF}_{3} \mathrm{SCl}$ 是该反应的活性物种, 而 $\mathrm{CF}_{3} \mathrm{SSCF}_{3}$ 不具有反应活性. 天津科技大学的赵霞小 组 ${ }^{[131]}$ 使用相似的策略, 发现在体系中加入催化量的碘 化钠可以更有效地促进该反应的发生. 易文斌小组 ${ }^{[132]}$ 采用亚磷酸酯为还原剂, 在他们已有工作的基础上, 也 可以实现吲哚底物的三氟甲硫基化反应. 并且在此条件 下，吡咯衍生物、苯甲醚衍生物以及硫酚衍生物也可以 很好地实现三氟甲硫基化转化(Scheme 80).

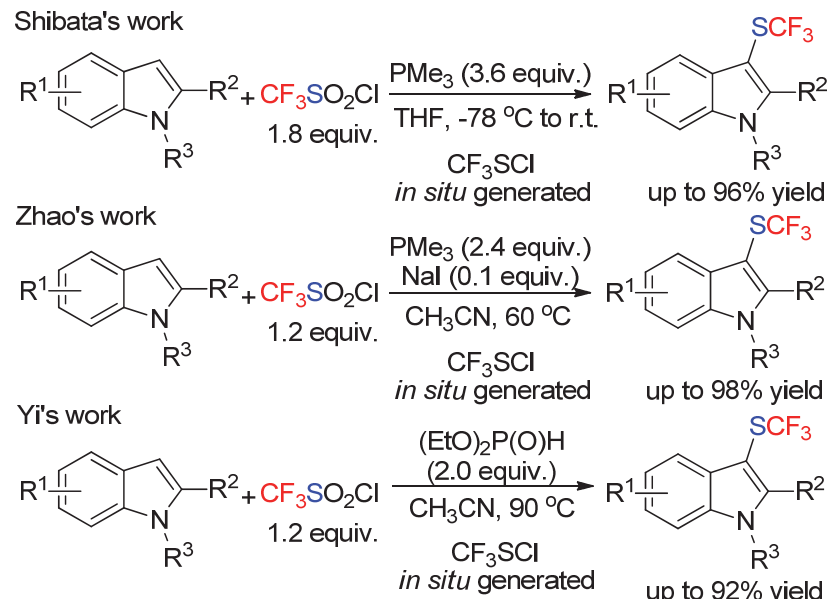

图式 $80 \mathrm{CF}_{3} \mathrm{SO}_{2} \mathrm{Cl}$ 作为三氟甲硫基源实现吲哚三氟甲硫基化反应 Scheme 80 Direct trifluoromethylthiolation of indoles with $\mathrm{CF}_{3} \mathrm{SO}_{2} \mathrm{Cl}$

南京理工大学的蔡春小组 ${ }^{[133]}$ 使用 $\mathrm{CF}_{3} \mathrm{SO}_{2} \mathrm{Na}$ 作为 三氟甲硫基源, $\mathrm{PPh}_{3}$ 作为还原剂, $N$-氯代邻苯二甲酰亚 胺作为氯源, 通过现场生成 $\mathrm{CF}_{3} \mathrm{SCl}$ 的策略, 同样实现了
吲哚、吡咯、烯胺等富电子底物的三氟甲硫基化反应 (Scheme 81).

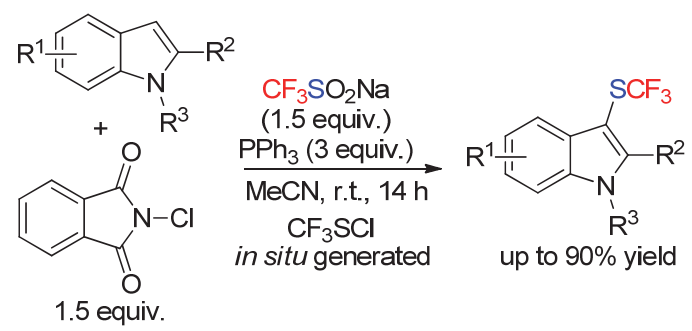

图式 $81 \quad \mathrm{CF}_{3} \mathrm{SO}_{2} \mathrm{Na}$ 作为三氟甲硫基源实现吲哚三氟甲硫基化反应 Scheme 81 Direct trifluoromethylthiolation of indoles with $\mathrm{CF}_{3} \mathrm{SO}_{2} \mathrm{Na}$

\subsection{9 亲电三氟甲硫基试剂的理论计算结果}

在 2016 年，南开大学薛小松小组 ${ }^{[123,124]}$ 对已有的亲 电三氟甲硫基试剂的亲电三氟甲硫基化活性进行了理 论计算. 他们采用阴离子交换三氟甲硫基正离子的模 型，在二氯甲烷作为反应溶剂的条件下，对 46 种亲电三 氟甲硫基试剂的亲电活性进行了理论计算(Scheme 82).

他们以 $\mathrm{CF}_{3} \mathrm{COOSCF}_{3}$ 为基准, 以给出三氟甲硫基正 离子的能力 $\left(\mathrm{Tt}^{+} \mathrm{DA}\right)$ 为指标, 对已有的亲电试剂的活性 进行了排序. 理论计算结果表明，该类试剂的反应活性 与试剂所对应的酸根阴离子的共轭酸的 $\mathrm{p} K_{\mathrm{a}}$ 有着很好的 相关性，共轭酸的 $\mathrm{p} K_{\mathrm{a}}$ 越低，其亲电三氟甲硫基化活性 越高. 如邻苯二甲酰亚胺、糖精及双磺酰亚胺的 $\mathrm{p} K_{\mathrm{a}}$ 分 别为 $10.06,1.8$ 和 1.45 , 其所对应的试剂的 $\mathrm{Tt}^{+} \mathrm{DA}$ 值为 33.0, 17.9 和 9.8. 该 9.8 的 $\mathrm{Tt}^{+} \mathrm{DA}$ 值甚至高过了高活性 的 $\mathrm{CF}_{3} \mathrm{SCl}\left(\mathrm{Tt}^{+} \mathrm{DA}\right.$ 值为 11.1), 因而也说明, 基于双苯磺 酰亚胺的亲电三氟甲硫基试剂在目前已知的亲电试剂 中有着最高的反应活性.

\subsubsection{0 其他类型的亲电三氟甲硫基化反应}

前面我们提到, Billard 小组 ${ }^{[64]}$ 利用 DAST 和 $\mathrm{TMSCF}_{3}$ 合成了其苯胺骨架的亲电三氟甲硫基试剂，该 反应的中间体是 $\mathrm{CF}_{3}$-DAST. Shibata 小组 ${ }^{[134]}$ 通过相同的 方法对该中间体进行了合成, 并发现该中间体可以与环 状酩酸酯类底物或者只含有一个活泼氢的开链酩酸酯 底物发生反应，通过 $\mathrm{C}-\mathrm{C}$ 键的断裂来实现一类新颖的 重排三氟甲硫基化反应. 随后他们发现当底物中含有两 个活泼氢时, 则会首先通过烯醇异构化, 进攻 $\mathrm{CF}_{3}$-DAST, 并且通过后续的重排反应, 来实现活泼 $\mathrm{C}$ $\mathrm{H}$ 键的三氟甲硫基化反应 ${ }^{[135]}$ (Scheme 83). 值得一提的 是，对于该两类反应，都可以通过合成不同类型的 DAST 类似物，从而实现不同类型的硫氟烷基化反应.

\section{3 自由基类型三氟甲硫基化反应}

自由基类型的亲电三氟甲硫基化反应主要有以下 两种反应模式: (1) 体系中先生成三氟甲硫基自由基再 与底物反应; (2) 体系中先生成自由基再与亲电三氟甲 硫基试剂反应(Scheme 84). 


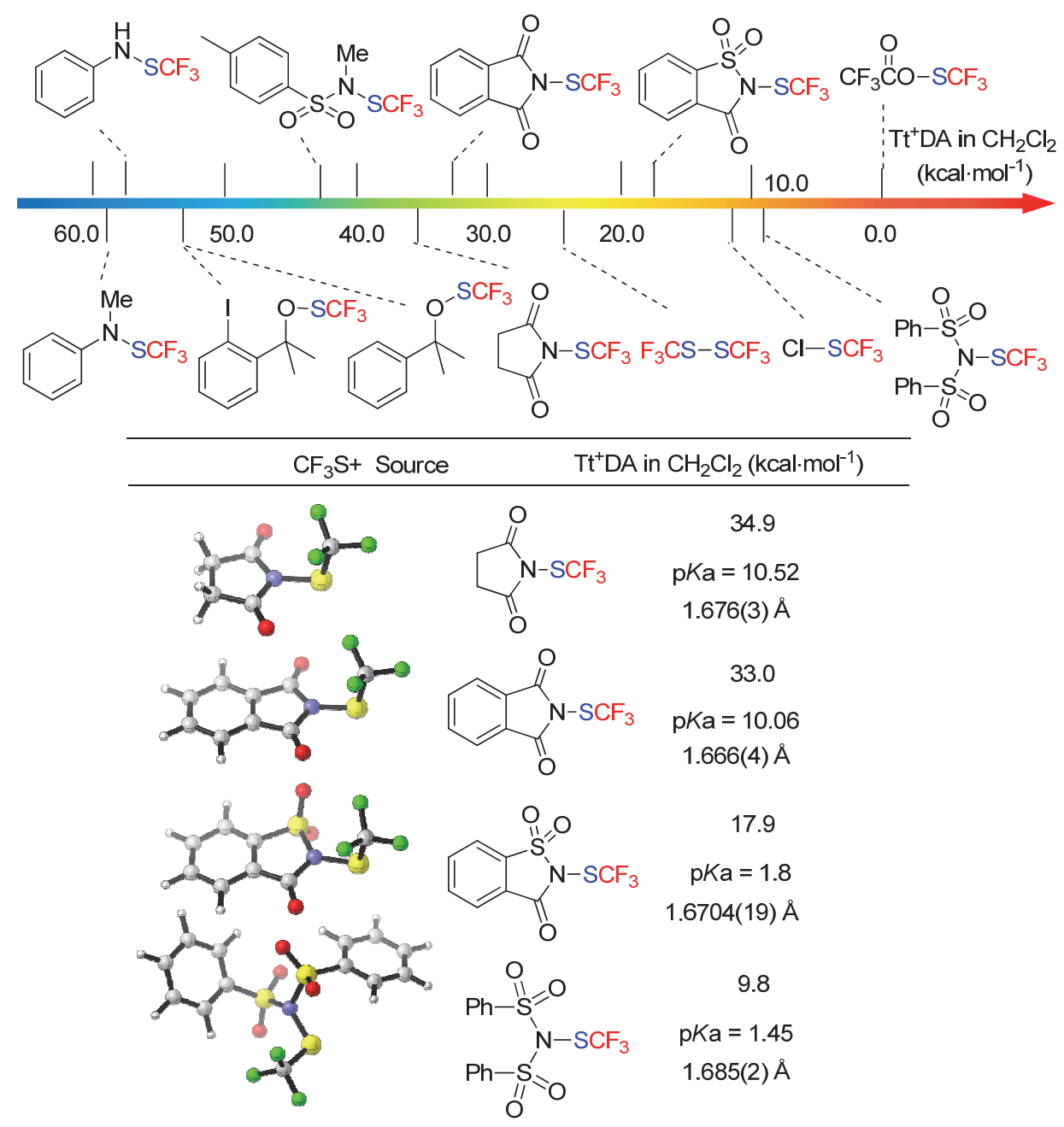

图式 82 亲电三氟甲硫基化反应活性及酰胺类型亲电三氟甲硫基化试剂的活性比较

Scheme 82 Reactivity of electrophilic trifluoromethylthiolating reagents and comparison of amide-type electrophilic trifluoromethylthiolating reagents

最早的自由基类型三氟甲硫基化反应是使用 $\mathrm{CF}_{3} \mathrm{SH}^{[136]} 、 \mathrm{CF}_{3} \mathrm{SCl}^{[137]} 、 \mathrm{CF}_{3} \mathrm{SSCF}_{3}{ }^{[138]}$ 等在紫外或者 $\mathrm{X}$ 射线的条件下产生三氟甲硫基自由基, 进而与 $\mathrm{C}-\mathrm{H}$ 键、双键等发生反应. 但是该类反应的条件较为苛刻, 且底物的选择性差，因而限制了其发展.

\subsection{1 三氟甲硫基自由基的反应}

在 2014 年, 中国科技大学的王细胜小组 ${ }^{[139]}$ 发展了 $\mathrm{AgSCF}_{3} / \mathrm{K}_{2} \mathrm{~S}_{2} \mathrm{O}_{8}$ 体系, 实现了活化烯烃的串联关环反应 (Scheme 85). 作者通过自由基关环、开环以及动力学同 位素实验支持了自由基的反应历程. 作者认为在该过程 中，三氟甲硫基自由基的产生可能有以下两种途径：一 是 $\mathrm{AgSCF}_{3}$ 被氧化成 $\mathrm{Ag}(\mathrm{II}) \mathrm{SCF}_{3}$ 后, 产生三氟甲硫基自 由基; 二是体系中的 $\mathrm{CF}_{3} \mathrm{SSCF}_{3}$ 可能会与银盐作用产生 三氟甲硫基自由基.

卿风领小组 ${ }^{[140]}$ 发现, 在 $\mathrm{AgSCF}_{3} / \mathrm{K}_{2} \mathrm{~S}_{2} \mathrm{O}_{8}$ 体系下, 加 入当量铜盐可以实现末端烯烃的三氟甲硫基化反应，从 而构建烯丙基三氟甲硫基化产物. 而由于产生的中间体
在消除时选择性较差, 产物的 $E / Z$ 选择性不高, 一般在 $6: 1$ 左右. 采用相似的策略, 作者在铜参与下也实现了 苯醌衍生物 $\mathrm{C}-\mathrm{H}$ 键的三氟甲硫基化反应 ${ }^{[141]}$ (Scheme 86).

瑞士苏黎世大学的 Nevado 小组 ${ }^{[142]}$ 采用预先构建的 底物, 通过活化的双键来捕获三氟甲硫基自由基发生自 由基串联关环反应后脱除二氧化硫，成功地构建了四环 产物(Scheme 87).

兰州大学梁永民小组 ${ }^{[143]}$ 使用 1,6-烯炔底物, 在该 体系下通过串联环化反应来构建并环骨架. 随后，他们 发现，环化反应的自由基中间体可以被过氧自由基所捕 获, 并随后脱除叔丁醇生成氧化去芳构产物 ${ }^{[144]}$ (Scheme 88).

中山大学王洪根小组 ${ }^{[145]}$ 采用炔酸苯酯类衍生物, 在该体系下通过自由基串联环化反应成功构建了三氟 甲硫基取代的香豆素类衍生物(Scheme 89). 而采用硫 氯酸银也可以实现硫氧基取代的香豆素衍生物的合成. 

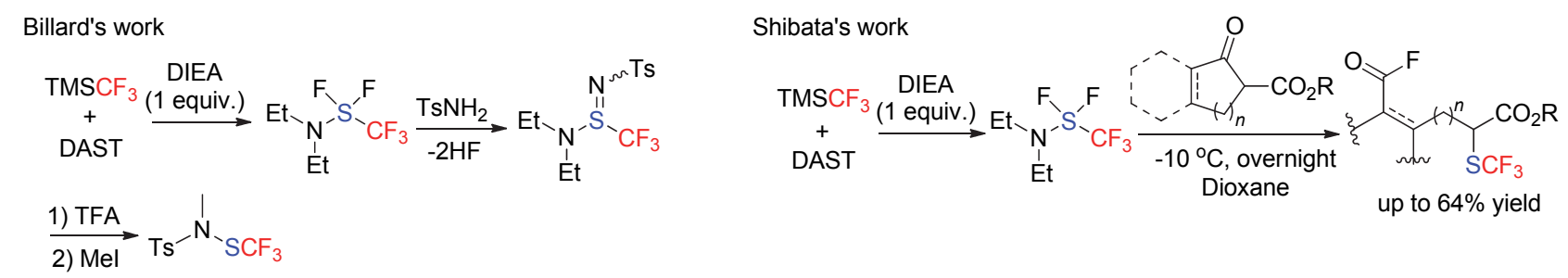

$\mathrm{C}-\mathrm{C}$ bond cleavage
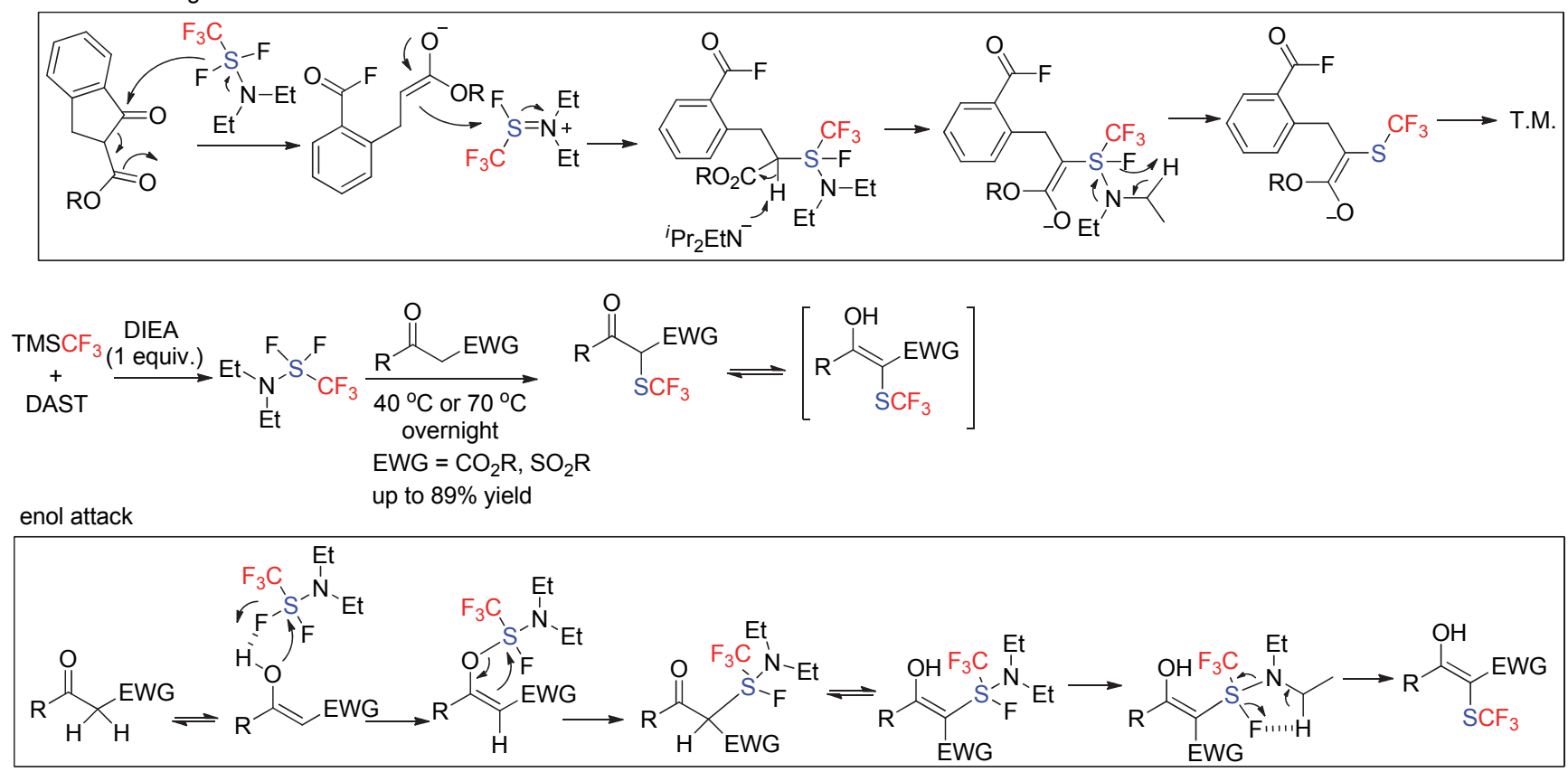

图式 $83 \quad \mathrm{CF}_{3}$-DAST 作为三氟甲硫基源的重排反应

Scheme $83 \quad \mathrm{CF}_{3}$-DAST as trifluoromethylthiol source

$$
\left[\cdot \mathrm{SCF}_{3}\right]+\mathrm{R}-\mathrm{X} \longrightarrow \mathrm{R}-\mathrm{SCF}_{3} \longleftarrow \mathrm{R} \cdot+\left[\mathrm{SCF}_{3}{ }^{+}\right]
$$

图式 84 两种自由基类型的三氟甲硫基反应

Scheme 84 Two types of radical trifluoromethylthiolating reactions

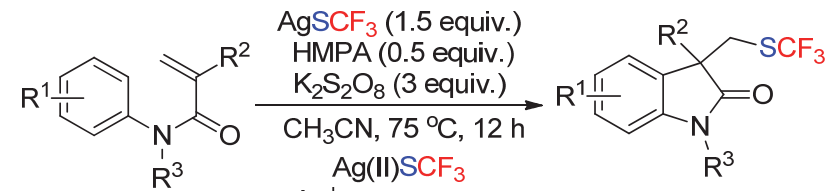

$\mathrm{Ag}^{+}+\mathrm{F}_{3} \mathrm{CSSCF}_{3} \quad$ up to $91 \%$ yield $\mathrm{F}_{3} \mathrm{CS} \cdot$ Radical-type path

图式 85 活化烯烃 $\mathrm{AgSCF}_{3} / \mathrm{K}_{2} \mathrm{~S}_{2} \mathrm{O}_{8}$ 体系下自由基三氟甲硫基化反应 Scheme 85 Activated alkenes radical trifluoromethylthiolating reaction using $\mathrm{AgSCF}_{3} / \mathrm{K}_{2} \mathrm{~S}_{2} \mathrm{O}_{8}$

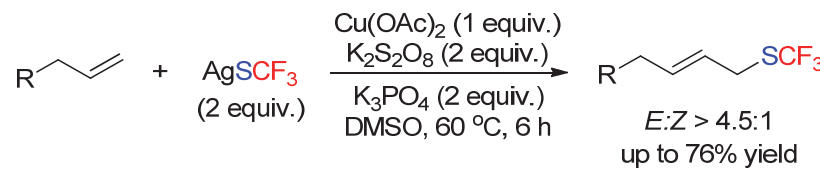

$$
\begin{aligned}
& \text { 作 }
\end{aligned}
$$

图式 $86 \mathrm{AgSCF}_{3} / \mathrm{K}_{2} \mathrm{~S}_{2} \mathrm{O}_{8}$ 在铜盐氧化下的自由基三氟甲硫基化反应 Scheme 86 Copper-mediated oxidative trifluoromethylthiolation of alkenes and quinones

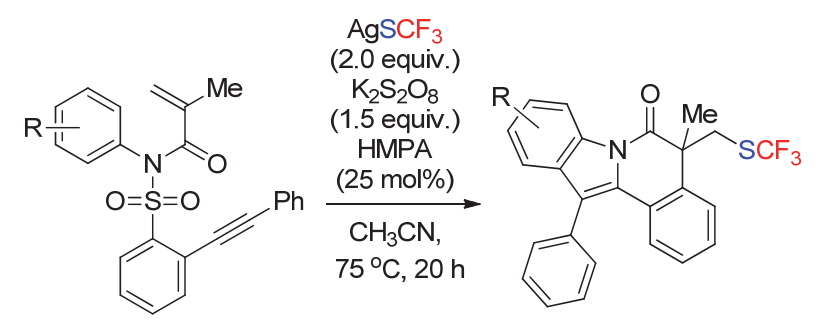

图式 $87 \mathrm{AgSCF}_{3} / \mathrm{K}_{2} \mathrm{~S}_{2} \mathrm{O}_{8}$ 体系下串联环化反应

Scheme 87 Cyclization cascades under $\mathrm{AgSCF}_{3} / \mathrm{K}_{2} \mathrm{~S}_{2} \mathrm{O}_{8}$ system

上海有机所卿凤翎小组 ${ }^{[146]}$ 从肉桂酸出发, 在外加 铜盐和氧化剂的条件下实现了氧化脱酸三氟甲硫基化 反应. 对于该反应, 可以得到反式为主的产物, 顺反选 择性最高可达 $11 ： 1$. 而当采用铁盐为氧化剂, 使用乙 腈和水为混合溶剂时，可以进一步对自由基氧化后所形 成碳正离子进行水和, 并在氧气的氛围下进一步氧化成 羰基结构(Scheme 90).

华东理工大学的曹松小组 ${ }^{[147]}$ 从炔烃出发, 通过自 由基类型的三氟甲硫化反应，可以实现马氏与反马氏选 择性的氢-三氟甲硫基化反应(Scheme 91). 对于马氏选 择性反应, 底物中需要连有烷氧基等给电子基才. 而对 于马氏选择性反应，存在着顺反选择性问题，其比例接 近于 $1: 1$. 但是该体系对于非苯乙烯类的烯烃底物, 反 
应并不适用.
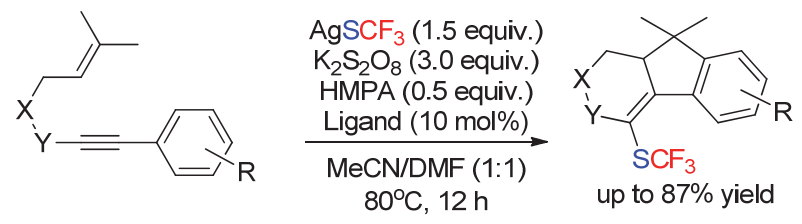

$\mathrm{X}=\mathrm{NTs}, \mathrm{C}\left(\mathrm{CO}_{2} \mathrm{Me}\right)_{2}, \mathrm{Y}=\mathrm{CH}_{2}$ $\mathrm{X}=\mathrm{O}, \mathrm{Y}=$ carbonyl group
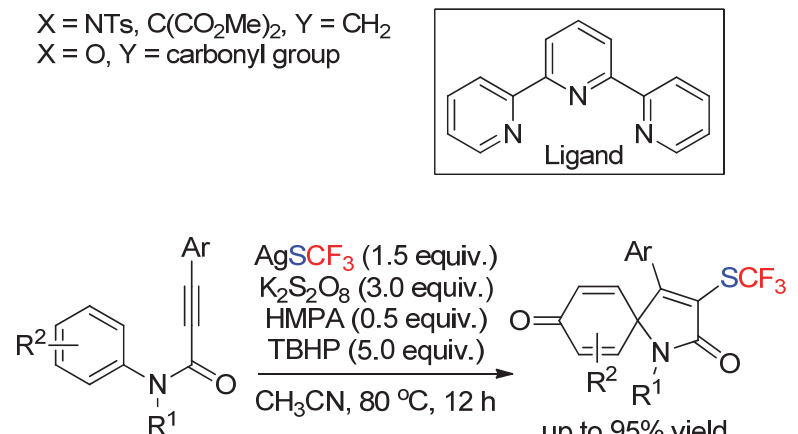
$\mathrm{R}^{1}$<smiles>[R]C1=CC(OCC)C=CC12C([Al])=C([Si](F)(F)F)C(=O)N2[R]</smiles>

图式 881,6 -烯炔底物在 $\mathrm{AgSCF}_{3} / \mathrm{K}_{2} \mathrm{~S}_{2} \mathrm{O}_{8}$ 体系下串联环化反应

Scheme 88 Radical cascade cyclization of 1,6-enynes under $\mathrm{AgSCF}_{3} /$ $\mathrm{K}_{2} \mathrm{~S}_{2} \mathrm{O}_{8}$ system<smiles>[R]C#CC(=O)Oc1cc#[R]cc1</smiles>
$\mathrm{R}^{2}$

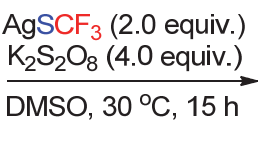

DMSO, $30^{\circ} \mathrm{C}, 15 \mathrm{~h}$

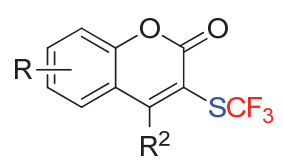

up to $80 \%$ yield
图式 89 炔酸苯酯类衍生物在 $\mathrm{AgSCF}_{3} / \mathrm{K}_{2} \mathrm{~S}_{2} \mathrm{O}_{8}$ 体系下串联环化反应 Scheme 89 Radical trifluoromethylthiolation of aryl alkynoate esters under $\mathrm{AgSCF}_{3} / \mathrm{K}_{2} \mathrm{~S}_{2} \mathrm{O}_{8}$ system

$$
\text { (2.0 equiv.) }
$$

图式 $90 \mathrm{AgSCF}_{3} / \mathrm{K}_{2} \mathrm{~S}_{2} \mathrm{O}_{8}$ 体系下肉桂酸衍生物脱羧三氟甲硫基化反应 Scheme 90 Decarboxylative trifluoromethylthiolation of cinnamic acid under $\mathrm{AgSCF}_{3} / \mathrm{K}_{2} \mathrm{~S}_{2} \mathrm{O}_{8}$ system

上海有机所施敏小组 ${ }^{[148]}$ 使用亚甲基环丙烷底物, 在该体系下通过三元环开环和随后的自由基成环反应, 构建了烯基三氟甲硫基化产物. 该产物在过硫酸钠作为 氧化剂的条件下, 可以进一步生成芳构化的三氟甲硫基 化产物(Scheme 92).

美国西弗吉尼亚大学的 Hoover 小组 ${ }^{[149]}$ 发现, 对于 香豆素类羧酸衍生物, 可以在该体系下以优秀的收率实
现氧化脱羧三氟甲硫基化反应(Scheme 93).

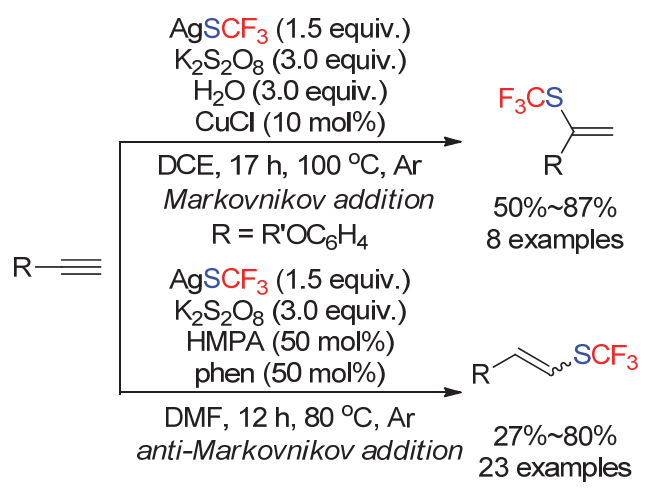

图式 $91 \mathrm{AgSCF}_{3} / \mathrm{K}_{2} \mathrm{~S}_{2} \mathrm{O}_{8}$ 体系下炔烃马氏、反马氏氢/三氟甲硫基化 Scheme 91 Anti-Markovnikov and Markovnikov-selective hydrotrifluoromethylthiolation of terminal alkyne under $\mathrm{AgSCF}_{3} / \mathrm{K}_{2} \mathrm{~S}_{2} \mathrm{O}_{8}$ system

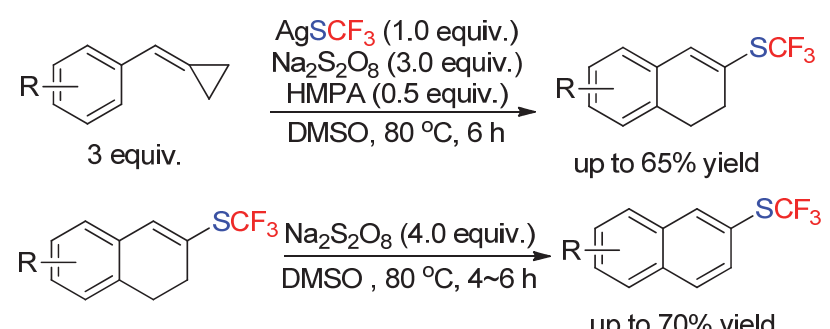

up to $70 \%$ yield

图式 $92 \mathrm{AgSCF}_{3} / \mathrm{K}_{2} \mathrm{~S}_{2} \mathrm{O}_{8}$ 体系下亚甲基环丙烷底物的开环串联反应 Scheme 92 Trifluoromethylthiolation of methylenecyclopropane under $\mathrm{AgSCF}_{3} / \mathrm{K}_{2} \mathrm{~S}_{2} \mathrm{O}_{8}$ system

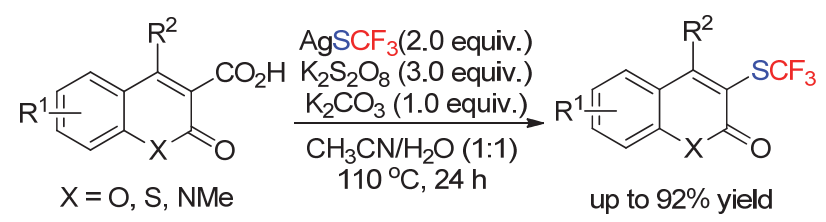

图式 $93 \mathrm{AgSCF}_{3} / \mathrm{K}_{2} \mathrm{~S}_{2} \mathrm{O}_{8}$ 体系下香豆素类羧酸脱羧三氟甲硫基化反应 Scheme 93 Decarboxylative trifluoromethylthiolation of coumarin-3-carboxylic acid under $\mathrm{AgSCF}_{3} / \mathrm{K}_{2} \mathrm{~S}_{2} \mathrm{O}_{8}$ system

华东理工大学的刘培念小组 ${ }^{[150]}$ 报道了烯丙醇底物 在自由基条件下的半频哪醇重排反应(Scheme 94). 在 该反应中, 吡啶的加入对产率的提高至关重要, 其在反 应体系中起着缚酸剂的作用.

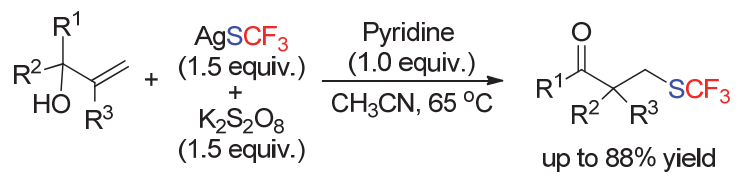

图式 $94 \mathrm{AgSCF}_{3} / \mathrm{K}_{2} \mathrm{~S}_{2} \mathrm{O}_{8}$ 体系下半频哪醇重排三氟甲硫基化反应 Scheme 94 Trifluoromethylthiolation of diaryl allylic alcohols via radical neophyl rearrangement under $\mathrm{AgSCF}_{3} / \mathrm{K}_{2} \mathrm{~S}_{2} \mathrm{O}_{8}$ system

Glorius 小组采用光催化的策略，使用邻苯二甲酰 亚胺试剂, 同样可以产生三氟甲硫基自由基. 使用该策 略, 作者实现了烯烃 ${ }^{[151]}$, 烯丙醇三氟磺酸酯 ${ }^{[151]}$ 以及丙 烯酰胺 ${ }^{[151]}$ 等底物的三氟甲硫基化反应(Scheme 95). 作 
者在反应体系中加入了当量的四丁基卤化铵, 其作用可 能是生成活性更高的 $\mathrm{CF}_{3} \mathrm{~S}-\mathrm{X}$ 中间体.

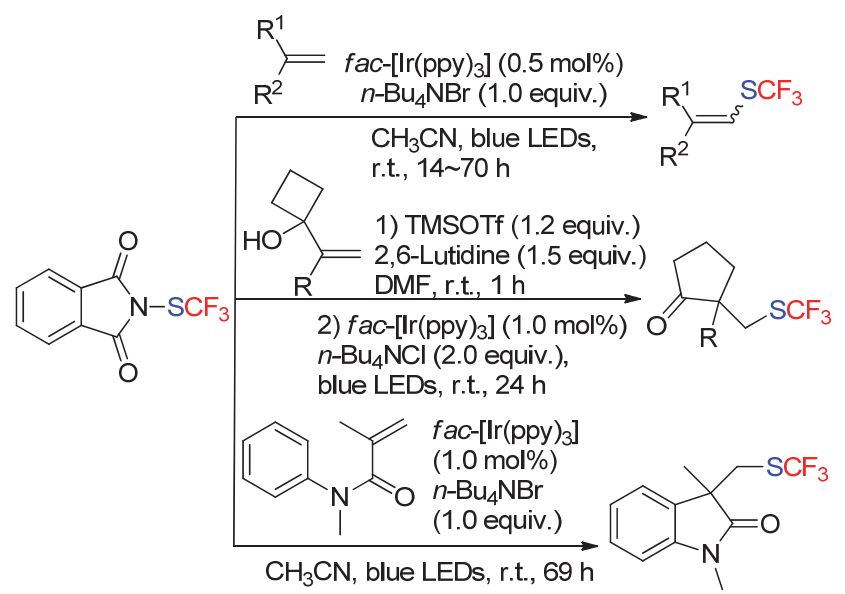

图式 $95 \mathrm{~N}$-三氟甲硫基邻苯二甲酰亚胺在光催化条件下的自由基三 氟甲硫基化反应

Scheme 95 Radical trifluoromethylthiolation under photocatalytic conditions using $N$-(trifluoromethylthio)phthalimide as $\mathrm{SCF}_{3}$ source

东京工业大学 Akita 小组 ${ }^{[152]}$ 在光催化的条件下, 采 用 $N$-三氟甲硫基丁二酰亚胺试剂实现了双官能团化反 应(Scheme 96). 该反应首先经过单电子还原产生三氟 甲硫基自由基，随后进攻苯乙烯衍生物，生成的茮基自 由基经过单电子氧化后接受水的进攻从而实现双官能 团化反应. 此外, 当使用二氯甲烷和醇为混合溶剂时, 可以实现烷氧基化三氟甲硫基双官能团化反应.

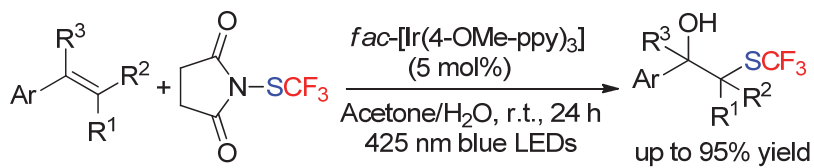

图式 $96 \mathrm{~N}$-三氟甲硫基丁二酰亚胺在光催化条件下的自由基三氟甲 硫基化反应

Scheme 96 Radical trifluoromethylthiolation under photocatalytic conditions using $N$-(trifluoromethylthio)succinimide as $\mathrm{SCF}_{3}$ source

法国的 Magnier 小组与 Billard 小组 ${ }^{[153]}$ 合作, 发现 使用糖精试剂在光催化条件下可以产生三氟甲硫基自 由基，并且通过电子顺磁共振检测到了三氟甲硫基自由 基的存在(Scheme 97). 采用该体系, 作者实现了分子内 的串联环化反应以及苯乙烯衍生物的双官能团化反应.

\subsection{2 自由基与亲电三氟甲硫基试剂的反应}

自由基类型的三氟甲硫基化反应的另外一种反应 是体系中首先产生一个活性的自由基中间体, 随后亲电 型的三氟甲硫基试剂来作为自由基淬灭剂, 实现三氟甲 硫基化反应。

上海有机所的吕龙和沈其龙小组 ${ }^{[154]}$ 首先报道了该 类反应. 他们采用烷基羧酸以及烷基硼酸钾盐, 在水 相、银催化条件下实现了脱羧三氟甲硫基化反应. 在该 反应中, SDS 的加入至关重要, 作者推测该反应可能是 在胶束中进行的, 生成的烷基自由基随后被亲电三氟甲
硫基试剂淬灭，生成三氟甲硫基产物. 在此工作的基础 上，作者随后采用 $\mathrm{Fe}$ 盐和硼烷产生 $[\mathrm{Fe}-\mathrm{H}]$ 物种后，对烯 烃进行加成，生成烷基自由基后与次磺酸酯试剂反应, 实现了未活化烯烃马氏选择性的氢化三氟甲硫基化反 应 $^{[155]}$ (Scheme 98).

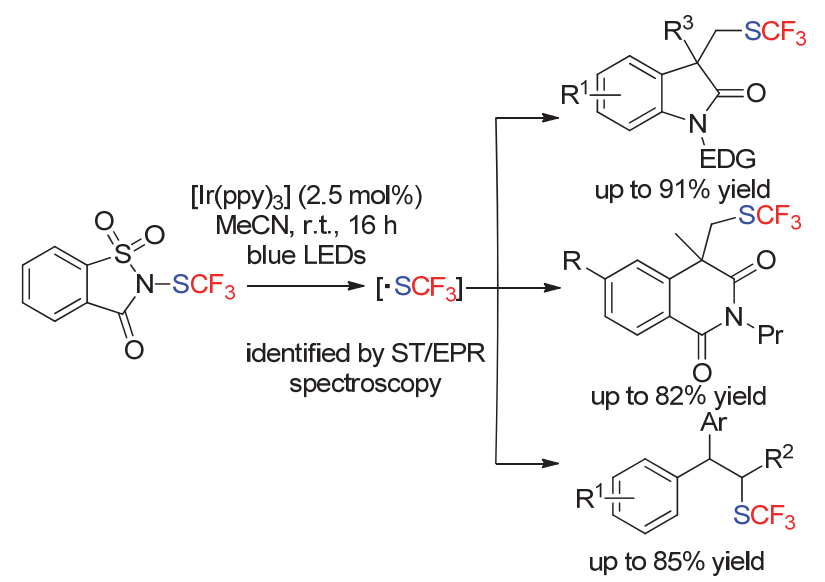

图式 97 Shen 试剂在光催化条件下的自由基三氟甲硫基化反应 Scheme 97 Radical trifluoromethylthiolation under photocatalytic conditions using $N$-(trifluoromethylthio)saccharin as $\mathrm{SCF}_{3}$ source

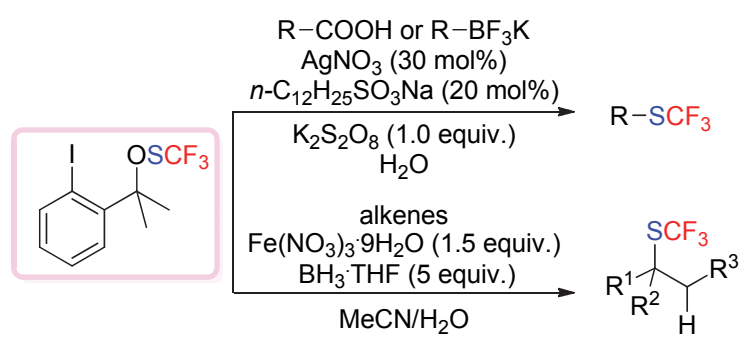

图式 $98 \mathrm{Lu}$ and Shen 试剂作为自由基淬灭剂的反应 Scheme $98 \mathrm{Lu}$ and Shen reagent as radical quencher

Glorius 小组 ${ }^{[156]}$ 利用光催化的策略，在金属铱或者 有机染料作为光敏剂的条件下, 实现烷基羧酸的脱羧三 氟甲硫基化反应(Scheme 99). 在此条件下，烷基自由基 的形成通过光氧化过程实现，因而不用外加的氧化剂便 可以产生烷基自由基，从而与亲电三氟甲硫基试剂进行 反应. 对于一级羧酸底物, 也可以很好地发生转化.

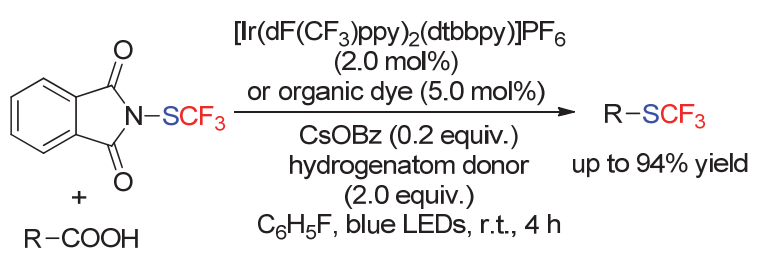

图式 99 光催化条件下的脱羧三氟甲硫基化反应

Scheme 99 Visible light-promoted decarboxylative trifluoromethylthiolation of alkyl carboxylic acids

上海有机所的陈庆云小组 ${ }^{[157]}$ 发现，使用选择性氟 化试剂(selectfluor)也可以作为体系中的氧化剂，从而实 现脱羧三氟甲硫基化反应(Scheme 100). 该反应的机理 
可能是 $\mathrm{AgSCF}_{3}$ 被选择性氟化试剂氧化生成 $\mathrm{Ag}(\mathrm{II}) \mathrm{SCF}_{3}$. 该物种作为氧化剂对羧酸进行氧化脱羧并淬灭, 形成最 终的脱羧三氟甲硫基产物.

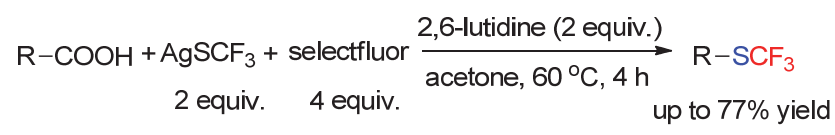

图式 100 氧化条件下的脱羧三氟甲硫基化反应

Scheme 100 Oxidative decarboxylative trifluoromethylthiolation of alkyl carboxylic acids

上海有机所的陈庆云小组 ${ }^{[158 a]}$ 与南开大学的汤平平 小组 ${ }^{[158 b]}$ 几乎同时报道了首例烷基 $\mathrm{C}-\mathrm{H}$ 键自由基类型 的三氟甲硫基化反应(Scheme 101). 该类反应的机理可 能是体系中产生的硫酸根自由基首先篗取烷基 $\mathrm{C}-\mathrm{H}$ 形 成烷基自由基，随后与体系中的 $\mathrm{Ag}(\mathrm{II}) \mathrm{SCF}_{3} 、 \mathrm{CF}_{3} \mathrm{SSCF}_{3}$ 或者是 $\left[\mathrm{SCF}_{3} \bullet\right.$ 自由基发生反应从而形成最终的 $\mathrm{C}-\mathrm{H}$ 键 三氟甲硫基化产物.

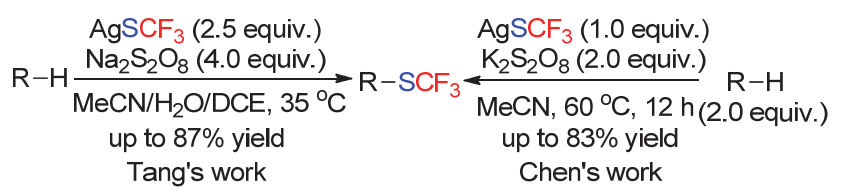

图式 101 非活化烷基 $\mathrm{C}-\mathrm{H}$ 键自由基三氟甲硫基化反应

Scheme 101 Unactivated $\mathrm{C}-\mathrm{H}$ bond trifluoromethylthiolation under radical conditions

Glorius 小组 ${ }^{[159]}$ 采用光催化的策略, 使用苯甲酸钠 为碱, 实现了烷基 $\mathrm{C}-\mathrm{H}$ 的三氟甲硫基化反应(Scheme 102). 相比较于陈庆云小组和汤平平小组的工作, 该工 作的反应条件更加温和, 并且有着更优秀的三级 $\mathrm{C}-\mathrm{H}$ 键的区域选择性.

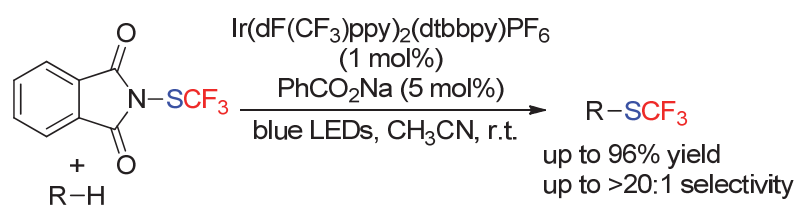

图式 102 光催化条件下非活化烷基 $\mathrm{C}-\mathrm{H}$ 键三氟甲硫基化反应

Scheme 102 Radical trifluoromethylthiolation of unactivated $\mathrm{C}-\mathrm{H}$ bond under photocatalytic conditions

最近, 山东大学的徐政虎小组 ${ }^{[160]}$ 采用金催化/光催 化协同催化的策略, 采用三氟甲硫基苯基砜结构的试 剂, 实现了苯乙烯衍生物的双官能团化反应(Scheme 103). 该反应的机理可能是在光催化条件下, 活性的阳 离子一价金物种 $\operatorname{IPrAu}(\mathrm{I})$ 首先被还原成零价金 $\operatorname{IPrAu}(0)$. 该零价金被三氟甲硫基苯基砜还原, 生成 $\mathrm{IPrAuSCF}_{3}$ 与 砜基自由基. 砜基自由基对苯乙烯底物进行自由基加 成, 生成的烷基自由基与亲电型的 $\mathrm{IPrAuSCF}_{3}$ 结合生成 二价金中间体，随后被三价 $\mathrm{Ru}$ 催化剂氧化生成三价金 中间体. 该三价金中间体发生还原消除生成最终产物, 同时生成一价金物种继续催化循环. 该反应有着高的原 子经济性. 除了三氟甲硫基以外, 硫烷基以及硫苯基类
型的试剂也可以很好地实现相应的双官能团化反应.
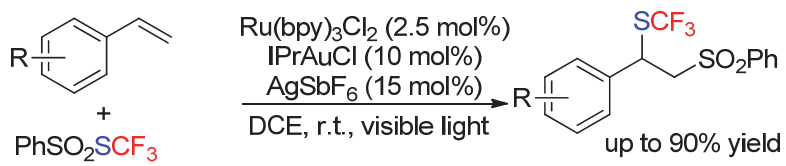

$[\operatorname{IPrAu}(\mathrm{I})]^{+}[\mathrm{Ru}(\mathrm{bpy})]^{3+} \mathrm{PhSO}_{2} \mathrm{SCF}_{3} \operatorname{IPrAu}(\mathrm{I}) \mathrm{SCF}_{3}$

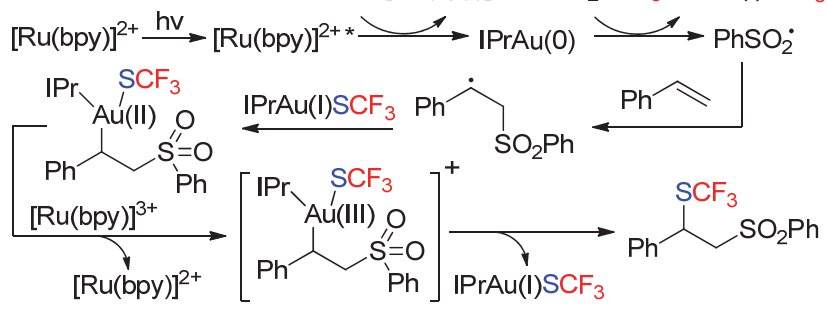

图式 103 金催化/光催化协同催化下烯烃的砜基三氟甲硫基化反应 Scheme 103 Dual gold and photoredox catalysis: visible light-mediated intermolecular atom transfer trifluoromethylthiosulfonylation of alkene

\section{3 总结与展望}

从以上总结中, 我们可以看到，在近十年来的时间 里，三氟甲硫基化反应得到了快速的发展，其中包括过 渡金属催化的反应、自由基类型的反应以及不同类型的 亲电三氟甲硫基试剂的开发. 这些都为化学工作者以及 药物化学工作者方便简洁地引入三氟甲硫基团提供了 一个较为全面的工具箱.

但与此同时，该领域仍然存在着一些问题与挑战需 要我们去解决. 首先, 对于直接三氟甲硫基化反应而言, 通常都需要用到三氟甲硫基金属化合物如 $\mathrm{AgSCF}_{3}$ 和 $\mathrm{CuSCF}_{3}$, 这在成本是比较昂贵的. 其次, 不对称三氟甲 硫基化研究还处于起步阶段, 其相关报道基本都集中于 酮酸酯和吲哚酮底物, 对于其他底物如羰基 $\alpha$ 位的不对 称三氟甲硫基化反应或许会成为一个重要的研究课题.

最后, 我们也认为, 对于目前已经存在的含三氟甲 硫基的药物、农药分子, 对其相关的代谢途径的研究可 能会为我们更好地设计和合成含三氟甲硫基的化合物 提供更进一步的理论支持.

\section{作者简介}

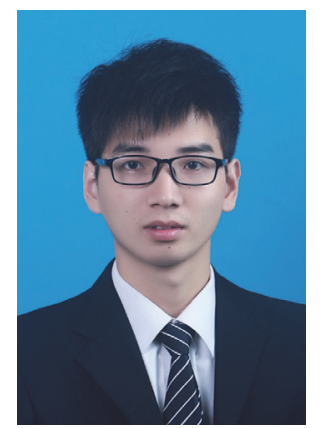

张盼盼, 男, 中国科学院上海有机化学研究所在读博士 生, 主要从事亲电三氟甲硫基化试剂的开发以及小分子三氟 甲硫基化反应的研究. 


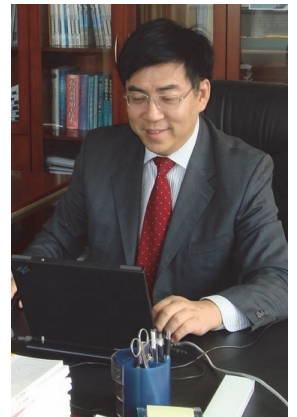

吕龙, 男, 1991 年获中国科学院上海有机化学研究所博 士学位, 1993 年至 1996 年在世界氟化学研究中心美国杜邦公 司研究与发展中心和衣阿华大学从事博士后研究, 1996 年 10 月至今于中国科学院上海有机化学研究所开展独立研究工作. 中国科学院“百人计划”入选者（1997 年），国家杰出青年基金 获得者（1998 年），国家级有突出贡献的中青年专家 (1998 年). 现主要从事新型含氟砌块的设计、合成及反应研究、含 氟功能材料以及新农药的创制等研究工作.

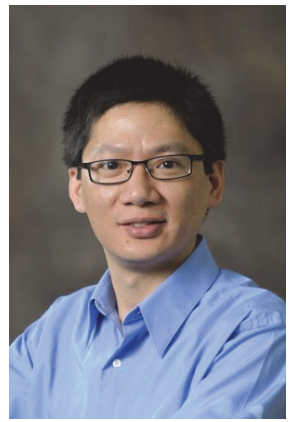

沈其龙, 男, 1996 年 7 月本科毕业于南京大学环境科学 与工程系; 1999 年 7 月硕士毕业于上海有机化学研究所; 2002 年7月毕业于美国麻省大学 Dartmouth 分校, 获理学硕士学位; 2002 年 9 月至 2007 年 9 月就读于美国耶鲁大学, 获哲学博士 学位. 2007 年 10 月至 2010 年 3 月于美国伊利诺伊斯大学香槟 分校化学系从事博士后研究; 2010 年 4 月至今, 于中国科学院 上海有机化学研究所开展独立研究工作. 中国科学院 “百人计 划” 入选者, 国家杰出青年基金获得者（2017 年）。现主要从 事亲电氟烷基化试剂的创制及反应研究; 金属有机氟化学, 氟烷基取代的过渡金属络合物的合成、结构表征及反应; 具有 应用前景的含氟物质及含氟功能材料的合成等研究工作.

\section{References}

[1] 《2016 研究前沿》

[2] (a) Leo, A.; Hansch, C.; Elkins, D. Chem. Rev. 1971, 71, 525. (b) Hansch, C.; Leo, A.; Taft, R. W. Chem. Rev. 1991, 91, 165.

[3] (a) Boiko, V. N. Beilstein J. Org. Chem. 2010, 6, 880. (b) Liang, T.; Neumann, C. N.; Ritter, T. Angew. Chem. Int. Ed. 2013, 52, 8214. (c) He, W.-M.; Weng, Z.-Q. Prog. Chem. 2013, 25, 1071 (in Chinese). (何伟明, 翁志强, 化学进展, 2013, 25, 1071). (d) Fang, L. Huaxue Tongbao, 2014, 77, 1058 (in Chinese). (方玲, 化学通报, 2014, 77, 1058). (e) Liu, Z.-J.; Liu, P.; Wu, F.-H. J. Shanghai Instit. of Tech.(Nat. Sci.). 2014, 14, 93. (f) Toulgoat, F.; Alazet, S.; Billard, T. Eur. J. Org. Chem. 2014, 2415. (g) Chu, L.-L.; Qing, F.-L. Acc. Chem. Res. 2014, 47, 1513. (h) Shao, X.-X.; Xu, C.-F.; Lu, L.; Shen, Q.-L. Acc. Chem. Res. 2015, 48, 1227. (i) Xu, X. H.; Matsuzaki, K.; Shibata, N. Chem. Rev. 2015, 115, 731. (j) Zhang, K.; Xu, X.-H.; Qing, F.-L. Chin. J. Org. Chem. 2015, 35, 556 (in Chinese). (张柯, 徐修华, 卿风领, 有机化学, 2015, 35, 556). (k) Yang, X.-Y.; Wu, Tao.; Phipps, R. J.; Toste, F. D. Chem. Rev. 2015, 115, 826. (1) Lin, J.-H.; Ji, Y.-L.; Xiao, J.-C. Current Org. Chem. 2015, 19, 1541. (m) Chachignon, H.; Cahard, D. Chin. J. Chem. 2016, 34, 445. (n)
Zheng, H.-D.; Huang, Y.-J.; Weng, Z.-Q. Tetrahedron Lett. 2016, 57 , 1397. (o) Guo, Y.; Huang, M.-W.; Fu, X.-L.; Liu, C.; Chen, Q.-Y.; Zhao, Z.-G.; Zeng, B.-Z.; Chen, J. Chin. Chem. Lett. 2017, 28, 719. (p) Li, G.-M.; Sun, D.-Q. Chin. J. Org. Chem. 2016, 36, 1715 (in Chinese). (李恭铭, 孙德群, 有机化学, 2016, 36, 1715).

[4] (a) Fluorverbindungen, U. Angew. Chem. 1939, 52, 457. (b) Yagupolski, L. M.; Marenets, M. S. J. Gen. Chem. U.S.S.R. 1954, 24, 885.

[5] (a) Ruppert, I.; Schlich, K.; Volbach, W. Tetrahedron Lett. 1984, 25 , 2195. (b) Kolomeitsev, A. A.; Movchun, V. N.; Kondranenko, N. V.; Yagupolski, Y. L. Synthesis 1990, 1151. (c) Shono, T.; IShifune, M.; Okada, T.; Kashimura, S. J. Org. Chem. 1991, 56, 2. (d) Billard, T.; Large, S.; Langlois, B. R. Tetrahedron Lett. 1997, 38, 65. (e) Singh, R. P.; Cao, G.; Kirchmeier, R. L.; Shreeve, J. J. Org. Chem. 1999, 64, 2873. (f) Folleas, B.; Marek, I.; Normant, J. F.; Saint-Jalmes, L. Tetrahedron 2000, 56, 275. (g) Large, S.; Roques, N.; Langlois, B. R. J. Org. Chem. 2000, 65, 8848. (h) Billard, T.; Langlois, B. R.; Blond, G. Eur. J. Org. Chem. 2001, 1467. (i) Steensma, R. W.; Galabi, S.; Tagat, J. R.; McCombie, S. W. Tetrahedron Lett. 2001, 42, 2281. (j) Potash, S.; Rozen, S. J. Fluorine Chem. 2014, 168, 173.

[6] (a) Yagupolski, L. M.; Kondranenko, N. V.; Timofeeva, G. N. Zh. Org. Khim. 1984, 20, 115. (b) Umemoto, T. Chem. Rev. 1996, 96, 1757. (c) Yang, J. J.; Kirchmeier, R. L.; Shreeve, J. J. Org. Chem. 1998, 63, 2656. (d) Eisenberger, P.; Gischig, S.; Togni, A. Chem Eur. J. 2006, 12, 2579. (e) Kieltsch, I.; Eisenberger, P.; Togni, A. Angew. Chem. Int. Ed. 2007, 46, 754. (f) Koller, R.; Stanek, K.; Stolz, D.; Ardoom, R.; Niedermann, K.; Togni, A. Angew. Chem. Int. Ed. 2009, 48, 4332 .

[7] (a) Boiko, V. N.; Shchupak, G. M.; Yagupolski, L. M. Zh. Org. Khim. 1977, 13, 1057. (b) Popov, I.; Boiko, V. N.; Kondranenko, N. V.; Sambur, V. P.; Yagupolski, L. M. 1977, 13, 2135. (c) Boiko, V. N.; Dashevskaya, T. A.; Shchupak, G. M.; Yagupolski, L. M. Zh. Org. Khim. 1979, 15, 396.

[8] Man, E. H.; Coffman, D. D.; Muetterties, E. L. J. Am. Chem. Soc. $\mathbf{1 9 5 9}, 81,3575$

[9] Emeleus, H. J.; MacDuffie, D. E. J. Am. Chem. Soc. (Resumed) 1961, 83, 2572.

[10] Yagupolskii, L. M.; Kondratenko, N. V.; Sambur, V. P. Synthesis 1975, 1975, 721 .

[11] Tyrra, W.; Naumann, D.; Hoge, B.; Yagupolskii, Y. L. J. Fluorine Chem. 2003, 119, 101.

[12] Remy, D. C.; Rittle, K. E.; Hunt, C. A.; Freedman, M. B. J. Org. Chem. 1976, 41, 1644.

[13] Kondratenko, N. V.; Kolomeytsev, A. A.; Popov, V. I.; Yagupolskii, L. M. Synthesis 1985, 667.

[14] Chen, Q.-Y.; Duan, J.-X. J. Chem. Soc., Chem. Commun. 1993, 918.

[15] Clark, J. H.; Tavener, S. J. J. Fluorine Chem. 1997, 85, 169.

[16] Teverovskiy, G.; Surry, D. S.; Buchwald, S. L. Angew. Chem. Int. Ed. 2011, 50, 7312 .

[17] Zhang, C.-P.; Vicic, D. A. J. Am. Chem. Soc. 2012, 134, 183.

[18] Yin, G.; Kalvet, I.; Englert, U.; Schoenebeck, F. J. Am. Chem. Soc. 2015, 137, 4164.

[19] Yin, G.; Kalvet, I.; Schoenebeck, F. Angew. Chem. Int. Ed. 2015, 54 6809.

[20] Dürr, A. B.; Yin, G.; Kalvet, I.; Napoly, F.; Schoenebeck, F. Chem. Sci. 2016, 7, 1076.

[21] Nguyen, T.; Chiu, W.-L.; Wang, X.-Y.; Sattler, M. O.; Love, J. A. Org. Lett. 2016, 18, 5492.

[22] Chen, C.; Xie, Y.; Chu, L.-L.; Wang, R.-W.; Zhang, X.; Qing, F. -L. Angew. Chem. Int. Ed. 2012, 51, 2492.

[23] Chen, C.; Chu, L.-L.; Qing, F.-L. J. Am. Chem. Soc. 2012, 134, 12454.

[24] Zhang, C.-P.; Vicic, D. A. Chem. Asian J. 2012, 7, 1756.

[25] Zhai, L.-J.; Li, Y.-M.; Yin, J.; Jin, K.; Zhang, R.; Fu, X.-M.; Duan, C.-Y. Tetrahedron 2013, 69, 10262.

[26] Zhao, M.-Z.; Zhao, X.-M.; Zheng, P.-R.; Tian, Y.-W. J. Fluorine Chem. 2017, 194, 73

[27] Wu, W.; Wang, B.-Y.; Ji, X.-F.; Cao, S. Org. Chem. Front. 2017, DOI: $10.1039 / \mathrm{c} 7 \mathrm{qo} 00198 \mathrm{c}$.

[28] Chen, C.; Xu, X.-H.; Yang, B.; Qing, F.-L. Org. Lett. 2014, 16, 3372.

[29] Yin, W.; Wang, Z.; Huang, Y. Adv. Synth. Catal. 2014, 356, 2998.

[30] Liu, X.-G.; Li, Q.-J.; Wang, H.-G. Adv. Synth. Catal. 2017, 359, 1942.

[31] Munavalli, S.; Rossman, D. I.; Rohrbaugh, D. K.; Ferguson, C. P.; Hsu, F. L. Heteroat. Chem. 1992, 3, 189

[32] Rheingold, A. L.; Munavalli, S.; Rossman, D. I.; Ferguson, C. P. In- 
org. Chem. 1994, 33, 1723.

[33] Weng, Z.-Q.; He, W.; Chen, C.; Lee, R.; Tan, D.; Lai, Z.; Kong, D.; Yuan, Y.; Huang, K.-W. Angew. Chem. Int. Ed. 2013, 52, 1548.

[34] Wang, Z.; Tu, Q.; Weng, Z.-Q. J. Organomet. Chem. 2014, 751, 830.

[35] Yang, Y.; Xu, L.; Yu, S.; Liu, X.; Zhang, Y.; Vicic, D. A. Chem. Eur. J. 2016, 22, 858 .

[36] Zhang, M.; Weng, Z.-Q. Adv. Synth. Catal. 2016, 358, 386.

[37] (a) Kong, D.; Jiang, Z.; Xin, S.; Bai, Z.; Yuan, Y.; Weng, Z.-Q. Tetrahedron 2013, 69, 6046. (b) Huang, Y.; He, X.; Li, H.; Weng, Z.-Q. Eur. J. Org. Chem. 2014, 2014, 7324. (c) Lin, Q.; Chen, L.; Huang, Y.; Rong, M.; Yuan, Y.; Weng, Z.-Q. Org. Biomol. Chem. 2014, 12, 5500 .

[38] (a) Zhu, P.; He, X.; Chen, X.; You, Y.; Yuan, Y.; Weng, Z.-Q. Tetrahedron 2014, 70, 672. (b) Huang, Y.; Ding, J.; Wu, C.; Zheng, H.; Weng, Z.-Q. J. Org. Chem. 2015, 80, 2912.

[39] Zhang, M.; Chen, J.; Chen, Z.; Weng, Z.-Q. Tetrahedron 2016, 72, 3525 .

[40] Kondratenko, N. V.; Sambur, V. P. Ukr. Khim. Zh. (Russ. Ed.) 1975, $41,516$.

[41] Adams, D. J.; Goddard, A.; Clark, J. H.; Macquarrie, D. J. Chem. Commun. 2000, 46, 987.

[42] Danoun, G.; Bayarmagnai, B.; Gruenberg, M. F.; Goossen, L. J. Chem. Sci. 2014, 5, 1312.

[43] (a) Hu, M.; Rong, J.; Miao, W.; Ni, C.; Han, Y.; Hu, J.-B. Org. Lett. 2014, 16, 2030. (b) Wang, X.; Zhou, Y.; Ji, G.; Wu, G.; Li, M.; Zhang, Y.; Wang, J.-B. Eur. J. Org. Chem. 2014, 3093.

[44] Lefebvre, Q.; Fava, E.; Nikolaienko, P.; Rueping, M. Chem. Commun. 2014, 50, 6617.

[45] Matheis, C.; Krause, T.; Bragoni, V.; Goossen, L. J. Chem. Eur. J. 2016, 22, 12270 .

[46] Nikolaienko, P.; Pluta, R.; Rueping, M. Chem. Eur. J. 2014, 20, 9867.

[47] Liu, J.-B.; Xu, X.-H.; Chen, Z.-H.; Qing, F.-L. Angew. Chem. Int. Ed. 2015, 54, 897.

[48] Qiu, Y.-F.; Song, X.-R.; Li, M.; Zhu, X.-Y.; Wang, A.-Q.; Yang, F.; Han, Y.-P.; Zhang, H.-R.; Jin, D.-P.; Li, Y.-X.; Liang, Y.-M. Org. Lett. 2016, 18, 1514

[49] Ye, K.-Y.; Zhang, X.; Dai, L.-X.; You, S.-L. J. Org. Chem. 2014, $79,12106$.

[50] Zeng, J.-L.; Chachignon, H.; Ma, J.-A.; Cahard, D. Org. Lett. 2017, 19, 1974.

[51] Nikolaienko, P.; Yildiz, T.; Rueping, M. Eur. J. Org. Chem. 2016, 1091.

[52] Fang, W.-Y.; Dong, T.; Han, J.-B.; Zha, G.-F.; Zhang, C.-P. Org. Biomol. Chem. 2016, 14, 11502.

[53] Wang, K.-P.; Yun, S.-Y.; Mamidipalli, P.; Lee, D. Chem. Sci. 2013, 4, 3205.

[54] Karmakar, R.; Mamidipalli, P.; Salzman, R. M.; Hong, S.; Yun, S. -Y.; Guo, W.; Xia, Y.; Lee, D. Org. Lett. 2016, 18, 3530.

[55] Xiao, Q.; Sheng, J.; Ding, Q.; Wu, J. Eur. J. Org. Chem. 2014, 217.

[56] Zeng, Y. W.; Hu, J.-B. Org. Lett. 2016, 18, 856.

[57] Li, S.-G.; Zard, S. Z. Org. Lett. 2013, 15, 5898.

[58] Yang, H.-B.; Fan, X.; Wei, Y.; Shi, M. Org. Chem. Front. 2015, 2, 1088.

[59] Fan, X.; Yang, H.; Shi, M. Adv. Synth.Catal. 2017, 359, 49.

[60] (a) Andreades, S.; Harris, J. F.; Sheppard, W. A. J. Org. Chem. 1964, 29, 898. (b) Sheppard, W. A. J. Org. Chem. 1964, 29, 895; (c) Scribner, R. M. J. Org. Chem. 1966, 31, 3671. (d) Bayreuther, H.; Haas, A. Chem. Ber. 1973, 106, 1418. (e) Croft, T. S.; McBrady, J. J. J. Heterocycl. Chem. 1975, 12, 845. (f) Haas, A.; hellwig, V. Chem. Ber. 1976, 109, 2475. (g) Haas, A.; Niemann, U. Chem. Ber. 1977, 110, 67. (h) Popov, V. I.; Kondranenko, N. V.; Haas, A. UKr. Khim. Zh. 1983, 49, 861. (i) Haas, A.; Lieb, M.; Zhang, Y. J. Fluorine Chem. 1985, 29, 311; (j) Bogdanowicz-Szwed, K.; Kawalek, B.; Lieb, M. J. Fluorine Chem. 1987, 35, 317. (k) Rossman, D. I.; Muller, A. J.; Lewis, E. O. J. Fluorine Chem. 1991, 55, 221.

[61] (a) Sharpe, T. R.; Cherkofsky, S. C.; Hewes, W. E.; Smith, D. H.; Gregory, W. A.; Haber, S. B.; Leadbetter, M. R.; Whitney, J. G. J. Med. Chem. 1985, 28, 1188. (b) South, M. S.; Van Sant, K. A. J. Heterocycl. Chem 1991, 28, 1017. (c) Boese, R.; Haas, A.; Lieb, M.; Roeske, U. Chem. Ber. 1994, 127, 449.

[62] Tran, L. D.; Popov, I.; Daugulis, O. J. Am. Chem. Soc. 2012, 134, 18237.

[63] $\mathrm{CF}_{3} \mathrm{SCl}$ was reported to have an $\mathrm{L}(\mathrm{ct}) 50$ of between 440 and 880 $\mathrm{ppm} / \mathrm{min}$ and $\mathrm{CF}_{3} \mathrm{SSCF}_{3}$ was reported to have an $\mathrm{L}(\mathrm{ct}) 50$ of about
200 ppm/min. Chem. Eng. News 1967, 45(51), 44.

[64] Ferry, A.; Billard, T.; Langlois, B. R.; Bacque, E. J. Org. Chem. 2008, 73, 9362 .

[65] Ferry, A.; Billard, T.; Langlois, B. R.; Bacque, E. Angew. Chem. Int. Ed. 2009, 48, 8551.

[66] Alazet, S.; Zimmer, L.; Billard, T. Angew. Chem. Int. Ed. 2013, 52, 10814.

[67] Ferry, A.; Billard, T.; Bacqué, E.; Langlois, B. R. J. Fluorine Chem. 2012, 134, 160.

[68] Alazet, S.; Ollivier, K.; Billard, T. Beilstein J. Org. Chem. 2013, 9 , 2354

[69] (a) Alazet, S.; Zimmer, L.; Billard, T. Chem. Eur. J. 2014, 20, 8589. (b) Alazet, S.; Ismalaj, E.; Glenadel, Q.; Le Bars, D.; Billard, T. Eur. J. Org. Chem. 2015, 4607.

[70] Alazet, S.; Zimmer, L.; Billard, T. J. Fluorine Chem. 2015, 171, 78.

[71] Glenadel, Q.; Alazet, S.; Tlili, A.; Billard, T. Chem. Eur. J. 2015, 21, 14694.

[72] Glenadel, Q.; Billard, T. Chin. J. Chem. 2016, 34, 455.

[73] Glenadel, Q.; Bordy, M.; Alazet, S.; Tlili, A.; Billard, T. Asian J. Org. Chem. 2016, 5, 428.

[74] Tlili, A.; Alazet, S.; Glenadel, Q.; Billard, T. Chem. Eur. J. 2016, 22, 10230.

[75] Alazet, S.; Billard, T. Synlett 2015, 26, 76

[76] Bonazaba Milandou, L. J. C.; Carreyre, H.; Alazet, S.; Greco, G.; Martin-Mingot, A.; Nkounkou Loumpangou, C.; Ouamba, J. M.; Bouazza, F.; Billard, T.; Thibaudeau, S. Angew. Chem. Int. Ed. 2017, $56,169$.

[77] Yang, Y.; Jiang, X.; Qing, F.-L. J. Org. Chem. 2012, 77, 7538.

[78] (a) Liu, J.-B.; Chu, L.-L.; Qing, F.-L. Org. Lett. 2013, 15, 894. (b) Xu, J.-B.; Chen, P.-H.; Ye, J.-X.; Liu, G.-S. Acta Chim. Sinica 2015, 73, 1294 (in Chinese). (徐佳斌, 陈品红, 叶金星, 刘国生, 化学学 报, 2015, 73, 1294). (c) Wu, W.; Zhang, X.-X.; Liang, F.; Cao, S Org. Biomol. Chem. 2015, 13, 6992.

[79] Xiao, Q.; Sheng, J.; Chen, Z.; Wu, J. Chem. Commun. 2013, 49 , 8647.

[80] Sheng, J.; Fan, C.; Wu, J. Chem. Commun. 2014, 50, 5494

[81] Sheng, J.; Li, S.; Wu, J. Chem. Commun. 2014, 50, 578.

[82] Liu, T.; Qiu, G.-Y.-S.; Ding, Q.-P.; Wu, J. Tetrahedron 2016, 72, 1472.

[83] Sheng, J.; Wu, J. Org. Biomol. Chem. 2014, 12, 7629.

[84] Liu, Y.-W.; Qiu, G.-Y.-S.; Wang, H.-L.; Sheng, J. Tetrahedron Lett 2017, 58,690 .

[85] Shao, X.-X.; Wang, X.-Q.; Yang, T.; Lu, L.; Shen, Q.-L. Angew. Chem. Int. Ed. 2013, 52, 3457.

[86] Vinogradova, E. V.; Muller, P.; Buchwald, S. L. Angew. Chem. Int. Ed. 2014, 53, 3125 .

[87] Shao, X.-X.; Liu, T.-F.; Lu, L.; Shen, Q.-L. Org. Lett. 2014, 16, 4738.

[88] Ma, B.-Q.; Shao, X.-X.; Shen, Q.-L. J. Fluorine Chem. 2015, 171, 73.

[89] Shao, X.-X.; Liu, T.-F.; Lu, L.; Shen, Q.-L. Org. Lett. 2015, 80, 3012 .

[90] (a) Wang, X.-Q.; Yang, T.; Cheng, X.; Shen, Q.-L. Angew. Chem. Int. Ed. 2013, 52, 12860. (b) Yang, T.; Shen, Q.-L.; Lu, L. Chin. J. Chem. 2014, 32, 678 .

[91] Deng, Q. H.; Rettenmeier, C.; Wadepohl, H.; Gade, L. H. Chem. Eur. J. 2014, 20, 93

[92] He, H.; Zhu, X. Org. Lett. 2014, 16, 3102.

[93] Li, Y.; Ye, Z.; Bellman, T. M.; Chi, T.; Dai, M. Org. Lett. 2015, 17, 2186.

[94] Yang, Y.-D.; Azuma, A.; Tokunaga, E.; Yamasaki, M.; Shiro, M.; Shibata, N. J. Am. Chem. Soc. 2013, 135, 8782.

[95] Arimori, S.; Takada, M.; Shibata, N. Dalton Trans. 2015, 44, 19456.

[96] Huang, Z.; Yang, Y.-D.; Tokunaga, E.; Shibata, N. Asian J. Org. Chem. 2015, 4, 525.

[97] Huang, Z.; Yang, Y.-D.; Tokunaga, E.; Shibata, N. Org. Lett. 2015, 17,1094

[98] Arimori, S.; Takada, M.; Shibata, N. Org. Lett. 2015, 17, 1063.

[99] Huang, Z.; Okuyama, K.; Wang, C.; Tokunaga, E.; Li, X.; Shibata, N. ChemistryOpen 2016, 5, 188.

[100] Haas, A.; Möller, G. Chemische Berichte 1996, 129, 1383.

[101] Munavalli, S.; Rohrbaugh, D. K.; Rossman, D. I.; Berg, F. J.; Wagner, G. W.; Durst, H. D. Synth. Commun. 2000, 30, 2847.

[102] Pluta, R.; Nikolaienko, P.; Rueping, M. Angew. Chem. Int. Ed. 2014, 53,1650 . 
[103] Kang, K.; Xu, C.-F.; Shen, Q.-L. Org. Chem. Front. 2014, 1, 294.

[104] Bootwicha, T.; Liu, X.; Pluta, R.; Atodiresei, I.; Rueping, M. Angew. Chem. Int. Ed. 2013, 52, 12856.

[105] Rueping, M.; Liu, X.; Bootwicha, T.; Pluta, R.; Merkens, C. Chem. Commun. 2014, 50, 2508.

[106] Pluta, R.; Rueping, M. Chem. Eur. J. 2014, 20, 17315.

[107] Xiao, Q.; He, Q.; Li, J.; Wang, J. Org. Lett. 2015, 17, 6090.

[108] Honeker, R.; Ernst, J. B.; Glorius, F. Chem. Eur. J. 2015, 21, 8047.

[109] Xu, C.-F.; Shen, Q.-L. Org. Lett. 2014, 16, 2046.

[110] Zhao, B.-L.; Du, D.-M. Org. Lett. 2017, 19, 1036

[111] Xu, C.--F.; Ma, B.-Q.; Shen, Q.-L. Angew. Chem. Int. Ed. 2014, 53, 9316.

[112] Xu, C.-F.; Shen, Q.-L. Org. Lett. 2015, 17, 4561.

[113] Wang, Q.; Qi, Z.; Xie, F.; Li, X.-W. Adv. Synth. Catal. 2015, 357, 355.

[114] Wang, Q.; Xie, F.; Li, X.-W. J. Org. Chem. 2015, 80, 8361.

[115] Maeno, M.; Shibata, N.; Cahard, D. Org. Lett. 2015, 17, 1990.

[116] Luo, J.; Zhu, Z.; Liu, Y.; Zhao, X.-D. Org. Lett. 2015, 17, 3620.

[117] Wu, J.-J.; Xu, J.; Zhao, X.-D. Chem. Eur. J. 2016, 22, 15265.

[118] Liu, X.; An, R.; Zhang, X.; Luo, J.; Zhao, X.-D. Angew. Chem. Int. Ed. 2016, 55, 5846.

[119] Yu, Y.; Xiong, D.-C.; Ye, X.-S. Org. Biomol. Chem. 2016, 14, 6403.

[120] Hu, L.-Q.; Wu, M.-H.; Wan, H.-X.; Wang, J.; Wang, G.-Q.; Guo, H.-B.; Sun, S.-F. New J. Chem. 2016, 40, 6550.

[121] Ernst, J. B.; Rakers, L.; Glorius, F. Synthesis 2017, 49, 260.

[122] Wei, F.; Zhou, T.; Ma, Y.; Tung, C.-H.; Xu, Z.-H. Org. Lett. 2017, 19, 2098.

[123] Zhang, P.-P.; Li, M.; Xue, X.-S.; Xu, C.-F.; Zhao, Q.-C.; Liu, Y.-F.; Wang, H.-Y.; Guo, Y.-L.; Lu, L.; Shen, Q.-L. J. Org. Chem. 2016, 81,7486 .

[124] Li, M.; Guo, J.; Xue, X.-S.; Cheng, J.-P. Org. Lett. 2016, 18, 264.

[125] Zhang, H.; Leng, X.-B.; Wan, X.-L.; Shen, Q.-L. Org. Chem. Front. 2017, 4, 1051.

[126] Zhu, X.-L.; Xu, J.-H.; Cheng, D.-J.; Zhao, L.-J.; Liu, X.-Y.; Tan, B. Org. Lett. 2014, 16, 2192.

[127] Xiang, H.; Yang, C.-H. Org. Lett. 2014, 16, 5686.

[128] Zhu, S.-Q.; Xu, X.-H.; Qing, F.-L. Eur J. Org. Chem. 2014, 4453.

[129] Jiang, L.; Qian, J.; Yi, W.; Lu, G.; Cai, C.; Zhang, W. Angew. Chem. Int. Ed. 2015, 54, 14965 .

[130] Chachignon, H.; Maeno, M.; Kondo, H.; Shibata, N.; Cahard, D. Org. Lett. 2016, 18, 2467.

[131] Lu, K.; Deng, Z.-J.; Li, M.; Li, T.-J.; Zhao, X. Org. Biomol. Chem. 2017, 15, 1254.

[132] Jiang, L.-Q.; Yi, W.-B.; Liu, Q.-R. Adv. Synth. Catal. 2016, 358, 3700.
[133] Bu, M. J.; Lu, G. P.; Cai, C. Org. Chem. Front. 2017, 4, 266.

[134] Saidalimu, I.; Suzuki, S.; Tokunaga, E.; Shibata, N. Chem. Sci. 2016, 7, 2106.

[135] Saidalimu, I.; Suzuki, S.; Yoshioka, T.; Tokunaga, E.; Shibata, N. Org. Lett. 2016, 18, 6404.

[136] Harris, J. F.; Stacey, F. W. J. Am. Chem. Soc. 1961, 83, 840.

[137] (a) Harris, J. F. J. Am. Chem. Soc. 1962, 84, 3148. (b) Harris, J. F. J. Org. Chem. 1966, 31, 931.

[138] Haran, G.; Sharp, D. W. A. J. Chem. Soc., Perkin Trans. 1 1972, 34.

[139] Yin, F.; Wang, X.-S. Org. Lett. 2014, 16, 1128.

[140] Zhang, K.; Liu, J.-B.; Qing, F.-L. Chem. Commun. 2014, 50, 14157.

[141] Li, C.; Zhang, K.; Xu, X.-H.; Qing, F.-L. Tetrahedron Lett. 2015, 56, 6273.

[142] Fuentes, N.; Kong, W.; Fernandez-Sanchez, L.; Merino, E.; Nevado, C. J. Am. Chem. Soc. 2015, 137, 964.

[143] Qiu, Y.-F.; Zhu, X.-Y.; Li, Y.-X.; He, Y.-T.; Yang, F.; Wang, J.; Hua, H.-L.; Zheng, L.; Wang, L.-C.; Liu, X.-Y.; Liang, Y.-M. Org. Lett. 2015, 17,3694

[144] Jin, D.-P.; Gao, P.; Chen, D.-Q.; Chen, S.; Wang, J.; Liu, X.-Y.; Liang, Y.-M. Org. Lett. 2016, 18, 3486.

[145] Zeng, Y.-F.; Tan, D.-H.; Chen, Y.; Lu, W.-X.; Liu, X.-G.; Li, Q.; Wang, H.-G. Org. Chem. Front. 2015, 2, 1511.

[146] Pan, S.; Huang, Y.; Qing, F.-L. Chem. Asian J. 2016, 11, 2854.

[147] Wu, W.; Dai, W.; Ji, X.; Cao, S. Org. Lett. 2016, 18, 2918.

[148] Chen, M.-T.; Tang, X.-Y.; Shi, M. Org. Chem. Front. 2017, 4, 86.

[149] Li, M.; Petersen, J. L.; Hoover, J. M. Org. Lett. 2017, 19, 638.

[150] Liu, K.; Jin, Q.; Chen, S.; Liu, P.-N. RSC Adv. 2017, 7, 1546.

[151] Honeker, R.; Garza-Sanchez, R. A.; Hopkinson, M. N.; Glorius, F. Chem. Eur. J. 2016, 22, 4395.

[152] Li, Y.; Koike, T.; Akita, M. Asian J. Org. Chem. 2017, 6, 445.

[153] Dagousset, G.; Simon, C.; Anselmi, E.; Tuccio, B.; Billard, T.; Magnier, E. Chem. Eur. J. 2017, 23, 4282.

[154] Hu, F.; Shao, X.-X.; Zhu, D.-H.; Lu, L.; Shen, Q.-L. Angew. Chem. Int. Ed. 2014, 53, 6105

[155] Yang, T.; Lu, L.; Shen, Q.-L. Chem. Commun. 2015, 51, 5479.

[156] Candish, L.; Pitzer, L.; Gomez-Suarez, A.; Glorius, F. Chem. Eur. J. 2016, 22, 4753.

[157] He, B.; Xiao, Z.-W.; Wu, H.; Guo, Y.; Chen, Q.-Y.; Liu, C. RSC $A d v$. 2017, 7, 880

[158] (a) Wu, H.; Xiao, Z.; Wu, J.; Guo, Y.; Xiao, J.-C.; Liu, C.; Chen, Q.-Y. Angew. Chem. Int. Ed. 2015, 54, 4070. (b) Guo, S.; Zhang, X.; Tang, P.-P. Angew. Chem. Int. Ed. 2015, 54, 4065.

[159] Mukherjee, S.; Maji, B.; Tlahuext-Aca, A.; Glorius, F. J. Am. Chem. Soc. 2016, 138, 16200.

[160] Li, H.; Shan, C.; Tung, C.-H.; Xu, Z.-H. Chem. Sci. 2017, 8, 2610.

(Cheng, B.) 